\title{
Manuel pour la préparation, la mise en œuvre, le suivi, le reporting et l'évaluation de la réforme de l'administration publique et des stratégies sectorielles
}

\author{
Conseils pour les partenaires de SIGMA
}

\section{DOCUMENT SIGMA No. 57}

Publication autorisée par Karen Hill, Directrice du Programme SIGMA

Ce document est produit avec le soutien financier de l'Union européenne (UE). Il ne doit pas être présenté comme exprimant les vues officielles de l'UE, de l'OCDE ou de ses pays membres, ou des pays partenaires participant au Programme SIGMA. Les opinions exprimées et les arguments employés sont ceux des auteurs. 


\section{Table des matières}

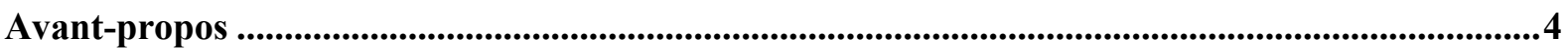

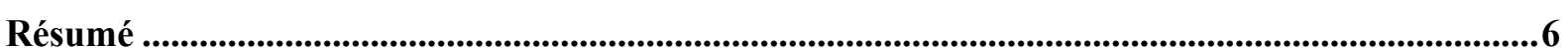

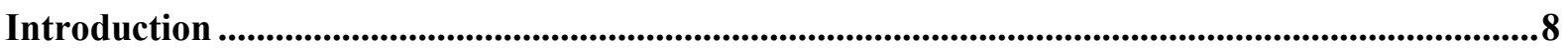

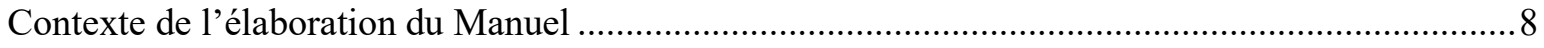

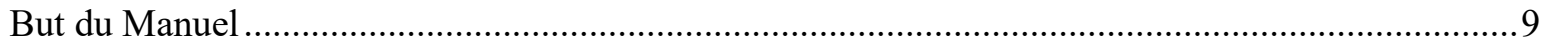

Quelle est la destination de ce Manuel ? ..................................................................................

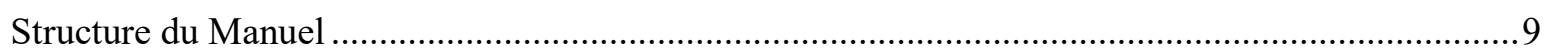

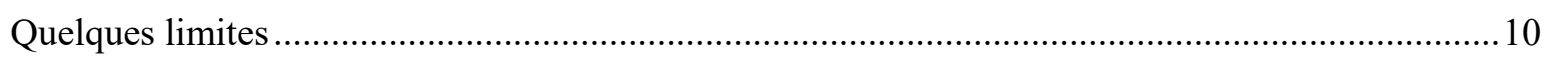

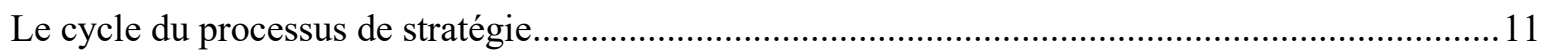

Pourquoi la planification préalable est-elle importante pour l'élaboration d'une stratégie ?............14

Questions clés pour la planification du processus d'élaboration de stratégie..................................15

1. Analyse de problème ....................................................................................................................................17

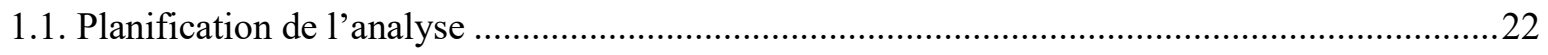

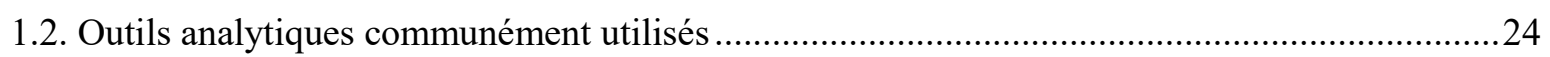

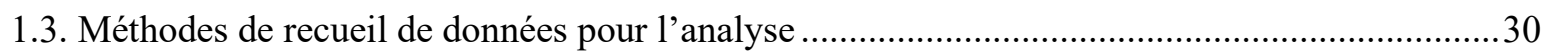

1.4. Comment définir le cercle des institutions impliquées pour l'analyse .......................................31

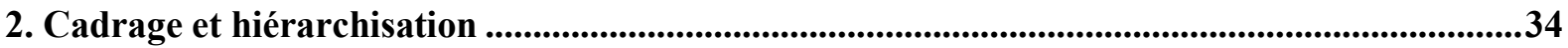

2.1. Outil de priorisation SIGMA pour l'élaboration d'une stratégie de RAP ..................................35

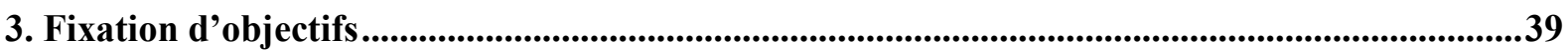

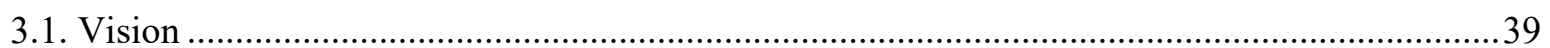

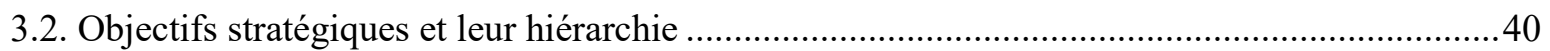

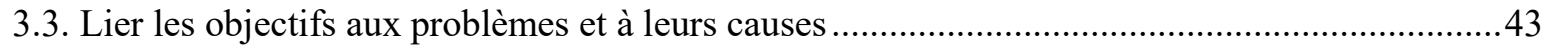

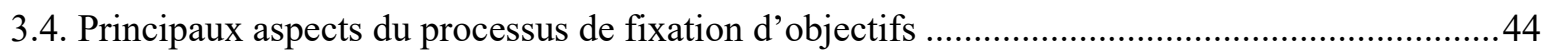

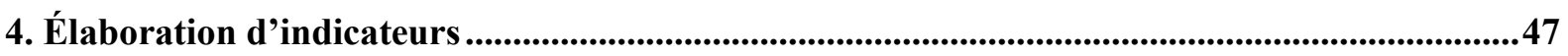

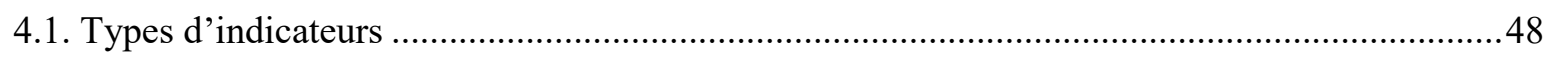

4.2. Les liens entre objectifs et actions et les indicateurs mesurant leur réalisation...........................51

4.3. Désignation des personnes chargées de la mesure et de la qualité des indicateurs .....................52

4.4. Établir des valeurs de référence et des valeurs cibles...............................................................54

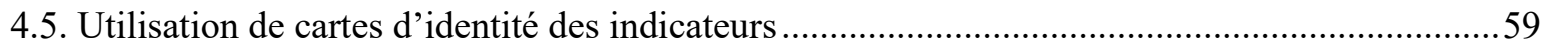

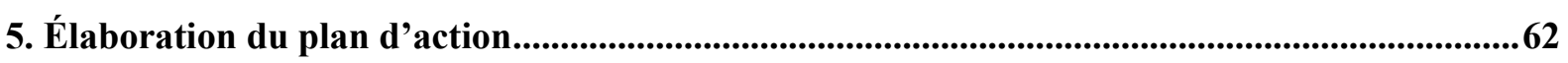

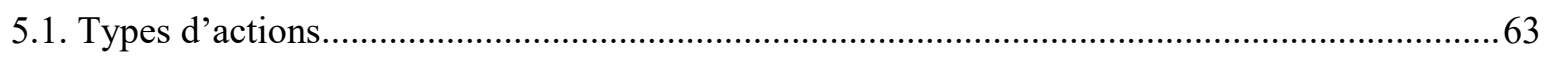

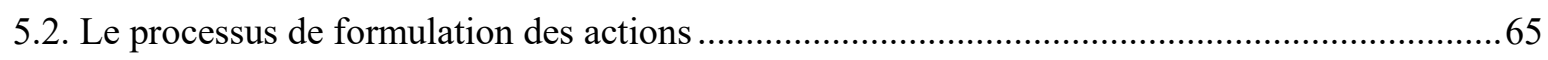




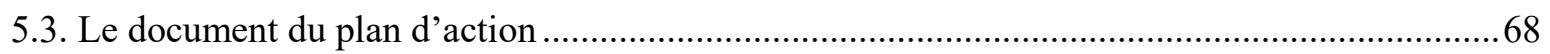

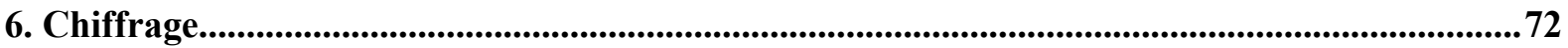

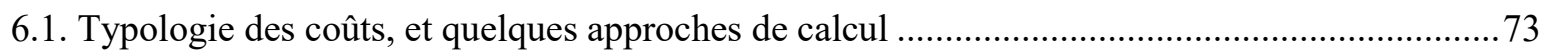

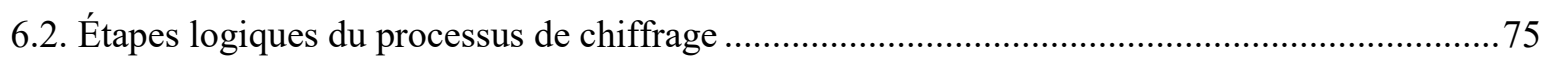

6.3. Répercussion des résultats du chiffrage avant la finalisation de l'élaboration de la stratégie....79

7. Suivi et reporting (rédaction de rapports) ........................................................................82

7.1. Le rôle du suivi, du reporting et de l'évaluation dans le processus stratégique ..........................84

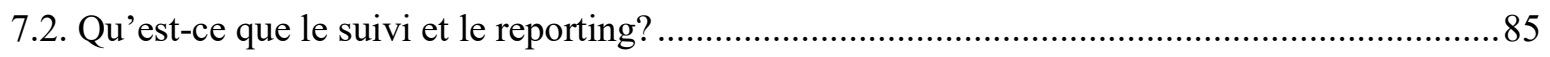

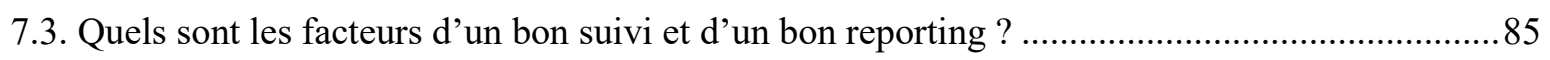

7.4. Organisation et rôles institutionnels pour le suivi et l'élaboration de rapports de stratégie.......90

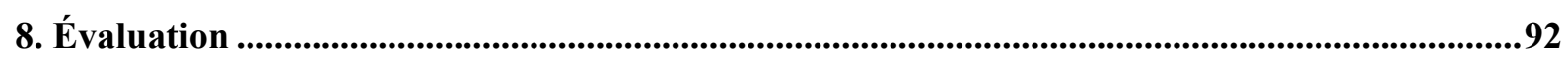

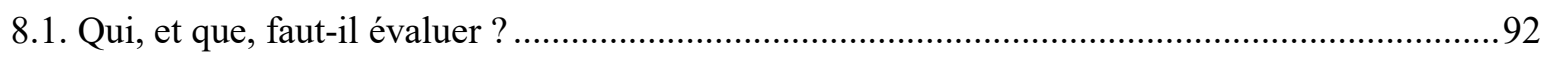

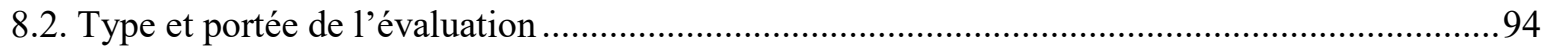

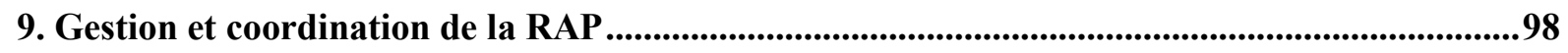

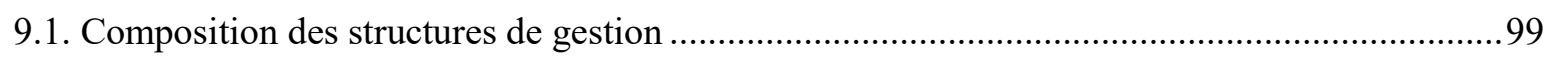

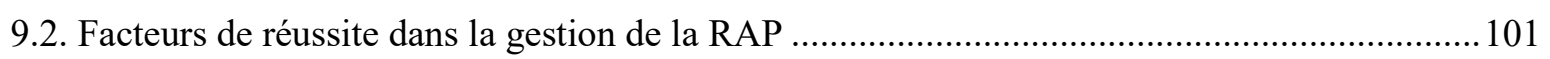

Glossaire de certains termes clés utilisés dans le Manuel ....................................................................106

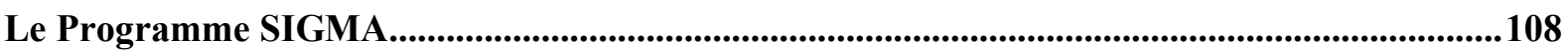




\section{Avant-propos}

Une administration publique qui fonctionne bien est une condition essentielle pour une gouvernance démocratique transparente et efficace. Elle constitue la base du fonctionnement de l'État, et détermine la capacité d'un gouvernement à fournir des services publics et à promouvoir la compétitivité et la croissance.

Atteindre les indispensables normes d'administration publique nécessite des réformes dans de nombreux domaines des politiques publiques et de l'administration. Si ces réformes sont conçues et mises en œuvre de façon fragmentée et au coup par coup, elles peuvent ne pas transformer le système et le fonctionnement d'une administration publique de la manière qui était prévue ${ }^{1}$. Pour atteindre des résultats, les gouvernements doivent piloter et coordonner la mise en œuvre d'une vision globale de la réforme et d'objectifs priorisés.

Un leadership clair et efficace, des mécanismes de mise en œuvre performants, des règles de responsabilité claires et la viabilité financière sont au cœur de toute stratégie de réforme réussie. Ces éléments sont critiques pour garantir qu'une stratégie est effectivement mise en œuvre, et n'existe pas que sur le papier.

Au cours des années, SIGMA a apporté son assistance à de nombreux pays pour le développement de plans de réforme de leur administration publique, ainsi que pour l'élaboration et la mise en place des cadres de surveillance et d'établissement de rapports correspondant à ces réformes. SIGMA a également aidé les pays à développer leurs systèmes nationaux de planification stratégique, pour garantir que la même qualité de normes et d'approches conceptuelles était appliquée à leur stratégie de développement et de suivi par secteur, indépendamment des spécificités du domaine de politique publique visé.

Ce Manuel fournit des informations et conseils pratiques, ainsi que des outils faciles à utiliser pour les personnes impliquées dans l'élaboration et la mise en œuvre de la réforme de l'administration publique (RAP) et des stratégies sectorielles. Il se fonde sur les leçons apprises, recommandations, conseils et outils développés et promus par SIGMA et par le reste de la Direction de la Gouvernance publique de l'OCDE.

Ce Manuel guide le lecteur à travers chaque étape du développement, de la mise en œuvre, du suivi et de la gestion générale des stratégies. Il couvre :

- l'analyse de problèmes ;

- la hiérarchisation des ambitions de réforme ;

- la fixation d'objectifs ;

- la définition d'indicateurs (avec références et cibles);

- la planification et le chiffrage des actions ;

- la mise en œuvre, le suivi, la réalisation de rapports et l'évaluation ;

- la gestion et l'apprentissage de la RAP.

\footnotetext{
${ }^{1}$ Pour une meilleure compréhension des différences entre les notions de changement et de réforme, voir : Huerta Melchor, O. (2008), « La gestion du changement dans l'administration des pays de l'OCDE : Un premier aperçu général », Documents de travail sur la gouvernance publique de l'OCDE, No. 12, Éditions OCDE, Paris, https://dx.doi.org/10.1787/227138426766.
} 
Il fournit également des nombreux outils, recommandations et modèles.

L'élaboration de ce Manuel a été dirigée par Péter Vági du Programme SIGMA et Egle Rimkute de Lituanie, avec la contribution spécialisée significative de Mārtiņš Krieviņš de Lettonie. L'équipe tient également à remercier Dragan Djuric, Zuhra OsmanovićPašić, Jolanda Trebicka, Milena Lazarević, Ljiljana Uzelac et Ruzhdi Halili qui ont apporté leurs conseils et leur expertise, ainsi que les collègues de la Commission européenne (CE) et de la Direction de la Gouvernance publique de l'OCDE qui ont révisé le texte, y ont apporté des commentaires et suggestions extrêmement précieux. 


\section{Résumé}

Au cours des dernières décennies, le fonctionnement de l'administration publique est devenu une composante clé des débats sur le développement économique et la démocratisation.

La reconnaissance de l'importance primordiale d'une administration publique responsable pour la gouvernance démocratique et le développement économique est cohérente avec les Objectifs de développement durable des Nations unies ${ }^{2}$. L'Objectif 16 - parmi d'autres - renvoie à la promotion de sociétés pacifiques et inclusives, à l'ouverture de l'accès à la justice pour tous et à la mise en place d'institutions efficaces et responsables à tous les niveaux. Par ailleurs, l'Objectif 8 renvoie à la promotion d'une croissance économique durable, de l'emploi et du travail décent pour tous.

Une administration publique performante présente des avantages et des bénéfices pour les individus ainsi que pour l'État. En premier lieu, elle permet aux gouvernements de réaliser leurs objectifs de politique publique et d'assurer la mise en œuvre adéquate des décisions politiques et règles juridiques, et assure en conséquence l'efficacité et la stabilité politique. À l'inverse, une administration publique défaillante occasionne des retards, de l'inefficacité, de l'incertitude, de la corruption et d'autres formes de mauvaise gestion qui nourrissent l'exaspération et le désenchantement des citoyens, et les incitent à résister à l'État et à ses institutions et à les contester. La légitimité du gouvernement est ainsi sapée, ce qui peut conduire à la défaillance de l'État.

En second lieu, l'importance de l'administration publique pour le développement de l'économie est internationalement reconnue ${ }^{3}$. Avec une législation appropriée et un système judiciaire indépendant et efficace, une bonne administration publique est un fondement essentiel d'un marché performant. Pour évaluer les risques, les investisseurs s'appuient largement sur la prédictibilité des décisions administratives, qui dépend de la stabilité de l'environnement politique et institutionnel. La mauvaise gestion, sous la forme de défaillances administratives et de processus administratifs longs et inutilement complexes, entrave les initiatives économiques de la part des investisseurs potentiels, nationaux ou étrangers, et a donc des effets négatifs sur l'emploi et la stabilité politique ${ }^{4}$.

L'un des aspects importants d'une administration publique efficace réside en la capacité de planifier d'importantes réformes, de manière à ce que des objectifs politiques bien

2 Nations unies (2015), Objectifs de développement durable, https://www.un.org/sustainabledevelopment/fr/objectifs-de-developpement-durable/.

3 OCDE (2017), Panorama des administrations publiques 2017, Éditions OCDE, Paris, https://doi.org/10.1787/gov_glance-2017-fr. Voir aussi : World Public Sector Report 2015 du Département des affaires économiques et sociales des Nations unies, http://workspace.unpan.org/sites/Internet/Documents/UNPAN95253.pdf.

${ }^{4}$ Prenant acte de ces défis et de la nécessité d'intégrer des connaissances comparatives et des bonnes pratiques internationales, la Direction de la Gouvernance publique de l'OCDE développe actuellement un Cadre stratégique sur la bonne gouvernance publique, qui vise à être un outil de diagnostic flexible pour aider les gouvernements à élaborer et mettre en œuvre des approches efficaces pour les réformes de la gouvernance publique. 
définis soient poursuivis à travers des actions ciblées, conformément aux ressources disponibles et d'une manière logique. Ces actions devraient cibler le cœur des lacunes ou problèmes identifiés auxquels les réformes tentent de remédier.

Les documents de planification stratégique et opérationnelle doivent fournir une feuille de route claire pour la mise en œuvre des politiques individuelles. Ces documents de planification doivent traduire les déclarations de priorité au niveau politique en objectifs et cibles de réforme, identifier les actions à entreprendre et les institutions responsables pour le faire, y affecter les ressources nécessaires et fournir les autres informations pertinentes pour la mise en œuvre du programme de réforme. Une fois que les documents de planification sont en place, la mise en œuvre doit être soutenue par un financement adéquat ainsi que par des moyens administratifs dans les principales institutions impliquées.

Étant donné que les réformes exigent des ressources substantielles et une mise en œuvre ciblée sur plusieurs années, il est également crucial d'assurer la création et le fonctionnement d'un système de gestion et de coordination adéquat, système permettant des actions correctives, si nécessaire, afin de garantir une mise en œuvre réussie. De tels systèmes ne peuvent fonctionner efficacement que s'ils opèrent sur la base de données solides concernant les progrès de la mise en œuvre et l'avancement des réformes par rapport aux objectifs fixés. C'est pourquoi le développement d'un sérieux système de suivi et de réalisation de rapports ainsi qu'une évaluation des résultats de la réforme sont nécessaires pour que les décideurs puissent efficacement piloter les processus de réforme.

Le Manuel pour la préparation, la mise en œuvre, le suivi, la réalisation de rapports et l'évaluation de la RAP et des stratégies sectorielles constitue une synthèse des leçons apprises et des outils développés pendant plus de 25 ans par SIGMA, à travers son travail sur ces questions avec ses partenaires.

L'audience cible principale de ce Manuel comprend le personnel des institutions de l'administration centrale, des principaux ministères, agences et autres institutions du secteur public qui ont pour mission de préparer et de mettre en œuvre des stratégies et leurs plans d'action. Ce Manuel peut de surcroît représenter une source utile pour les parties prenantes de la coopération pour le développement tels que les partenaires du développement, les organisations de mise en œuvre impliquées dans l'aide au développement, et les organisations non gouvernementales et de la société civile travaillant dans les divers domaines de l'administration publique. 


\section{Introduction}

\section{Contexte de l'élaboration du Manuel}

En 2014, la CE a introduit la RAP comme troisième pilier de la politique d'élargissement, en même temps que l'État de droit et la gouvernance économique. La Commission a, pour la première fois, donné sa définition de la RAP, soulignant que celle-ci recouvrait les dimensions essentielles suivantes : cadre stratégique de la RAP, élaboration et coordination des politiques, gestion des services publics et des ressources humaines, reddition de comptes, prestation de services et gestion des finances publiques. La RAP est maintenant considérée comme faisant partie des réformes clés que les pays doivent engager dès le début du processus d'adhésion, comme condition préalable pour un processus d'adhésion réussi et pour, à terme, un fonctionnement efficace en tant que pays membre.

L'importance de la RAP a également été soulignée dans le réexamen de la Politique européenne de voisinage (PEV) en 2015 et dans la Stratégie globale de l'UE en 2016. Ces documents stratégiques, qui donnent la même définition de la RAP que celle utilisée dans le contexte de l'élargissement, insistent spécialement sur l'importance d'avoir une administration publique responsable, celle-ci étant cruciale pour la gouvernance démocratique et le développement économique.

SIGMA, à la demande de la CE, a élaboré les Principes d'administration publique ${ }^{5}$ (les Principes) pour définir les conditions d'une administration publique performante telle qu'elle est préconisée dans la politique d'élargissement et la PEV. Ces Principes mettent en œuvre les principes universels de bonne gouvernance, notamment ceux qui sont préconisés par les Objectifs de développement durable, en particulier l'Objectif $16^{6}$.

Les Principes établissent le cadre conceptuel, les conditions de base et les principaux aspects d'une administration publique efficace et efficiente. Ils fournissent également un cadre structuré à l'intérieur duquel les systèmes et méthodes des administrations publiques des pays candidats à l'adhésion à l'UE et des pays de la PEV peuvent être analysés et des recommandations peuvent être faites.

Dans le cadre de son approche renforcée de la RAP, la CE recommande également que certains Principes pertinents d'administration publique soient intégrés (généralisés) dans le travail sectoriel ${ }^{7}$ tant par les gouvernements que par la $\mathrm{CE}$, afin de garantir que les efforts horizontaux de réforme de l'administration publique ne sont pas compromis par des approches contradictoires des différents secteurs.

5 OCDE (2017), SIGMA, Principes d'administration publique, Éditions OCDE, Paris, http://www.sigmaweb.org/publications/Principles-Public-Administration-Fr.pdf et OCDE (2016), SIGMA, Principes d'administration publique: Un cadre pour les pays $d u$ voisinage européen, OCDE, Paris, http://www.sigmaweb.org/publications/Principles-ENP-\%200verviewFr.pdf.

${ }^{6}$ En particulier ses cibles $16.6,16.7,16.10$ et 16.A.

${ }^{7}$ L'intégration des Principes d'administration publique dans le travail par secteur est reflétée dans certains documents récents de la $\mathrm{CE}$ tels que le Manuel de jumelage: https://www.bmwi.de/Redaktion/DE/Downloads/S-T/twinning-manuel-commun-de-jumelage2017.pdf? blob $=$ publicationFile $\& \mathrm{v}=8$ 


\section{But du Manuel}

Ce Manuel pour la préparation, la mise en œuvre, le suivi, la réalisation de rapports et l'évaluation de la RAP et des stratégies sectorielles a pour but d'aider les gouvernements et administrations publiques déterminés à atteindre les cibles de l'Objectif de développement durable 16 et les Principes.

Le Manuel est directement lié aux Principes dans les domaines du cadre stratégique de la RAP et de l'élaboration et de la coordination des politiques.

La RAP et l'élaboration et la mise en ouvre de stratégies sectorielles sont des processus qui exigent d'identifier les principaux problèmes rencontrés par l'administration d'un pays donné, d'établir des objectifs et cibles clairs pour résoudre ces problèmes, et de traduire ces cibles et objectifs en actions spécifiques. Le processus d'élaboration de stratégies de RAP diffère de celui de développement de stratégies sectorielles en ce qu'il traite de sujets qui sont transversaux par nature et qui affectent le fonctionnement de l'administration publique dans son ensemble (par exemple la qualité de la gestion des ressources humaines, la responsabilité des institutions du secteur public, une saine gestion des finances publiques et une prise de décisions fondée sur des données factuelles). En conséquence, la gestion de la RAP requiert des formes de gestion spécifiques conçues selon cet aspect transversal, qui sont donc différente de la gestion de stratégies spécifiques à des secteurs.

\section{Quelle est la destination de ce Manuel ?}

Ce Manuel fournit des informations et conseils pratiques, ainsi que des outils d'utilisation facile pour les personnes impliquées dans l'élaboration et la mise en œuvre de la RAP et des stratégies sectorielles. Par conséquent, le public cible principal du Manuel est les personnels de l'administration centrale, des principaux ministères, agences et autres institutions du secteur public qui ont pour mission de préparer et de mettre en œuvre des stratégies et leurs plans d'action. Ce Manuel peut aussi représenter une source utile pour les parties prenantes de la coopération pour le développement, tels les partenaires de développement, les organisations chargées de la mise en œuvre de l'aide au développement, et les organisations non gouvernementales et de la société civile travaillant dans les divers domaines de l'administration publique.

Le Manuel est conçu de telle façon qu'il puisse être appliqué dans les régions concernées par l'élargissement de l'UE et du Voisinage européen quand il devient clair que les réformes horizontales et sectorielles complexes ne produisent pas les résultats escomptés et n'auront pas l'impact désiré si elles sont planifiées et mises en œuvre de manière fragmentée et au coup par coup.

\section{Structure du Manuel}

Dans les chapitres suivants, le Manuel guidera le lecteur à travers chaque étape de la préparation, de la mise en œuvre, du suivi et de la gestion globale des stratégies. Il couvre l'analyse de problèmes, la priorisation des ambitions de réforme, la fixation d'objectifs, la définition d'indicateurs (avec références et cibles), la planification et le chiffrage des actions, la mise en œuvre, le suivi, la réalisation de rapports et l'évaluation, et, pour la RAP, la gestion et l'apprentissage du processus. Le Manuel (avec ses Annexes) propose de nombreuses recommandations, d'outils faciles à utiliser, et de modèles pour aider à élaborer et mettre en œuvre des stratégies. 
Le Manuel est structuré selon une approche par cycle/phases, montrant et expliquant les interconnexions inhérentes aux différentes étapes de l'élaboration et de la mise en œuvre d'une stratégie. Par exemple, la pertinence des objectifs d'une stratégie dépend de la manière dont les problèmes ont été identifiés et, en retour, la clarté des objectifs de réforme influence le succès avec lequel vont être fixés les indicateurs pertinents pour leur mesure. L'approche cyclique révèle également à quel point l'élaboration et la mise en œuvre de stratégies ne sont pas des exercices ponctuels, mais plutôt un processus continuel d'apprentissage, permettant la révision des objectifs et des actions stratégiques sur la base des leçons apprises. Cette approche aide également à expliquer les processus d'élaboration et de gestion de stratégies spécifiques à la $\mathrm{RAP}^{8}$.

Le Manuel est constitué de :

- Un document explicatif (chapitres suivants) détaillant le cycle d'élaboration et de mise en œuvre d'une stratégie, fournissant des exemples liés à la RAP venant d'expériences du travail de SIGMA avec ses partenaires et développés plus avant pour ce Manuel, avec des références à des documents additionnels élaborés par d'autres institutions ;

- Des Annexes contenant des outils de soutien (modèles et cadres) pour une utilisation ultérieure par les lecteurs, lorsqu'ils auront à arrêter et mettre en œuvre des stratégies, ainsi que des exemples détaillés additionnels présentés à titre d'illustration.

\section{Quelques limites}

Bien que ce Manuel serve de guide utile pour l'élaboration de stratégies dans tout secteur particulier, les personnes chargées de l'élaboration d'une stratégie sectorielle doivent s'assurer que la stratégie respecte les règles prévues au niveau de l'administration centrale pour l'élaboration des stratégies sectorielles. En outre, dans la mesure où les structures de gestion pour l'élaboration et la mise en œuvre de stratégies sectorielles dépendent de chaque secteur et de son contexte, ce Manuel ne fournit pas de conseils sur la manière dont la gestion de stratégies sectorielles devrait être menée ; il traite de la gestion stratégique et de la coordination de la RAP. Par conséquent, alors que la même logique et que les rôles et responsabilités clés des principaux forums de coordination décrits sont applicables à tous les secteurs, certains aspects ne s'appliquent aux stratégies de RAP seulement, étant donné la nature transversale de celle-ci.

SIGMA n'est en rien responsable du contenu de documents développés par d'autres organisations et mentionnés dans ce Manuel. Les auteurs ont toutefois jugé que ces documents étaient potentiellement intéressants pour les lecteurs désirant approfondir leurs connaissances au-delà des domaines traités par le Manuel.

La terminologie utilisée dans ce Manuel n'est pas nécessairement la même que celle utilisée dans les documents référencés. Les termes, phrases et concepts expliqués dans

\footnotetext{
${ }^{8}$ Dans la même lignée que l'approche par cycle utilisée dans ce Manuel, les Lignes directrices de la DG NEAR et d'autres documents liés à l'élaboration de stratégies utilisent l'approche dite d'«intervention logique» pour illustrer le chemin séquentiel et temporel de la définition d'interventions stratégiques. Pour plus d'informations à ce sujet, voir Commission européenne (2016), DG NEAR Guidelines on linking planning/programming, monitoring and evaluation, Bruxelles, p. 30 (https://ec.europa.eu/neighbourhood-enlargement/sites/near/files/ pdf/financial_assistance/phare/evaluation/2016/20160831-dg-near-guidelines-on-linkingplanning-progrming-vol-1-v0.4.pdf).
} 
le Manuel sont uniquement basés sur l'expérience et la pratique de SIGMA. Par exemple, bien que le Manuel vise à s'harmoniser avec les Lignes directrices de 2016 de la DG NEAR sur les liens entre la planification, la programmation, le suivi et l'évaluation, certaines différences terminologiques peuvent exister entre les deux documents.

Les modèles inclus dans les Annexes ont été élaborés par SIGMA de manière à ce que tout utilisateur puisse les adapter et les compléter selon ses besoins particuliers. SIGMA ne peut en aucun cas assumer la responsabilité du contenu développé lors de l'utilisation des modèles.

Ce document n'est pas conçu pour être lu du début jusqu'à la fin. Il doit plutôt être considéré comme un guide pouvant être utile aux utilisateurs durant les différentes étapes de l'élaboration et de la mise en œuvre de stratégies. Pour cette raison, nous suggérons que le lecteur utilise les différentes parties du Manuel lorsqu'elles sont pertinentes dans son processus effectif de travail.

L'élaboration de ce Manuel et de ses Annexes est fondée sur l'expérience que SIGMA a accumulée durant des années de travail avec ses partenaires, et sur la synthèse de bonnes pratiques et d'idées conceptuelles internationales concernant le développement et la mise en œuvre de stratégies. En tant que tel, ce document est un aperçu de conseils fondés sur les connaissances et l'expérience actuelles. L'élaboration et la mise en œuvre de stratégies étant un sujet majeur de la littérature internationale, ainsi qu'un domaine clé du travail de SIGMA, ce Manuel doit être considéré comme un document vivant qui pourra être réexaminé et révisé sur la base de nouvelles expériences et développements dans le contexte international.

\section{Le cycle du processus de stratégie}

La préparation, la mise en œuvre, le suivi, la réalisation de rapports et l'évaluation de stratégies représentent un processus dynamique, complexe, itératif et interactif par lequel le gouvernement - au cours d'un processus inclusif qui comprend des parties prenantes internes et externes - identifie les problèmes, définit et hiérarchise ses objectifs, planifie les actions pour atteindre ces objectifs (en en calculant les coûts et en en identifiant les sources de financement), et définit un cadre de mesure (comprenant des cibles) pour valider les progrès et aider à résoudre les difficultés lors de la phase de mise en œuvre. En tant que tel, le processus stratégique suit généralement un cycle d'élaboration des politiques universellement conçu et appliqué, dont les étapes sont les suivantes :

1. L'analyse des problèmes - Analyse de l'état actuel de la situation (réalisations, défis et opportunités), de préférence également fondée sur les leçons apprises du suivi et des rapports d'évaluation de précédentes réformes, quand ils existent ;

2. La hierarchisation des problèmes et des objectifs - Examen et sélection des problèmes qui doivent être résolus, sur la base de leur caractère d'urgence, de leur importance ou de leur ampleur, conformément aux priorités du gouvernement et aux ressources disponibles, et à la lumière des avis des institutions concernées et, plus largement, de l'ensemble des parties prenantes ;

3. Fixation d'objectifs - Définition du niveau d'ambition pour le changement par rapport à l'état actuel de la situation, par rapport aux problèmes dont la résolution a été jugée prioritaire ;

4. Définition d'indicateurs avec des valeurs de référence, des étapes et des cibles - Élaboration de manières de mesurer la réalisation des objectifs définis, ainsi 
que fixation de cibles mesurables qui mesurent le degré et la direction du changement envisagé ainsi que le niveau de performance attendu ;

5. Planification d'actions - Élaboration des cibles et objectifs définis, se traduisant par un projet de programme d'activités clés (avec des échéances et des responsables pour leur mise en œuvre) conçu pour atteindre les objectifs et mener au changement désiré ;

6. Chiffrage des activités et réformes envisagées - Calcul des coûts financiers et non financiers nécessaires à la réalisation des actions planifiées et parvenir au changement attendu, et identification des sources de financement pour les actions décidées ;

7. Suivi et réalisation de rapports - Développement et exécution d'un processus et d'un système permettant l'évaluation régulière des progrès par rapport aux provisions, pour permettre une mise en œuvre efficace et efficiente ;

8. Évaluation - Élaboration et mise en œuvre d'un système fondé sur des indicateurs pour apprécier si ont été atteints les objectifs de réforme initialement envisagés par rapport aux problèmes définis dans l'état originel de la situation, afin d'ajuster, affiner ou arrêter certaines réformes, ou de soutenir le développement de nouvelles phases de réformes.

En plus des phases du cycle énumérées ci-dessus, il existe un aspect complémentaire important des stratégies de RAP : à savoir le cadre de gestion et de coordination de la RAP. Les stratégies de RAP doivent être conçues de telle façon que chaque élément du cycle ci-dessus puisse fonctionner de manière efficace et efficiente, et que chaque phase ou étape du cycle soit menée en concertation avec les diverses parties prenantes du gouvernement ou extérieures à celui-ci. Cette concertation permet d'aider à identifier les problèmes et les solutions et en débattre, ainsi qu'à accroître l'acceptation, la crédibilité et le réalisme du processus de réforme d'un pays. Idéalement, cette concertation devrait permettre d'aborder la question de la parité hommes-femmes pendant la consultation et en lien avec le développement de la stratégie (particulièrement dans le cas des stratégies liées à la RAP). L'analyse des impacts du point de vue de la parité (à la fois durant le processus de développement de la stratégie et au cours de sa mise en œuvre) devrait être prise en compte lors de la planification des réformes dans tous les secteurs?.

Au cours de l'élaboration de stratégies sectorielles complexes, impliquant un certain nombre d'institutions et de parties prenantes, il est également important de veiller à ce qu'il y ait une structure de gestion et de coordination adéquate reflétant les complexités institutionnelles et thématiques.

Il est également important de souligner que la RAP ainsi que les réformes sectorielles doivent être menées avec le maximum de transparence en assurant la communication sur les réformes et leur visibilité, à la fois à l'intérieur et à l'extérieur de l'administration, en programmant une communication bien ciblée et pertinente sur les

\footnotetext{
${ }^{9}$ Pour une lecture complémentaire sur la gouvernance sexospécifique, voir: OCDE (2018), Toolkit for Mainstreaming and Implementing Gender Equality, Paris, http://www.oecd.org/gender/governance/toolkit.
} 
objectifs de la réforme et leur réalisation ${ }^{10}$. La participation des citoyens est cruciale tout au long de l'élaboration et de la mise en œuvre des stratégies, et ne peut être assurée qu'avec l'engagement explicite du gouvernement à mener ses affaires d'une manière ouverte, compréhensible et transparente, en fournissant des informations, sur internet ou ailleurs, qui décrivent minutieusement les initiatives gouvernementales dans une langue compréhensible pour les citoyens ${ }^{11}$.

Le cycle du processus de stratégie est illustré par le Schéma 1.

Il est important de noter que cette approche par cycle n'est qu'une manière de présenter le processus complexe de développement et de mise en œuvre des stratégies. Diverses autres approches existent, mais l'un des aspects qui leur est commun avec l'approche par cycle est qu'elles préconisent toutes un processus global, inclusif et complexe pour la conception de politiques et de stratégies. Des études récentes présentent de bons arguments en faveur de l'application, par les gouvernements, d'une approche conceptuelle ou systémique lors de la préparation et de la mise en œuvre de politiques ou stratégies nouvelles ${ }^{12}$.

10 Pour plus d'informations sur l'importance de la communication sur la réforme, et sur l'approche de cette communication, voir également: Commission européenne (2017), Lignes directrices le l'appui budgétaire, Bruxelles, https://ec.europa.eu/europeaid/sites/devco/files/bsg_fr_web_version_20180608-v2.pdf pp. 6264.

${ }^{11}$ Le besoin d'une élaboration des politiques et d'une gouvernance inclusives et transparentes est au cœur du Partenariat pour une gouvernance inclusive (PGO), une initiative rejointe par presque 100 pays et villes depuis son lancement en 2011, qui s'engagent activement à rendre leur administration plus ouverte, responsable et attentive aux citoyens. Le PGO a développé une série d'outils utiles pour les décideurs et les parties prenantes, afin de mettre ces engagements en œuvre. Pour plus d'informations, voir https://www.opengovpartnership.org. Pour plus d'informations sur l'engagement de l'OCDE envers le gouvernement ouvert, voir les Recommandations du Conseil sur le gouvernement ouvert, adoptées le 14 décembre 2017 par le Conseil de l'OCDE, et OCDE (2016), Gouvernement ouvert : Contexte mondial et perspectives, Éditions OCDE, Paris, https://doi.org/10.1787/9789264280984-fr.

12 Pour des lectures complémentaires sur la pensée systémique et son application dans les réformes de la gouvernance, voir OCDE (2017), Systems Approaches to Public Sector Challenges: Working with Change, Éditions OCDE, Paris, https://doi.org/10.1787/9789264279865-en et OCDE (2017), Fostering Innovation in the Public Sector, Éditions OCDE, Paris, https://doi.org/10.1787/9789264270879-en . 
Schéma 1. Phases du processus de stratégie

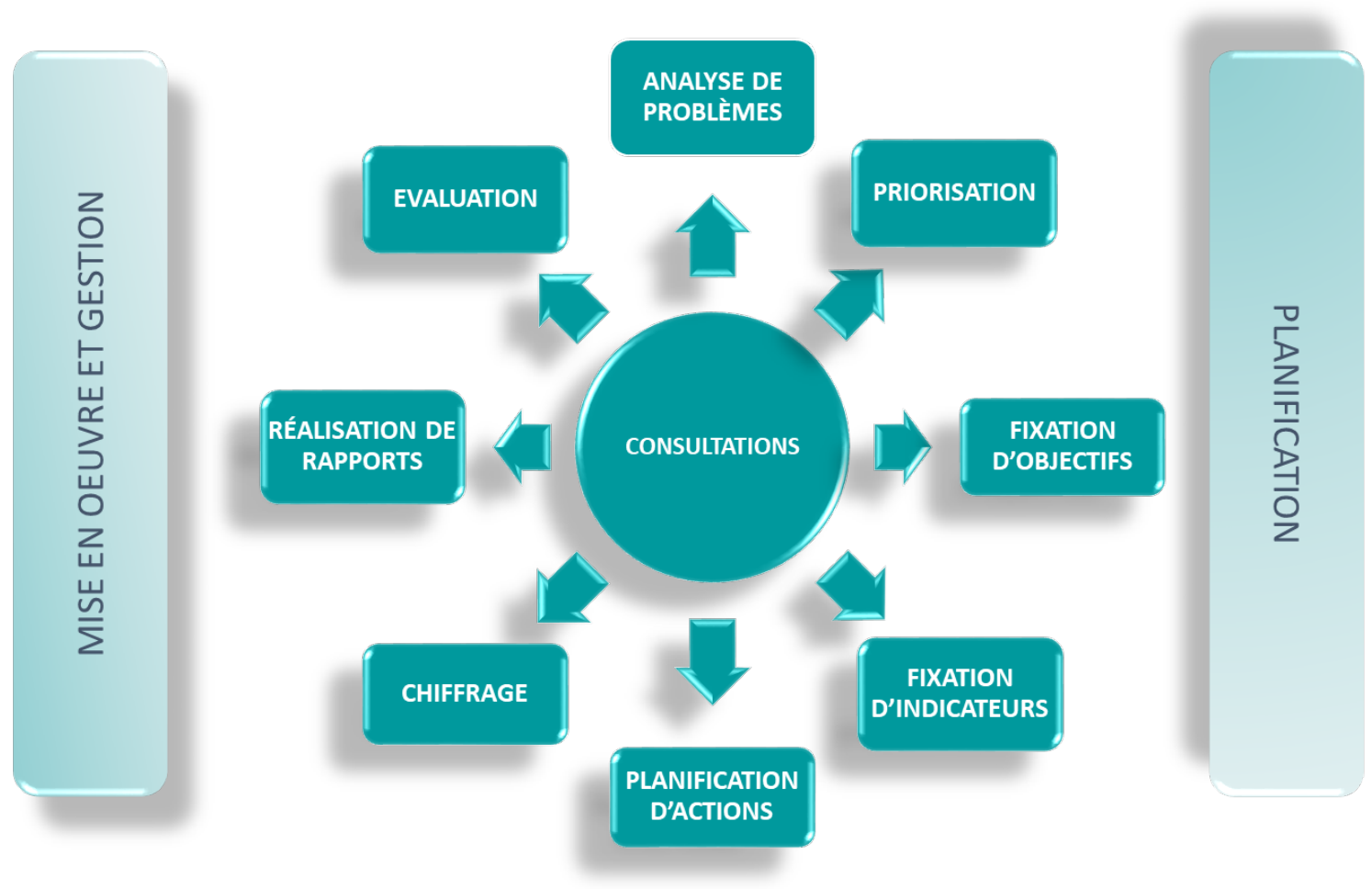

Pourquoi la planification préalable est-elle importante pour l'élaboration d'une stratégie?

Il existe un lien étroit entre la réussite des réformes et le soin avec lequel celles-ci ont été planifiées. En outre, les personnes chargées de l'élaboration d'une stratégie ont besoin d'un délai suffisant pour examiner les problèmes en profondeur (en se basant sur des données), consulter et impliquer ceux qui seront affectés par la stratégie, déterminer les bons objectifs et définir les indicateurs les plus appropriés, prévoir un ensemble optimal d'actions clés et évaluer leur coût avec précision. Par souci d'assurer un processus d'élaboration inclusif et de garantir la crédibilité et l'appropriation requises pour la réforme, les parties prenantes externes (autres institutions concernées et acteurs non gouvernementaux) devront disposer de suffisamment de temps pour être impliquées de manière efficace dans le processus d'élaboration de la stratégie.

Hâter le processus d'élaboration de la stratégie peut facilement avoir pour résultat une analyse incomplète, un manque de clarté sur les directions requises pour la réforme (objectifs), un ensemble mauvais ou incomplet d'actions clés, et des réformes peu crédibles, avec pour conséquence un mécontentement des parties prenantes internes et externes. Au pire, un mauvais processus d'élaboration peut déboucher sur de faibles taux de mise en œuvre des activités envisagées, ou faire plus de mal que de bien au fonctionnement de l'administration publique, du secteur concerné ou des personnes affectées par la stratégie. C'est pourquoi il est important, afin d'élaborer une stratégie de bonne qualité et crédible, il est important d'accorder suffisamment de temps et de ressources institutionnelles à sa préparation et à son adoption.

Si l'élaboration d'une nouvelle stratégie ne démarre que lorsque la stratégie précédente s'est achevée (ou juste avant), la conséquence la plus probable sera un temps mort dans 
le processus de réforme. Cela peut engendrer divers risques tels qu'une instabilité institutionnelle, l'arrêt de la dynamique de réforme, des discontinuités de financement, ou même un manque de crédibilité du processus global de réforme. De même, si l'administration n'engage pas le processus d'élaboration de la stratégie suffisamment tôt, les gouvernements pourraient être confrontés à des pressions politiques ou à d'autres demandes extérieures pour agir rapidement, et par conséquent exiger une élaboration de stratégie plus rapide qu'il ne faudrait, menant ainsi de la même façon à des problèmes de qualité et de crédibilité du produit final.

En moyenne, selon l'expérience de SIGMA, l'élaboration et l'adoption d'une RAP ou d'une stratégie sectorielle prend entre 9 et 12 mois. Dans certains cas, cela peut même être plus long, selon le contexte. Ainsi, l'élaboration d'une stratégie (ou la révision d'une stratégie existante) devrait débuter au moins 12 mois avant le début prévu de sa mise en ouvre, ou l'expiration d'une stratégie existante.

\section{Questions clés pour la planification du processus d'élaboration de stratégie}

Lors de la planification du processus d'élaboration d'une RAP ou d'une stratégie sectorielle, les responsables devraient prendre en compte les éléments suivants :

- Les méthodes de recueil et d'analyse des données qui seront utilisées (en particulier l'établissement de valeurs de référence pour les indicateurs), car des méthodes différentes exigent des délais d'exécution différents ;

- Les ressources humaines et financières requises pour l'élaboration de la stratégie, selon que celle-ci sera développée uniquement à partir de ressources internes ou que certaines des tâches devront être externalisées ${ }^{13}$;

- Les processus, méthodes, périmètres et compositions des structures institutionnelles d'élaboration de stratégie ;

- Les processus, méthodes et publics cibles de la concertation avec les parties prenantes externes (non gouvernementales et inter institutionnelles), dont les citoyens, en accord avec les critères du pays concernant la participation des parties prenantes.

Tous les facteurs cités ci-dessus ont un impact sur le calendrier du processus d'élaboration de la stratégie. Ils peuvent raccourcir ou prolonger l'élaboration d'une stratégie, et doivent donc être soigneusement étudiés à l'avance. Pour faciliter le processus, il peut être utile d'établir un document résumant les décisions prises (par exemple un relevé de conclusions) mentionnant les étapes principales, les parties prenantes les plus importantes, la composition des organes de travail, le calendrier

\footnotetext{
${ }^{13}$ Pour plus d'informations sur les défis liés aux compétences nécessaires à une fonction publique hautement performante, et sur les tendances récentes pour relever ces défis, voir : OCDE (2017), Skills for a High Performing Civil Service, Examens de l'OCDE sur la gouvernance publique, Éditions OCDE, Paris, https://doi.org/10.1787/9789264280724-en. Le Groupe de travail de l'OCDE sur l'emploi et la gestion publics (Public Employment and Management Working Party - PEM) identifie les tendances actuelles et les bonnes pratiques concernant les capacités, les compétences et la performance. Le PEM est un forum international collaboratif d'experts chevronnés cherchant à relever les défis actuels concernant les services publics et la réforme de la fonction publique. Il entreprend des analyses comparatives sur des questions liées à la gestion stratégique et à la rémunération de la fonction publique, qui fournissent aux gouvernements des données uniques pour étayer leurs programmes de réforme.
} 
général de l'intégralité du processus, les besoins en ressources pour toutes les phases d'élaboration de la stratégie, et le détail des consultations et communications prévues ${ }^{14}$.

En ce qui concerne la RAP, il est également important de décider en avance si le pays veut avoir un seul document stratégique de RAP ou plusieurs stratégies liées à la RAP. Il n'y a pas qu'une seule bonne manière d'établir le cadre des documents stratégiques de la RAP. Le nombre de stratégies liées à la RAP dépend de la structure administrative/institutionnelle, ainsi que de la complexité et de l'ambition de la RAP. Par exemple, la réforme de la gestion des finances publiques (GFP) est un domaine complexe, nécessitant souvent une stratégie séparée. Dans le cas de plusieurs stratégies, il est particulièrement important de s'assurer que l'information et la gestion des divers domaines de la RAP couverts par différentes stratégies passent par un canal d'information (système et processus de suivi et réalisation de rapports) et une structure de gestion uniques ou travaillant en bonne intelligence. C'est ainsi que les institutions qui s'occupent de différents aspects de la réforme pourront coordonner efficacement leurs actions, leurs solutions et l'allocation des ressources, à la fois pour l'élaboration et pour la mise en œuvre des divers documents stratégiques.

14 Pour plus d'informations sur la https://ec.europa.eu/europeaid/node/1579 series/minisite/list-available-publications gestion par programme ou par projet, voir : et https://europa.eu/capacity4dev/t-and-m- 


\section{Analyse de problème}

La première phase du processus de l'élaboration de la stratégie est l'analyse de la situation actuelle, dans le but d'identifier les problèmes et leurs causes, ainsi que les défis et opportunités de la réforme. Pendant cette phase, l'équipe chargée de concevoir la stratégie doit recueillir des éléments probants concernant les problèmes, défis et opportunités identifiés et les fournir aux décideurs lors des différentes étapes du processus. Il s'agit des faits, données et informations disponibles qui corroborent ou non les opinions, jugements ou propositions émis.

C'est une phase cruciale pour l'élaboration de la stratégie, car les résultats de l'analyse sont utilisés plus tard pour la hiérarchisation, la fixation d'objectifs et l'identification des actions. La qualité de l'analyse est déterminante pour la qualité de la stratégie : la profondeur de l'analyse déterminera quels problèmes peuvent être identifiés et attestés, et aura des conséquences décisives sur les objectifs de la stratégie. Il est donc nécessaire de soigneusement prévoir quelles méthodes d'analyse et de recueil des données seront choisies afin d'évider les biais et l'imprévision et pour garantir l'implication des parties prenantes clés.

Les résultats de l'analyse de problèmes seront ensuite résumés dans la stratégie arrêté, et selon la méthodologie requise pour l'élaboration de la stratégie, soit dans une section distincte (comme c'est le cas pour les Stratégies de RAP de l'Albanie ${ }^{15}$, de $1^{\prime}$ Ukraine $^{16}$ et du Monténégro ${ }^{17}$, et pour le Programme national des réformes majeures de la Tunisie 2016-2020 ${ }^{18}$ ), soit comme partie de la section détaillant les objectifs de la réforme (cas des Stratégies de RAP de la Moldavie ${ }^{19}$ et de la Géorgie ${ }^{20}$ ). L'analyse devra être concise et précise, et ne présenter que de l'information analytique, et non descriptive. Les informations supplémentaires et les données justificatives peuvent, si besoin, être présentées dans une annexe séparée du document de stratégie.

Néanmoins, la portée de l'analyse devrait être large et exhaustive pour permettre de saisir tous les aspects pertinents des questions de politique publique en jeu. Plus tard, pendant la phase de hiérarchisation et de cadrage de la stratégie, les éléments pertinents

15 Cross-cutting PAR Strategy 2015-2020, avril 2015. http://dap.gov.al/images/DokumentaStrategjik/PAR_Strategy_2015-2020_English.pdf.

16 Strategy of Public Administration Reform in Ukraine for 2016-2020, juin 2016, http://zakon2.rada.gov.ua/laws/show/ru/474-2016-\%D1\%80\#n9.

17 Public Administration Reform Strategy in Montenegro 2016-2020, juillet 2016, http://www.mju.gov.me/ResourceManager/FileDownload.aspx?rid=268749\&rType=2\&file=P UBLIC\%20ADMINISTRATION\%20REFORM\%20STRATEGY\%20IN\%20MONTENEGRO \%202016-2020.pdf.

18 Programme des Réformes Majeures 2016-2020, janvier 2016, https://www.tustex.com/sites/default/files//PNRM2016_2020.pdf.

19 Public Administration Reform Strategy 2016-2020, juin 2016, https:/cancelaria.gov.md/sites/default/files/document/attachments/strategie_actualizata_par_str ategy_2016-2020_30jun16.pdf.

20 Public Administration Reform Roadmap 2020, mai 2015,

http://gov.ge/files/425_49309_322150_15.07.21-

PublicAdministrationReformRoadmap2020(Final)(1).pdf. 
clés d'une analyse plus large servent à expliquer pourquoi, le cas échéant, certains problèmes analysés ne sont pas traités dans la stratégie. Cependant, afin d'élaborer une stratégie pertinente et crédible, il est indispensable d'avoir un aperçu analytique exhaustif de la situation constatée, identifiant les principaux défis et les causes des problèmes. Ce résultat sera d'autant plus facilement atteint que l'équipe travaillant sur l'analyse est constituée de façon à permettre la représentation d'avis variés, et est multidisciplinaire, à la fois en termes de l'étendue des connaissances de ses membres et de leur provenance sectorielle ou institutionnelle.

Les principaux résultats de l'analyse devraient être la liste et la description, fondées sur des éléments probants, des :

- principaux résultats atteints par la stratégie précédente et celle en cours (avec une attention spéciale portée à l'achèvement des réformes en question);

- principaux problèmes ou obstacles et leurs causes profondes

Les résultats de l'analyse peuvent être résumés en phrases courtes et bien ciblées, relevant les principaux problèmes, soulignant certaines données probantes et expliquant la nature du problème. De tels résumés ne nécessitent pas forcément de longues explications, comme les quelques exemples de l'encadré 1 le montrent.

\section{Encadré 1. Exemples de descriptions de problème liés à la RAP}

Faible lien entre planification des politiques et processus budgétaire

L'élaboration des politiques est, dans sa majeure partie, séparée du processus budgétaire. Le Programme du gouvernement ainsi que $80 \%$ des documents de planification à court terme et $30 \%$ des documents de planification à moyen et long terme n'ont pas de lien direct avec le document de Cadre économique à moyen terme ou budget annuel; ils ne contiennent pas non plus d'estimations financières relatives à la mise en œuvre des mesures envisagées. Cette situation est due au fait qu'il n'existe pas d'exigence formelle claire de fournir de telles informations ; de plus, la capacité des ministères à fournir de telles estimations fait sévèrement défaut.

\section{Organisation complexe de l'administration publique}

Les principales défaillances sont liées à la diversité des statuts et fonctionnements des organisations, ainsi qu'à un contrôle insuffisant de la légalité et de l'efficacité de leur travail. Il n'existe pas de typologie claire des organisations du secteur public, ni de critère précis pour définir leur statut. L'absence d'un tel système conduit à avoir plus d'une douzaine de types d'organisations sans critères bien définis quant à leur fonctionnement ou à leurs obligations de rendre compte.

Manque d'exigences claires dans le domaine de l'accès à l'information

La Loi sur l'accès à l'information est incomplète, elle ne décrit pas clairement la nature des informations que les ministères et agences doivent publier, et l'autorité responsable de sa mise en œuvre n'a pas encore été désignée. Par conséquent, au moins $50 \%$ des institutions publiques n'ont pas respecté les dispositions de base de la Loi. Ces institutions ne publient pas de données sur l'exécution du budget, ni de rapports d'activité, ni des 
informations sur leurs initiatives en matière de politique publique. Le cadre légal actuel justifie le manque d'initiatives proactives, notamment le manque de volonté des institutions pour publier des informations relatives à leurs activités.

\section{Tarifs des services publics arbitraires et déraisonnables}

Il n'existe pas de principe uniforme et transparent pour normaliser l'établissement des tarifs des services fournis. Les prix des services sont considérés par la plupart des citoyens comme arbitraires et déraisonnables. L'absence de principes et de directives pour fixer le prix des services payants se traduit par des tarifs plus élevés. Par conséquent, les services payants sont généralement considérés comme une source de revenus supplémentaires pour les institutions publiques, enregistrés comme « moyens spéciaux ». En outre, la faiblesse du cadre légal et l'absence de clarté sur les éléments constitutifs des services compliquent le calcul de leur prix de revient et rendent l'évaluation et la fixation objectives des tarifs difficile.

L'analyse des problèmes aide à les comprendre, à déceler leurs origines, à évaluer leur gravité et leurs causes profondes, et à décider s'ils requièrent une action immédiate. Il est important de s'appuyer sur des données pour déterminer la nature des problèmes et leur gravité. Par exemple, les données peuvent montrer des écarts de performance par rapport à d'autres institutions, à d'autres régions ou à des objectifs précis ; elles peuvent aussi révéler des écarts plus ou moins importants par rapport aux structures comparables.

Il existe plusieurs options différentes pour choisir qui mènera l'analyse, selon le temps et le budget disponibles, le niveau d'expertise requis, ainsi que la culture et la tradition de l'administration concernée. L'encadré 2 évalue les modalités généralement retenues pour conduire l'analyse. 
Encadré 2. Avantages et limites des modalités habituellement retenues pour analyser les problèmes (exemple de la RAP)

OPTION 1 : Analyse en interne

Des fonctionnaires des ministères et agences conduisent l'analyse.

Présider par l'institution chef de file de la RAP, un groupe de travail composé de fonctionnaires appartenant aux institutions clés avec et ayant des compétences et une expertise complémentaires, avec l'apport éventuel d'experts externes, peut être créé pour conduire l'analyse. La clé de la réussite d'une analyse interne réside dans une bonne conception de l'analyse, avec une sélection appropriée des questions et des sources d'information afin d'obtenir un tableau impartial et révélateur.

Avantages : meilleure vision interne des problèmes ou lacunes, renforcement professionnel de l'expertise des fonctionnaires impliqués dans le processus.

Limites : plus long (les fonctionnaires ont habituellement d'autres tâches à remplir parallèlement à l'analyse), peu d'opportunités d'appliquer des méthodes d'analyse sophistiquées, manque d'autocensure interne, plus grand risque de biais.

Exemples : l'analyse interne a été utilisée par le gouvernement monténégrin lors de la préparation de sa stratégie de RAP 2016-2020. Le gouvernement du Kosovo a mené une analyse interne lors de l'élaboration de sa Stratégie de modernisation de l'administration publique 2015-2020 21 et de la Stratégie pour l'amélioration de l'élaboration et de la coordination des politiques au Kosovo 2017-202122.

* Cette désignation est sans préjudice des positions sur le statut et est conforme à la résolution 1244/99 du Conseil de sécurité des Nations unies ainsi qu'à l'avis de la Cour internationale de Justice sur la déclaration d'indépendance du Kosovo.

21 Strategy on Modernisation of Public Administration, 2015-2020, septembre 2015, http://www.kryeministri-ks.net/repository/docs/Strategy-for-Modernisation-of-PA-20152020.pdf.

22 Strategy for Improving Policy Planning and Coordination in Kosovo (Integrated Planning System) 2017-2021, décembre 2016, http://www.kryeministriks.net/repository/docs/Strategy_for_Improving_Policy_Planning_and_Coordination_in_Kosov o_2017-2021.pdf. 


\section{OPTION 2 : Externalisée}

Une ONG ou un prestataire de service privé est chargé de mener l'analyse.

L'institution chef de file de la RAP doit rédiger le cahier des charges $(\mathrm{CdC})$ pour cibler correctement l'étendue de l'étude et sa méthodologie, ce qui exige une expertise.

Avantages : permet d'utiliser des approches méthodologiques plus sophistiquées ou complexes pour arriver à des données plus concrètes lorsque l'expertise interne n'est pas disponible, peut être plus rapide que l'analyse interne.

Limites : moins d'appropriation interne; information limitée sur les problèmes graves non perceptibles par un analyste externe ; requiert des ressources financières supplémentaires; limite le développement des compétences internes nécessaires.

Exemples: Stratégie transversale de RAP de l'Albanie 2015-2020, Stratégie de réforme de l'administration publique en République de Serbie $^{23}$ et Stratégie de RAP de la Moldavie 2016-2020.

\section{OPTION 3 : Combinaison}

Des fonctionnaires des ministères et agences mènent l'analyse initiale. Une ONG ou un prestataire de service du secteur privé est engagé pour mener une analyse plus en profondeur (ou complémentaire) dans certains domaines, pour recueillir des données supplémentaires ou pour apporter un avis extérieur afin de vérifier la qualité et les conclusions de l'analyse en interne.

L'institution chef de file de la RAP doit développer un CdC pour cibler correctement la portée et définir la méthodologie; ceci requiert une expertise.

Solution alternative, il est possible d'associer dans un groupe de travail mixte des fonctionnaires, experts, et ONGs, les experts préparant les documents et analyses de référence, puis discutant et finalisant l'analyse de problèmes lors des réunions du groupe de travail avec les fonctionnaires.

Bénéfices : permet d'améliorer l'approche et le contrôle de la qualité de l'analyse initiale, pour s'assurer que les questions principales ont été traitées ; occasion d'utiliser des approches méthodologies plus solides pour aboutir à des données plus concluantes.

Limites : plus long; requiert des ressources financières additionnelles et des efforts de coordination supplémentaires ; limite le développement des compétences internes nécessaires ; l'appropriation risque d'être limitée par rapport à un processus qui aurait été mené uniquement en interne.

23 Public Administration Reform Strategy in the Republic of Serbia, janvier 2015, http://www.mduls.gov.rs/english/reforma-javne-uprave.php. 
Exemples : Ce processus combiné a été utilisé en Bosnie-Herzégovine pour l'analyse préalable à l'élaboration d'une stratégie de RAP à l'échelle du pays en 2016 et $2017^{24}$.

Une bonne analyse prend du temps, en général entre deux et six mois, parfois même plus. Le temps alloué à la réalisation de l'analyse prévue des problèmes dépend des conditions particulières du processus de prise de décision, des ressources disponibles et de la complexité des processus de consultation externe et interne nécessaires pour élaborer et valider les conclusions de l'analyse. Surtout, celles-ci dépendent de l'existence de données suffisantes et fiables.

Quelle que soient les modalités choisies pour l'analyse, il est important d'impliquer les diverses parties prenantes et de les consulter, à la fois pendant le déroulement de l'analyse et sur ses résultats : décideurs, responsables, personnel dédié à la mise en œuvre, utilisateurs externes du service, ONGs et citoyens. Une large consultation permettra de recueillir les problèmes sur le terrain et d'élaborer des mesures y répondant, ce qui est pertinent pour les groupes en définitive ciblés par les réformes, c'est-à-dire les utilisateurs des services ou les bénéficiaires directs de la stratégie.

La communication sur les résultats de l'analyse est également importante. Elle devrait également contribuer à donner l'orientation de la réforme et à éviter les tentations de rejet.

\subsection{Planification de l'analyse}

Étant donné que l'analyse peut nécessiter non seulement le recueil des données existantes mais aussi de données complémentaires, et peut reposer sur une combinaison de plusieurs méthodes de recueil de données, il est nécessaire de planifier l'analyse suffisamment en avance pour garantir son achèvement dans le délai prévu. La planification de l'analyse implique de :

- définir le champ de l'analyse ;

- poser les questions pertinentes par rapport au champ retenu (préparer une série de questions) ;

- sélectionner les principales méthodes de recueil des données et leurs sources ;

- définir quelles sont les principales institutions et parties prenantes impliquées ainsi que leurs responsabilités respectives.

Une bonne analyse de l'état actuel de la situation (se concentrant sur l'identification des problèmes qui appellent une réforme ou une intervention) est importante pour définir correctement la portée de la stratégie de la réforme à venir, ses priorités et les objectifs du processus de changement. Les domaines (ou le champ) de l'analyse peuvent être fondés sur :

- les piliers d'une RAP ou d'une stratégie sectorielle en cours ou achevée ;

- les Principes d'administration publique (pour une stratégie de RAP);

${ }^{24}$ Au moment de la publication de ce Manuel, la Stratégie de RAP de la Bosnie-Herzégovine n'a pas été adoptée par tous les niveaux administratifs principaux en Bosnie-Herzégovine, mais SIGMA a été impliqué dans l'élaboration de la version préliminaire de la stratégie, et est donc informé de l'utilisation d'une méthode combine pour l'élaboration de l'analyse des problèmes. 
- les problèmes jugés les plus pressants par les parties prenantes clés ;

- les changements dans le programme politique du (nouveau) gouvernement.

Cependant, pour chacune de ces options, le champ de l'analyse devrait être aussi exhaustif et objectif que possible pour permettre de dresser un tableau complet des résultats obtenus, des principaux défis et des problèmes décelés dans le champ retenu, et pour aider à les hiérarchiser par la suite.

Avant d'entreprendre l'analyse, il est nécessaire de faire un travail préparatoire, à la fois pour éviter les chevauchements d'activité et pour préparer les tâches analytiques proprement dites. Au cours de cette préparation, l'équipe chargée de l'analyse doit identifier les rapports de suivi et d'évaluation sur la mise en œuvre des stratégies précédentes, les analyses externes (par exemple les évaluations SIGMA sur la RAP et les rapports de la CE sur l'élargissement, les rapports d'évaluation par les pairs dans différents secteurs, les analyses de $1^{\prime} \mathrm{OCDE}^{25}$, etc.), les données disponibles relevant du champ de l'analyse, et la liste des parties prenantes clés.

L'encadré 3 présente une série de propositions de questions pour l'analyse de problèmes. Il ne s'agit pas d'une liste exhaustive, mais elle peut être utilisée comme base pour la préparation d'une liste plus complète de questions clés.

\section{Encadré 3. Liste de questions indicatives pour l'analyse de problèmes}

1. S'agissant des principaux défis et problèmes liés à la mise en œuvre des stratégies précédentes :

a. Quels sont les principaux défis et problèmes qui, selon les destinataires des stratégies précédentes, n'ont pas été traités, ou l'ont été insuffisamment? D'autres défis du même type, ou similaires, sont-ils apparus depuis le début de la mise en œuvre des stratégies précédentes?

b. De quels aspects de la réforme les institutions chargées de la mettre en œuvre se plaignent-elles le plus ?

c. Quels sont les avis des parties prenantes sur la mise en œuvre des stratégies précédentes ou sur les principaux problèmes liés à la RAP, à divers domaines de la RAP ou à un secteur particulier ?

2. En se basant sur des données, y compris tout rapport d'évaluation ou de suivi interne ou externe, quels sont les plus grandes écarts de performance constatés sur les réformes précédentes, ou sur le fonctionnement de l'administration publique, dans son ensemble ou dans un secteur particulier? Une évaluation plus large des potentiels peut aussi porter, entre autres, sur les insuffisances des processus de planification et sur les chevauchements de compétences des différents organismes.

${ }^{25}$ Dans le cas des réformes de la gouvernance publique, il est possible d'obtenir une perspective comparative sur les principaux problèmes de gouvernance de OCDE (2017), Panorama des administrations publiques 2017, OECD Publishing, Paris. https://www.oecdilibrary.org/governance/panorama-des-administrations-publiques-2017_gov_glance-2017-fr 
3. S'agissant des principales causes de problèmes et des insuffisances relevés par les données disponibles (provenant d'évaluations internes et externes) :
a. Pourquoi les objectifs et cibles de performance n'ont-ils pas été atteints?
b. Pourquoi les actions n'ont-elles pas été mises en œuvre ??
c. Quelles activités non planifiées ont-elles été entreprises, et pourquoi?

4. Quels sont, d'après les organismes chargés de la mise en œuvre et les principales parties prenantes, bénéficiaires compris, les causes des problèmes et insuffisances?

5. D'après les données disponibles et les réclamations des organismes chargés de la mise en œuvre ou des parties prenantes, bénéficiaires compris, le problème ou l'écart constaté sont-ils significatifs ?

6. Quelle est la performance du pays dans les divers domaines de l'administration publique ou dans un secteur particulier, comparée à d'autres (pays similaires, leaders régionaux ou internationaux)?

7. Quelles sont les raisons systémiques de l'écart de performance par rapport à d'autres pays ?

8. Quelles actions ou réformes peuvent-elle s'inspirer d'autres pays ou d'organismes remarquablement performants, et quelles sont les limites d'une telle transposition?

\subsection{Outils analytiques communément utilisés}

Le processus analytique peut soit employer des nombreux outils et méthodes, ou bien être simplifié et utiliser moins d'outils.

Un processus simplifié peut être privilégié lorsqu'il y a insuffisance de compétences internes, d'expertise analytique et de ressources financières. Dans ces cas, il est recommandé d'identifier les principaux domaines d'analyse et de commencer simplement à énumérer et décrire les résultats atteints, les lacunes, les problèmes et leurs causes profondes. Il faut souligner que, lorsque le processus analytique est simplifié de cette façon, il est particulièrement important d'avoir dès le départ impliqué l'ensemble des parties prenantes internes et externes et mené des consultations larges et bien ciblées et afin de permettre que soient partagées et validés les résultats de l'analyse : l'identification des problèmes majeurs et de leurs causes profondes. La consultation et la participation du public peuvent également être utilisées pour enrichir encore l'analyse par l'apport de données, informations et avis supplémentaires.

L'utilisation de méthodes et outils spécifiques exige des ressources, une préparation intensive et de la coordination. Les outils les plus communément utilisés pour structurer et organiser l'analyse sont : 
- L'analyse des facteurs politiques, économiques, sociaux, technologiques et légaux (PESTEL) ${ }^{26}$

- Analyse des forces, faiblesses, opportunités et menaces (Strengths, Weaknesses, Opportunities and Threats - SWOT)

- Analyse par arbre à problèmes

L'analyse PEST $(\mathbf{E}) \mathbf{L}^{27}$ est un cadre pour l'analyse de l'environnement externe de la politique concernée. Elle comprend une liste de contrôle des domaines à étudier lors de l'analyse de ces facteurs. Elle est utilisée pour déterminer quels facteurs externes faciliteront la mise en œuvre de la politique retenue, ou au contraire y feront obstacle. Ces éléments seront ensuite traduits en opportunités ou menaces dans l'analyse SWOT, ou utilisés indépendamment. Certaines des questions qui peuvent être utilisées pour structurer une analyse PESTL sont présentées dans l'encadré 4, et l'encadré 5 présente un exemple du résumé d'une analyse PESTL théorique.

${ }^{26}$ Pour des informations complémentaires sur ces méthodes et leur utilisation (en anglais), voir :

- PEST(E)L sur https://www.mindtools.com/pages/videos/ pest-transcript.htm et https://www.mindtools.com/pages/article/newTMC 09.htm;

- SWOT sur http://diytoolkit.org/tools/swot-analysis-2/ et https://www.mindtools.com/pages/article/newTMC_05.htm;

- Arbre/diagramme à problèmes sur http://diytoolkit.org/tools/causes-diagram/ ; et

- Définition de problèmes sur http://diytoolkit.org/tools/problem-definition-2/.

${ }^{27}$ Dans sa forme classique, cette méthode couvre également les facteurs écologiques (second $\mathrm{E}$ de PESTEL pour « environnemental »), mais cela n'est généralement pas utilisé lors de l'analyse $\mathrm{du}$ fonctionnement et de la performance des administrations publiques. 
Encadré 4. Questions pour structurer une analyse PESTL

\section{Politique}

- Quel est l'impact du calendrier politique (par exemple élections, programmes gouvernementaux et priorités politiques) sur la stratégie?

- Existe-t-il des engagements politiques (nouveaux) qui devraient être traités par la politique retenue?

- En quoi d'autres politiques publiques sectorielles (par exemple fiscalité, concurrence et développement régional) peuvent-elles affecter la stratégie?

\section{Économique}

- En quoi les évolutions économiques actuelles ou à venir (consommation, emploi, inflation, niveau de revenus, etc.) peuvent-elles affecter la politique retenue et son groupe cible?

- La position actuelle du groupe cible va-t-elle se détériorer, s'améliorer ou se stabiliser, et cela requerra-t-il de nouvelles solutions?

\section{Social}

- Comment la démographie, les obstacles culturels et les niveaux d'éducation et la santé peuvent-ils affecter la politique retenue et son groupe cible?

- Quels changements et quelles solutions faudrait-il apporter?

\section{Technologique}

- En quoi la technologie peut-elle affecter positivement ou négativement la politique retenue et son groupe cible, de manière positive ou négative ?

- Existe-t-il de nouvelles technologies qui pourraient être utilisées pour rendre plus efficace et plus efficiente la politique retenue ?

Légal

- Existe-t-il des blocages juridiques retardant la mise en œuvre des activités et réformes envisagées ? Où se trouvent-ils ?

- Quelle est l'importance des obligations réglementaires dans un domaine donné, et existe-t-il des blocages juridiques affectant la mise en œuvre des réformes?

\section{Environnemental}

- Comment les facteurs environnementaux (par ex. changement climatique ou pollution) affectent-ils la politique retenue et son groupe cible?

- Quels changements et quelles solutions faudrait-il apporter? 


\section{Encadré 5. Exemple de résumé d'une analyse PESTL théorique} (basé sur une RAP)

\section{Politique}

- Les ambitions de mise en place de la gouvernance électronique, énoncées dans le nouveau Programme gouvernemental, offrent de nombreuses opportunités nouvelles pour l'adoption d'un programme de réformes innovant dans les domaines de la fonction publique, de la gestion des finances publiques et de la prestation de services ;

- La priorité donnée au renforcement de l'équilibre budgétaire et à la réduction de l'évasion fiscale permettra de faire des réformes significatives dans le domaine de l'administration fiscale, où les outils électroniques peuvent être utilisés ;

- Il n'existe pas d'engagement politique clair pour réformer la coordination des politiques et l'obligation de rendre compte. Ceci démontre la nécessité de rechercher un accord et de construire une large coalition de soutiens politiques ;

- L'incitation forte donnée par la CE pour progresser dans la mise en place d'une RAP est un encouragement à renouveler le cadre stratégique de la RAP.

\section{Économique}

- Une hausse prévue de la croissance économique (estimation de $+4 \%$, et de $+5,5 \%$ dans les deux ans) permettra, à moyen terme, des réformes de l'administration publique plus ambitieuses ;

- Les lacunes de financement des réformes prévues par la RAP peuvent être significativement réduites en réaffectant les ressources budgétaires provenant des économies dues à une efficience accrue et en hiérarchisant les réformes.

Social

- Étant donné le vieillissement de la population, il y aura besoin à long terme d'augmenter les dépenses telles que les retraites et les soins de santé, ce qui occasionnera de plus fortes contraintes budgétaires dans d'autres domaines de réforme, y compris la RAP ;

- Les réformes de l'administration publique peuvent significativement contribuer à mettre en œuvre des réformes des politiques sociales en améliorant la prestation de services, par exemple en organisant des projets pilotes de prestation de services dans le secteur social ;

- L'évolution vers le libre-service à travers les outils électroniques nécessite que soit améliorée la pratique de l'informatique, pour permettre aux citoyens d'utiliser des services électroniques plus efficients et plus rapides.

\section{Technologique}

- L'évolution technologique rapide permet d'adopter les solutions les plus modernes pour la gouvernance publique, mais pour cela, le gouvernement doit accorder la priorité à d'autres domaines clés comme la cybersécurité, l'infrastructure d'authentification 
numérique, et améliorer les compétences des fonctionnaires en technologies de l'information et de la communication (TIC);

- L'interopérabilité entre les fichiers et les différentes institutions doit être garantie légalement et techniquement afin de pouvoir bénéficier des solutions numériques ;

- Il existe un besoin croissant de renforcer l'approche « expérience de l'utilisateur ». Ceci suppose de développer un nouvel ensemble de compétences et savoir-faire dans l'administration publique, pour permettre aux processus opérationnels d'être remaniés à la fois dans les front et back offices afin de fournir des services plus simples et conviviaux.

Légal

- La réglementation de la fonction publique est obsolète et n'encourage pas la mise en place d'un service public fondé sur le mérite, condition préalable pour une efficience accrue et une meilleure prestation de services ;

- L'abondance de la réglementations encadrant la prestation de services administratifs rend difficile la bonne compréhension des règles et leur parfaite application lors de la fourniture de ces services.

L'analyse SWOT est l'un des outils les plus populaires pour analyser l'environnement externe et interne de la politique concernée. Il s'agit d'une technique rapide et simple qui peut être utilisée pour étayer la préparation ou la révision des politiques, et elle suit souvent une analyse PESTEL. Les forces et les faiblesses ( $\mathrm{S}$ et $\mathrm{W}$ ) représentent les facteurs internes (par exemple, les moyens des organismes chargés de mettre en œuvre la politique, les aspects financiers et juridiques), tandis que les opportunités et menaces $(\mathrm{O}$ et $\mathrm{T})$ décrivent les catalyseurs et les freins (par exemple, la juste perception des bénéficiaires, les normes sociales, la démographie et l'économie).

L'analyse SWOT est menée au cours de sessions de réflexion, d'ateliers de travail et de groupes de discussion impliquant une grande variété de parties prenantes et des représentants d'organismes analogues. Dans le cas d'un exercice de SWOT lié à une RAP, il peut être utile d'obtenir les avis des usagers des services, des ONGs, d'universitaires, de praticiens et de décideurs politiques, en plus de ceux provenant des représentants d'organismes chargés de la mise en œuvre. Pour que l'analyse SWOT soit réussie, il sera nécessaire de la préparer en amont (avec par exemple, des questions permettant la participation des discussions préalables avec les parties prenantes et des lectures sur le sujet). Les sessions de SWOT sont organisées de manière à obtenir des informations pour chaque section de l'analyse, et les résultats sont présentés dans une matrice SWOT.

Le plus grand écueil d'une analyse SWOT est la confusion entre ce qui devrait être considéré comme une force ou une opportunité, une faiblesse ou une menace. Ce classement devrait toujours être le résultat d'une discussion entre les parties prenantes. Il faut noter qu'aucun facteur ne devrait apparaître sous plus d'une catégorie. 


\section{Encadré 6. Exemple de matrice SWOT théorique (exemple de RAP)}

\section{S}

- Les règles générales des procédures administratives garantissent des normes de démocratie et de fourniture équitable des services établies par la Constitution et au niveau international

- Grande expérience des institutions et individus dans le développement du gouvernement électronique dans l'administration publique, existence de services numériques de grande qualité dans certains domaines particuliers

- Existence d'une infrastructure TIC du gouvernement et d'un portail national de données ouvertes

\section{W}

- Faible rapport entre les résultats professionnels et la rémunération des employés

- Possibilités limitées pour une gestion flexible du personnel

- Organisation et coordination inefficace des TIC dans l'administration publique, les documents stratégiques ne sont pas liés et abordent insuffisamment, et de manière différente, le potentiel disponible des concepts numériques avancés

- Peu de transfert de connaissances et de bonnes pratiques liées à la numérisation

\section{O}

- Développement rapide dans la société d'un mode de vie numérique et mobile, permettant la mise en place accélérée du concept de gouvernement numérique

- Possibilité de plus nombreuses réformes de l'administration publique grâce à un cadre économique à moyen terme crédible

- Meilleure sensibilisation de la société aux questions de corruption

\section{$\mathbf{T}$}

- Faible niveau actuel d'utilisation des services électroniques, faible couverture des usagers finaux (citoyens et foyers) par l'internet haut débit, compétences numériques insuffisantes

- Persistance d'une couverture faible et inégale de l'accès à l'internet haut débit, qui peut ralentir la numérisation des services

La méthode de l'arbre à problèmes permet d'analyser d'une situation existante par l'identification des problèmes majeurs et de leurs principaux rapports de causalité. Le résultat de l'analyse se présente sous la forme d'une représentation graphique des problèmes, de leurs causes (raisons expliquant le problème) et de leurs effets (conséquences du problème).

La construction d'un arbre à problèmes est un exercice interactif qui implique des sessions de réflexion pour identifier les problèmes et leurs rapports de causalité. Il est recommandé d'inviter les principales parties prenantes (provenant des membres de la société civile, de l'université, du secteur privé ou d'autres institutions gouvernementales) à participer à l'exercice, afin que l'arbre à problèmes représente une vision consensuelle et partagée de la situation. Il peut être nécessaire d'avoir un facilitateur expérimenté pour mener avec succès une analyse par arbre à problème. La 
première tâche est d'identifier les problèmes centraux, puis les principaux rapports de causalité entre ceux-ci sont représentés dans l'arbre à problèmes.

Schéma 2. Exemple d'une analyse théorique par arbre à problème

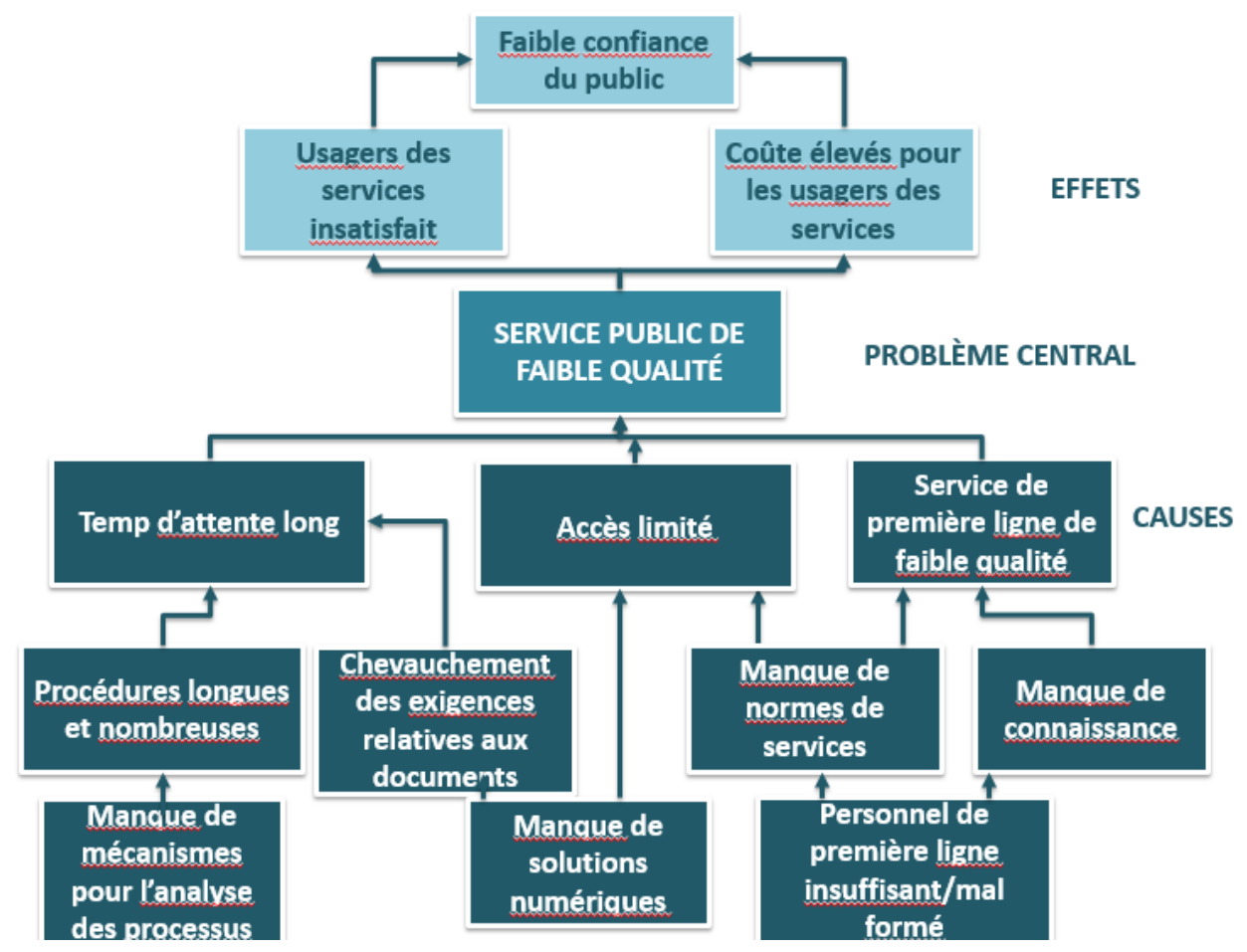

\subsection{Méthodes de recueil de données pour l'analyse}

Plusieurs méthodes de recueil de données peuvent être utilisées pour recueillir des informations et données sur la situation existante. Les méthodes les plus appropriées et les plus couramment utilisées pour l'analyse de l'état actuel de la situation comprennent :

- une recherche documentaire (suivi, évaluation ou autres rapports, examen réglementaire, utilisation des statistiques nationales et des données administratives, etc.)

- du brainstorming

- des groupes de discussion

- des entretiens

- des questionnaires ciblés

- des enquêtes et sondages d'opinion.

Le plus souvent, la bonne application de l'un des outils analytiques décrits dans la section 1.2 exige l'utilisation d'un mélange de plusieurs méthodes de recueil de données. Cela permet d'avoir une approche méthodologique plus solide et peut conduire à établir une base de conclusions plus fiable et plus robuste, et à parvenir à une compréhension commune des problèmes entre les parties prenantes impliquées. 
Lorsque différentes méthodes donnent des résultats ou des conclusions contradictoires (par exemple, si les données statistiques ou de mise en œuvre recueillies montrent que tout va bien, mais que des sources ou parties prenantes externes signalent des problèmes spécifiques pendant les entretiens), il est nécessaire d'approfondir la question, soit par la collecte de données additionnelles, soit en organisant des consultations supplémentaires ou des ateliers de collecte d'informations, afin de mieux comprendre les problèmes.

Il convient de rappeler que, dans de nombreux cas, il existe une stratégie précédant celle qui est en cours d'élaboration, et donc que le niveau d'ambition de la nouvelle stratégie doit être établi en faisant des références claires aux résultats de la stratégie précédente. Lorsque des rapports de suivi ou d'évaluation sont disponibles, leurs résultats et conclusions devraient avoir été utilisés au cours de l'analyse. Bien sûr, si les conclusions montrent que la stratégie précédente était de piètre qualité, ou impossible à mettre en œuvre, elle ne devrait être utilisée que de manière très restreinte lors de l'établissement des nouvelles ambitions stratégiques.

\subsection{Comment définir le cercle des institutions impliquées pour l'analyse}

Suivant l'étendue de l'analyse et les approches analytiques, outils et méthodes de recueil de données retenus, l'équipe chargée de l'analyse devrait toucher le plus large ensemble possible d'institutions, afin de garantir la solidité et la crédibilité de l'analyse. Reconnaître que même l'équipe interne la plus pointue ne peut posséder toutes les données et informations utiles à la richesse l'analyse, est indispensable pour comprendre quelles données supplémentaires devraient être recherchées et auprès de quelles sources, pour améliorer la compréhension de la situation existante et des qui nécessitent d'être résolus.

Lors de la détermination du cercle des institutions et parties prenantes qui seront impliquées dans l'analyse comme sources d'information, les responsables du processus analytique doivent en outre clairement définir ce qui est attendu des participants au processus, et le leur faire savoir. Ces attentes peuvent notamment comprendre :

- La fourniture de statistiques, données et informations qui ne sont pas accessibles à l'équipe chargée de l'analyse ;

- La fourniture d'avis (chaque avis formulé étant étayé par des preuves et des explications) ;

- La participation à des discussions liées à l'analyse de la situation et des problèmes, ou à la vérification des conclusions de l'analyse (par des réunions, des entretiens particuliers, des questionnaires écrits ou toute combinaison de ces méthodes);

- La clarification des informations (en particulier si différentes sources mènent à des conclusions contradictoires).

Le groupe d'institutions et de parties prenantes impliquées dans le processus analytique en tant que fournisseurs de renseignements et d'avis, et vérificateurs des conclusions, devrait être large, afin de permettre de recueillir le plus d'informations possibles sur le maximum possible d'aspects pertinents. Ceci devrait permettre d'éviter (ou du moins de limiter) toute partialité ou altération de l'analyse. Il faut bien sûr appliquer une proportionnalité des sources d'information et des institutions invitées à participer au recueil d'informations, conformément à la disponibilité des informations existantes et à la complexité de la question qui doit être analysée. Par ailleurs, l'équipe chargée de l'analyse doit rester concentrée pour mener l'intégralité du processus de recueil, 
d'analyse et de vérification d'informations en appliquant uniformément les méthodes retenues et en guidant les institutions et parties prenantes impliquées, afin de réussir à avoir, en fin de processus, une analyse cohérente, fiable et solide.

\section{Réflexions pratiques :}

$\checkmark$ Le plus important est de commencer à mener l'analyse!

$\checkmark$ Une analyse en interne est souvent rejetée car on suppose qu'elle exigera l'utilisation d'outils sophistiqués, un vaste recueil de données et de solides compétences analytiques qui ne sont pas disponibles en interne. Cette hypothèse n'est pas toujours correcte: l'analyse peut démarrer par une simple liste des problèmes et de leurs causes, puis être approfondie et enrichie par le " brainstorming ${ }^{28}$ (rassembler des parties prenantes pour discuter des problèmes et pour rechercher ensemble des solutions).

$\checkmark$ L'analyse en interne est utile car elle permet une meilleure vision interne des problèmes pressants et améliore les compétences et le savoir-faire des fonctionnaires.

$\checkmark$ Utilisez une large variété de sources d'information pour analyser les problèmes et produire des conclusions. Il peut s'agir des statistiques nationales, des données administratives, des rapports rendant compte de l'activité, des rapports d'évaluation, des rapports d'organisations européennes ou internationales (en particulier rapports CE et SIGMA), ainsi que des entretiens avec des représentants des principales institutions, partenaires du développement, ONGs ou autres parties prenantes externes.

$\checkmark$ Il y a toujours un équilibre délicat entre la sophistication de l'analyse, les ressources (et moyens) disponibles et les besoins réels. Une fois que les méthodes d'analyse et de recueil des données ont été établies, leur économie doit également être évaluée. Il ne sert à rien de dépenser du temps et de l'argent à obtenir des données très détaillées et complexes si cela n'est pas nécessaire pour le niveau de l'analyse requise.

$\checkmark$ Soyez pratique, pas scientifique, mais visez une analyse fiable, objective et proportionnée.

Ne pas oublier :

$\checkmark$ Planifiez toujours l'analyse en avance, et notamment la manière dont les résultats de l'analyse seront communiqués à l'intérieur et à l'extérieur de l'administration.

${ }^{28}$ Le brainstorming est utilisé dans le domaine des politiques publiques en faisant participer une assemblée de diverses parties prenantes à des discussions sur les principaux problèmes et questions, pour y trouver des solutions innovantes. Le principal objectif du brainstorming est d'obtenir une grande variété de perspectives, de la part de différentes parties prenantes, sur les questions en jeu. 
L'analyse doit mener à l'identification des problèmes centraux et de leurs causes profondes, et doit être étayée par des éléments probants.

$\checkmark$ L'analyse peut impliquer plusieurs méthodes de recueil d'informations, de mise en contexte et de recueil de données. Essayez d'utiliser différentes méthodes et des données fiables.

$\checkmark$ Les consultations sont importantes pour valider les résultats de l'analyse, ainsi que pour recueillir des données, informations et avis supplémentaires.

$\checkmark$ L'analyse peut être menée en interne, être externalisée, ou réalisée en combinant les deux démarches.

$\checkmark$ L'analyse peut prendre entre deux et six mois, voire plus.

$\checkmark$ Utilisez une large sélection de sources de données et d'informations (notamment des analyses externes récentes telles que les rapports d'organisations internationales comme l'OCDE ou la Banque mondiale, ou les évaluations SIGMA et les rapports par pays de la $C E$ ). 


\section{Cadrage et hiérarchisation}

L'information recueillie par l'analyse de problèmes - la liste des principaux problèmes et de leurs causes, étayées par des éléments probants - est utilisée pour établir des objectifs stratégiques. L'expérience montre cependant que les pays essaient souvent de traiter trop de problèmes dans leurs documents stratégiques, mettant en péril la réalisation des objectifs établis. Éviter ce risque nécessite soit de réduire la liste des objectifs et activités devrait soit être limitée (lorsque les ressources sont limitées), soit de l'échelonnée (lorsque certains problèmes exigent une action immédiate, tandis que d'autres sont moins urgents). Le cadrage et la hiérarchisation permettent d'y parvenir.

Définir la portée exacte de la stratégie et hiérarchiser les problèmes selon leur importance et leur urgence relatives revient à savoir dire "non». Une initiative stratégique ayant une portée quelque peu limitée mais un réel objectif et des plans d'intervention réalistes vaut bien plus qu'une stratégie au cadre large dont la mise en œuvre n'est pas réaliste.

En ce qui concerne la RAP, le cadrage (et la hiérarchisation) peut amener à limiter les objectifs concernant (1) les domaines de la RAP couverts et (2) les différents aspects d'un secteur donné de la RAP. Par exemple, certains domaines de la RAP peuvent être exclus s'ils ne présentent pas de problèmes particuliers, ou, en revanche, certains domaines peuvent être sélectionnés pour être traités en profondeur par la stratégie actuelle, même si cela signifie de laisser de côté pour le moment certains autres domaines aussi importants de la RAP. De la même manière, certains aspects de la fonction publique, par exemple, comme la rémunération ou le recrutement, peuvent être exclus de la stratégie s'ils ne présentent pas de problèmes majeurs. L'échelonnement sera reflété dans les objectifs et dans le calendrier des principales actions à entreprendre.

Si la portée et les priorités des réformes peuvent aussi être élaborées et décidées en interne, il est fortement recommandé de s'en acquitter en partenariat avec une large variété de parties prenantes, afin de valider les idées initiales de la réforme stratégique (et donc la sélection d'actions ciblées) développées par les principaux rédacteurs de la stratégie, et de garantir que les réformes sont élaborées de telle manière qu'elles reçoivent le plus large soutien possible de la part des principales parties prenantes ainsi que du public. Ainsi, il est plus probable que la sélection des problèmes devant être traités par les réformes, ainsi que les raisons ayant motivés ce choix soient acceptées par tous. Plus grand sera le consensus autour du sens des réformes, plus le pays aura de chances de les mener grâce aux efforts conjoints de toutes les parties prenantes impliquées dans leur mise en œuvre.

La clé du succès de l'exercice de cadrage et de hiérarchisation réside dans une discussion bien organisée et participative entre les hauts fonctionnaires (ceux qui détiennent un certain niveau de pourvoir de décision), les fonctionnaires et, dans certains cas, des parties prenantes externes. Des représentants de toutes les principales institutions chargées de mettre en œuvre la réforme devraient participer à la discussion, sinon la validité des résultats de la hiérarchisation - un accord sur un ensemble d'objectifs et sur les actions permettant de les réaliser - pourrait être remise en question, et l'appropriation pendant la mise en œuvre des réformes peut en souffrir. De plus, la discussion devrait être bien préparée, utilisant des documents de grande qualité exposant les résultats de l'analyse des problèmes et de premières suggestions quant aux questions stratégiques qui devraient être traitées par les réformes. La discussion devrait également être correctement modérée afin que tous les participants aient la possibilité de 
s'exprimer, et qu'à la fin du débat, un accord puisse être trouvé sur les principaux objectifs de la réforme et sur les actions à planifier pour atteindre ces objectifs. Les principaux intrants et résultats du processus de cadrage et de hiérarchisation sont donc :

- Intrant : documents bien préparés et discussion correctement modérée

- Résultat : au minimum, objectifs faisant l'objet d'un accord et (large) plan d'action pour atteindre ces objectifs. En outre, les indicateurs clés et des différentes étapes, et leurs cibles, ainsi que des précisions sur les responsables des diverses actions et des livrables à produire, peuvent être discutés et faire l'objet d'un accord, si le temps alloué à la (aux) discussion(s) commune(s) le permet.

Si plusieurs RAP ou stratégies sectorielles sont élaborées, il est recommandé de mener un exercice distinct de cadrage et hiérarchisation pour chacune d'entre elles.

Alors qu'une discussion participative pour fixer les priorités est le meilleur moyen d'arriver à un accord et de faire une sélection bien ciblée parmi les questions les plus pressantes nécessitent une réforme, la décision finale sur son étendue et ses priorités doit venir des décideurs de plus haut niveau responsables de l'adoption de la stratégie. Il peut donc être nécessaire de leur demander une décision préliminaire sur l'étendue et les priorités de la réforme envisagée, après qu'une discussion conjointe a eu lieu. Cependant, il est de la responsabilité partagée de tous les participants à de telles décisions de s'assurer que la réforme choisie est réaliste en termes de portée et de priorité, et que l'ensemble proposé d'objectifs retenus n'est pas irréaliste par rapport aux capacités de mise en œuvre et aux ressources disponibles du pays. Ce n'est que par un tel processus de sélection minutieux que la crédibilité de la stratégie peut être garantie.

\subsection{Outil de priorisation SIGMA pour l'élaboration d'une stratégie de RAP}

SIGMA a mis au point un outil de cadrage et de hiérarchisation pour faciliter le processus de hiérarchisation. Cet outil a été testé pendant l'élaboration des documents stratégiques exhaustifs de RAP de plusieurs partenaires (par exemple l'Albanie, la Géorgie, le Kosovo, et la Bosnie-Herzégovine). L'outil de hiérarchisation est fondé sur l'utilisation d'un simple questionnaire d'autoévaluation qui couvre tous les domaines de l'administration publique relevant du champ des Principes. L'Annexe 1 présente une explication détaillée de l'Outil de hiérarchisation.

L'utilisation de l'Outil de hiérarchisation dans l'élaboration d'une stratégie de RAP est facultative et dépend de la décision du pays. Cependant, cet Outil présente un avantage car il aide les développeurs de la stratégie en fournissant un modèle facile à utiliser, qui peut remplacer l'analyse de problèmes telle que décrite dans la Section I ci-dessus, puisqu'il exige de faire une brève évaluation de chaque Principe. De cette manière, l'Outil fournit un cadre d'évaluation logique pour chaque domaine et sous-domaine de l'administration publique, suivant la logique des Principes.

L'Outil de hiérarchisation comprend un questionnaire qui est conçu de façon à permettre aux diverses parties prenantes d'évaluer chaque aspect de l'administration publique du pays en utilisant une simple échelle numérique. Il fournit également une description des principales caractéristiques de la situation existante et comprend des suggestions sur la manière de les traiter. Le questionnaire couvre tous les Principes, mais il peut également être modifié en enlevant des éléments d'autoévaluation qui ne sont pas pertinentes dans le contexte particulier d'un pays (où, par exemple, certains domaines sont déjà couverts par des stratégies existantes), ou en ajoutant des éléments 
supplémentaires (par exemple lorsqu'un aspect particulier de l'administration publique requiert une évaluation et un ciblage particuliers et approfondis).

Il est important de sélectionner les personnes répondant au questionnaire de façon soigneuse mais exhaustive, afin de garantir que les contributions pertinentes sont recueillies auprès de l'ensemble des différentes parties prenantes responsables de, ou impliquées dans, les différents domaines couverts par le questionnaire (y compris, si possible, des informations provenant de parties prenantes externes). Il est également important que les personnes interrogées (notamment si elles sont censées représenter une réponse institutionnelle aux questions) comprennent pleinement le but du questionnaire, et soient expressément mandatées pour répondre, avec une consultation interne sur les réponses proposées ayant eu lieu avant qu'elles ne soient soumises (si nécessaire). C'est pourquoi les destinataires du questionnaire devraient être des responsables ou des décideurs institutionnels de haut niveau.

Il est également important de demander aux personnes interrogées d'éviter d'autocensurer leurs réponses, afin d'obtenir des observations sincères sur les principaux défis et problèmes. Pour cette raison, le principal organisme chargé de la hiérarchisation devrait assurer les personnes interrogées que les réponses seront traitées avec soin, et que seules les réponses agrégées seront partagées avec un public plus large.

Au cours du travail avec l'Outil de hiérarchisation SIGMA, les résultats agrégés du questionnaire, ainsi que tout autre intrant comme les analyses externes (y compris les rapports d'évaluation SIGMA, le cas échéant) et les détails de toute analyse de problèmes précédemment développée, sont utilisés pour faciliter une discussion destinée à parvenir à des conclusions communes sur les défis identifiés, à définir des objectifs pour y faire face, à esquisser les actions à mener pour atteindre ces objectifs. Il est indispensable, lorsqu'est utilisé l'Outil de hiérarchisation, qu'une telle discussion soit véritablement participative, fournisse une tribune pour s'exprimer et débattre des opinions divergentes lorsqu'elles se présentent, et qu'elle implique toutes les parties prenantes incontournables ( $\mathrm{y}$ compris externes), et tout particulièrement les principales institutions chargées des différents aspects de l'administration publique du pays.

Les principales tâches de la discussion sur la hiérarchisation consistent à :

- Harmoniser et valider l'analyse des problèmes et clarifier les conclusions ou résultats contradictoires, sur la base des scores donnés aux éléments du questionnaire, ainsi que des avis divergents sur l'urgence relative des aspects considérés ;

- Trouver un accord sur la sélection des questions ou défis centraux et les plus pressants en évaluant leur importance, leur urgence et leur complexité, ainsi que les ressources disponibles pour les traiter;

- S'accorder sur un ensemble indicatif d'objectifs et un plan (large) d'actions (et d'activités), sur la base des principaux défis identifiés.

L'application de l'Outil de hiérarchisation devrait être coordonnée par l'institution chef de file de la RAP, et son utilisation devrait impliquer toutes les institutions chargées de sa mise en œuvre. Il est de la plus grande importance que tous les représentants ayant un pouvoir de décision prennent part à la discussion et aux ateliers organisés pour la hiérarchisation. Il faut bien noter que la hiérarchisation ne sera valide que si les représentants ayant un pouvoir de décision y participent. De plus, la hiérarchisation devrait être un processus qui laisse à toutes les institutions participantes une possibilité 
de s'exprimer et de présenter leur opinion, et qui offre l'occasion de trouver un accord commun sur l'ossature du cadre stratégique de la RAP.

Les principaux résultats de l'exercice de hiérarchisation sont :

- Un ensemble harmonisé de conclusions et de scores provenant de l'analyse de problèmes ;

- Un accord sur une liste d'objectifs indicatifs et un (large) plan d'action validés, c'est-à-dire «l'ossature » de la stratégie de RAP.

Les produits et les résultats de la hiérarchisation sont utilisés pour définir les objectifs et les actions de la stratégie de RAP en cours d'élaboration.

L'utilisation de l'Outil de hiérarchisation SIGMA implique que les étapes ci-dessous soient suivies dans un ordre linéaire.

Schéma 3. Principales étapes de l'application de l'Outil de priorisation SIGMA

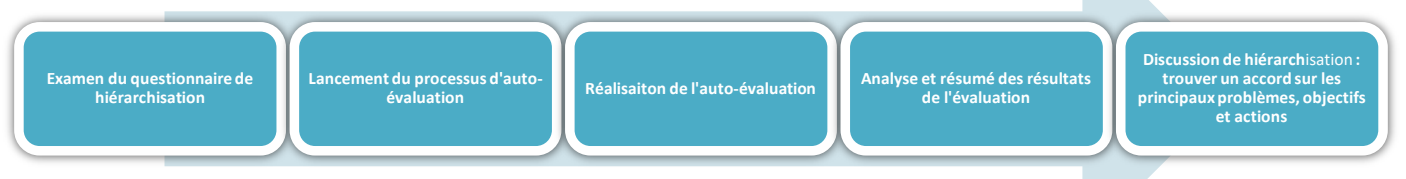

Réflexions pratiques :

$\checkmark$ La hiérarchisation est cruciale pour garantir que la réforme élaborée est réaliste et possible à mettre en œuvre.

$\checkmark$ Même si la hiérarchisation peut être réalisée par le petit cercle de personnes clés en charge de la (des) stratégie(s), il est préférable qu'elle soit le résultat d'une large consultation (idéalement équilibrée entre hommes et femmes), impliquant également des parties prenantes externes pour garantir que le choix des réformes ciblées à entreprendre est validée, et qu'augmentent le consentement à la réforme comme son appropriation.

$\checkmark$ La hiérarchisation participative économise du temps et des ressources lors des phases de mise en ouvre et de suivi en centrant les réformes sur les objectifs les plus pertinents, plutôt qu'en dispersant des ressources limitées dans des processus inutiles ou des initiatives de moindre importance ou valeur ajoutée.

$\checkmark$ Les priorités sélectionnées, ainsi que les raisons de leur sélection, devraient être largement communiquées afin d'assurer un soutien à la réforme, à la fois à l'intérieur et à l'extérieur de l'administration, et de démontrer l'engagement politique envers les réformes.

$\checkmark$ La hiérarchisation n'est pas un exercice sophistiqué exigeant l'utilisation d'outils complexes. Au contraire, elle peut ne demander qu'une légère autoévaluation de la situation et des problèmes, et est habituellement adaptée à l'expertise interne.

$\checkmark$ La hiérarchisation ne peut être réussie que si des décideurs de haut niveau sont impliqués et présents lors de la discussion, car celle-ci doit aboutir à des décisions 
importantes sur des questions importantes qui seront exclues de la réforme envisagée, étant donné le manque de ressources, qui doivent donc être allouées aux questions les plus urgentes.

\section{Ne pas oublier :}

Il est nécessaire de hiérarchiser les objectifs et les actions pour s'assurer que les réformes envisagées seront réalistes et possibles à mettre en œuvre. L'absence de hiérarchisation peut aboutir à l'élaboration d'une stratégie irréaliste et impossible à mettre en œuvre, avec pour conséquence une lassitude et une méfiance vis-à-vis des réformes.

$\checkmark$ Quelle que soit la méthode utilisée, il est de la plus grande importance d'avoir une discussion correctement modérée et inclusive sur les problèmes centraux et les principales actions.

$\checkmark$ Ce sont les décideurs qui doivent procéder à la hiérarchisation des sujets.

$\checkmark$ Lors de l'élaboration de stratégies de RAP, l'utilisation de l'Outil de hiérarchisation SIGMA est facultative. Son avantage est d'offrir un modèle facile à utiliser qui, une fois validé, fournit l'ossature d'un projet de stratégie de RAP qui peut être présentée aux principaux décideurs et discutée. 


\section{Fixation d'objectifs}

La vision, les objectifs, les indicateurs et les actions (interventions et activités) sont les éléments clés qui constituent les composantes de toute réforme. Ils suivent un ordre hiérarchique et servent à canaliser les ambitions politiques de la réforme et à les transformer en étapes ou initiatives réalisables.

Il est prouvé que, meilleure est la conception de la structure hiérarchique de la réforme, mieux elle peut servir à concentrer l'attention des dirigeants politiques et les efforts des institutions chargées de sa mise en œuvre pour créer un changement réussi et durable. Cette section explique certaines définitions et approches servant à définir une vision de la réforme, ainsi que la manière de traduire les défis définis et hiérarchisés en des objectifs généraux et spécifiques.

\subsection{Vision}

La vision consiste à envisager le futur désiré par rapport à l'état présent de l'administration publique en général ou de l'un un de ses secteurs. C'est le niveau le plus abstrait de la direction donnée à la réforme. La vision doit être encadrée dans le temps et assez ambitieuse pour inspirer et impulser les changements, mais doit tout de même être définie de manière à être réalisable.

La vision est habituellement définie par le petit groupe des principales parties prenantes centrales, puis partagée avec des groupes plus larges (idéalement équilibrés entre hommes et femmes) de parties prenantes. Les principales techniques utilisées pour définir la vision sont les méthodes interactives du brainstorming et de la table ronde. Dans le processus d'élaboration d'une stratégie, la vision est habituellement définie après l'achèvement de l'analyse des problèmes et lorsque la hiérarchisation a été effectuée. Cependant, elle peut aussi parfois être définie avant l'analyse, afin d'encourager une réflexion créative et progressive, orientée par la définition de la position conceptuelle finale à laquelle les décideurs veulent aboutir comme résultat des réformes. Dans ce cas, l'exposé de la vision peut être ajustée plus tard, si nécessaire, pour prendre pleinement en compte les résultats de l'analyse des problèmes. Quelle que soit la manière dont la vision est définie, il est important de créer un consensus autour de la nécessité et des objectifs de la réforme, notamment des raisons pour lesquelles le gouvernement veut engager un processus de réforme et quels objectifs fondamentaux il tente d'atteindre par les réformes.

L'exposé de la vision devrait être concis, court, rédigé clairement, facile à retenir, et devrait explicitement indiquer la direction à suivre. On considère que les exposés les meilleurs sont ceux qui font une ou deux phrases. S'il faut éviter de longs exposés, il n'est pas interdit de fournir quelques explications complémentaires pour étayer si nécessaire le projet de vision.

La vision n'est pas toujours exposée dans un document concernant une stratégie, et il peut être décidé de na pas la mentionner. Cependant, il peut être utile de matérialiser de manière simple les objectifs ultimes des réformes. L'encadré 7 en fournit quelques exemples. 
Encadré 7. Exemples de d'exposés de vision de RAP

- L'administration publique de (nom du pays) est conçue et fonctionne pour servir les citoyens de manière professionnelle et efficace, en garantissant les valeurs démocratiques et l'État de droit.

- L'administration publique de (nom du pays) est digne de confiance, transparente et $s$ 'acquitte efficacement de ses fonctions auprès des citoyens et entreprises.

- Les services publics sont fluides et efficients, et utilisent la meilleure technologie disponible pour garantir la satisfaction des citoyens et s'assurer de leur confiance.

- En 2020, l'administration publique de (nom du pays) est efficace, efficiente et responsable, sert les intérêts des citoyens, utilise les ressources financières de manière efficiente et applique des procédures transparentes.

\subsection{Objectifs stratégiques et leur hiérarchie}

Les objectifs sont les éléments desquels dépendra l'orientation des réformes applicables à certains défis connus, ils indiquent le sens des mesures à prendre et décrivent de manière tangible la situation future souhaitée. Ils sous-tendent toutes les actions, servent de base pour la création du cadre de la politique, et sont cruciaux pour le suivi et l'évaluation des performances.

Une stratégie peut avoir plusieurs niveaux d'objectifs. En principe, une stratégie devrait avoir deux niveaux d'objectifs : le niveau général et le niveau spécifique. Néanmoins, selon la portée de la stratégie et la complexité des défis auxquels elle est confrontée, et en conformité avec la logique de conception de la réforme dans son ensemble, les objectifs peuvent être soit plus agrégés soit davantage dissociés. Par exemple, la stratégie de RAP de la Bosnie-Herzégovine de $2006^{29}$ n'avait qu'un seul niveau d'objectifs, tandis que celles de la Serbie et du Monténégro en ont deux. La stratégie de RAP de l'Ukraine contient de priorités et des objectifs clés, la stratégie de RAP de la Géorgie a des objectifs généraux et des priorités, et la stratégie de RAP de la Moldavie a des objectifs généraux et spécifiques. Globalement, tout objectif, qu'il soit général ou plus spécifique, devrait être directement lié à des problèmes identifiés et à leurs causes, et aspirer à les résoudre. Il doit aussi servir de base à la définition d'indicateurs.

Un objectif général représente une aspiration plus générale et à long terme, sous la forme d'une déclaration sur l'amélioration de la situation que le gouvernement s'engage à atteindre en améliorant un domaine politique spécifique. Il couvre un domaine de réforme large mais bien défini et clairement établi, et sert donc à orienter une série d'actions de réformes destinées à parvenir à la situation future attendue.

En ce qui concerne la RAP, les objectifs généraux peuvent être reliés aux domaines couverts par les Principes d'administration publique, ou à un sous-ensemble d'éléments définis par les Principes dans l'un des domaines, ou dans plusieurs domaines. Utiliser les Principes pour définir les objectifs généraux permet aux pays de jeter les bases d'une administration efficace à travers un cadre conceptuel clair, fondé sur les bonnes pratiques internationales et approuvé par la CE. L'encadré 8 présente des exemples

29 Stratégie de réforme de l'administration publique de Bosnie-Herzégovine, 2006, http://parco.gov.ba//wp-content/uploads/2014/02/strategy-for-public-administration-reform.pdf 
d'objectifs généraux qui suivent les Principes. L'encadré 9 présente également de bons exemples d'objectifs généraux de RAP.

\begin{tabular}{|c|c|c|}
\hline \multicolumn{3}{|c|}{ Encadré 8. Exemples d'objectifs généraux suivant les Principes } \\
\hline Principes & Exemple 1 & Exemple 2 \\
\hline $\begin{array}{l}\text { Élaboration et } \\
\text { coordination des politiques }\end{array}$ & $\begin{array}{l}\text { Développer le processus } \\
\text { d'élaboration des politiques } \\
\text { en améliorant le système de } \\
\text { planification des mesures, } \\
\text { en renforçant le lien entre } \\
\text { planification des mesures et } \\
\text { budgétisation, et en } \\
\text { construisant un mécanisme } \\
\text { rigoureux de suivi, } \\
\text { d'évaluation et de compte } \\
\text { rendu d'activité. }\end{array}$ & $\begin{array}{l}\text { Renforcer la cohérence, } \\
\text { l'efficience, la prédictibilité } \\
\text { et la transparence des } \\
\text { processus de prise de } \\
\text { décision dans } \\
\text { l'administration publique. }\end{array}$ \\
\hline $\begin{array}{l}\text { Gestion des ressources } \\
\text { humaines et fonction } \\
\text { publique }\end{array}$ & $\begin{array}{l}\text { Création d'une fonction } \\
\text { publique efficace et } \\
\text { efficiente, basée sur un } \\
\text { système fondé sur le mérite } \\
\text { qui motive, promeut et } \\
\text { récompense le } \\
\text { professionnalisme. }\end{array}$ & $\begin{array}{l}\text { Adapter le système de } \\
\text { gestion des ressources } \\
\text { humaines aux objectifs et } \\
\text { aux exigences d'une } \\
\text { administration moderne. }\end{array}$ \\
\hline Responsabilité & $\begin{array}{l}\text { Améliorer l'intégrité et la } \\
\text { confiance du public, } \\
\text { accroître la transparence et } \\
\text { l'ouverture de } \\
\text { l'administration, la rendre } \\
\text { plus accessible et plus } \\
\text { responsable envers les } \\
\text { citoyens, et autonomiser } \\
\text { ceux-ci par l'utilisation des } \\
\text { nouvelles technologies et } \\
\text { par des approches modernes } \\
\text { et innovantes de la } \\
\text { gouvernance. }\end{array}$ & $\begin{array}{l}\text { Renforcer la responsabilité } \\
\text { des autorités } \\
\text { administratives, des } \\
\text { institutions publiques et des } \\
\text { entreprises publiques en } \\
\text { améliorant leur gestion. }\end{array}$ \\
\hline Prestation de services & $\begin{array}{l}\text { Réformer le système de } \\
\text { prestation de services pour } \\
\text { fournir des services de haute } \\
\text { qualité, accessibles et bien } \\
\text { gérés dans l'ensemble du } \\
\text { pays. }\end{array}$ & $\begin{array}{l}\text { Améliorer l'accès, } \\
\text { l'efficience et l'efficacité } \\
\text { des services publics aux } \\
\text { niveaux central et local en } \\
\text { réduisant les charges } \\
\text { administratives inutiles, en } \\
\text { abaissant les coûts et en } \\
\text { prenant en compte les } \\
\text { besoins de bénéficiaires. }\end{array}$ \\
\hline $\begin{array}{l}\text { Gestion des finances } \\
\text { publiques }\end{array}$ & $\begin{array}{l}\text { Garantir la stabilité et la } \\
\text { répartition efficace des }\end{array}$ & $\begin{array}{l}\text { Créer un système de gestion } \\
\text { financière moderne, sur des }\end{array}$ \\
\hline
\end{tabular}




\begin{tabular}{|c|c|c|}
\hline & $\begin{array}{l}\text { finances publiques en } \\
\text { conformité avec les priorités } \\
\text { du gouvernement. }\end{array}$ & $\begin{array}{l}\text { principes qui garantiront la } \\
\text { rigueur budgétaire et des } \\
\text { procédures transparentes } \\
\text { pour l'administration du } \\
\text { budget de l'État. }\end{array}$ \\
\hline
\end{tabular}

Un objectif spécifique indique des résultats plus concrets, et se concentre plus précisément sur un aspect d'un domaine (ou d'un ensemble de domaines) défini dans un objectif général, habituellement dans une perspective à court ou moyen terme. D'une part, les objectifs spécifiques sont définis pour donner des orientations plus précises aux actions, et chacun de ces objectifs doit être atteint par la mise en œuvre d'une ou plusieurs actions. D'autre part, ces objectifs doivent être liés à un objectif général et représenter un moyen d'atteindre celui-ci. Les objectifs spécifiques peuvent également être reliés aux prérequis de chaque secteur couvert par les Principes.

En règle générale, tous les objectifs (spécifiques ou généraux) devraient être harmonisés entre eux et contribuer ensemble à atteindre le niveau suivant d'objectifs, et, en fin de compte, la vision de la réforme.

\begin{tabular}{|c|c|c|c|}
\hline \multicolumn{4}{|c|}{ Encadré 9. Exemples d'objectifs généraux de RAP } \\
\hline $\begin{array}{l}\text { Objectif } \\
\text { général }\end{array}$ & $\begin{array}{l}\text { Établir un système } \\
\text { de fonction } \\
\text { publique fondée sur } \\
\text { le mérite et la } \\
\text { promotion d'une } \\
\text { gestion des } \\
\text { ressources } \\
\text { humaines efficace } \\
\text { pour favoriser une } \\
\text { prestation de } \\
\text { services plus } \\
\text { professionnelle }\end{array}$ & $\begin{array}{l}\text { Améliorer la qualité } \\
\text { des services publics } \\
\text { pour mieux répondre } \\
\text { aux besoins des } \\
\text { usagers }\end{array}$ & $\begin{array}{l}\text { Renforcer la } \\
\text { cohérence, l'efficience, } \\
\text { la prédictibilité et la } \\
\text { transparence des } \\
\text { processus de prise de } \\
\text { décision dans } \\
\text { l'administration } \\
\text { publique. }\end{array}$ \\
\hline $\begin{array}{l}\text { Objectif } \\
\text { spécifique } 1\end{array}$ & $\begin{array}{l}\text { Élargir la portée de la } \\
\text { fonction publique } \\
\text { pour couvrir tous les } \\
\text { postes dans le } \\
\text { système }\end{array}$ & $\begin{array}{l}\text { Améliorer l'accès } \\
\text { physique et numérique } \\
\text { aux services publics }\end{array}$ & $\begin{array}{l}\text { Créer de nouveaux } \\
\text { mécanismes (ou } \\
\text { renforcer les } \\
\text { mécanismes existant) } \\
\text { pour une politique } \\
\text { fondée sur des éléments } \\
\text { concrets et cohérente, } \\
\text { conforme au cadre légal }\end{array}$ \\
\hline $\begin{array}{l}\text { Objectif } \\
\text { spécifique } 2\end{array}$ & $\begin{array}{l}\text { Créer un système de } \\
\text { recrutement équitable }\end{array}$ & $\begin{array}{l}\text { Améliorer la prestation } \\
\text { des services publics par } \\
\text { la définition de normes }\end{array}$ & $\begin{array}{l}\text { Aligner les prévisions } \\
\text { politiques sur le cadre }\end{array}$ \\
\hline
\end{tabular}




\begin{tabular}{|c|c|c|c|}
\hline & $\begin{array}{l}\text { et fondé sur les } \\
\text { compétences }\end{array}$ & $\begin{array}{l}\text { de qualité pour les } \\
\text { services publics }\end{array}$ & $\begin{array}{l}\text { budgétaire à moyen } \\
\text { terme du budget annuel }\end{array}$ \\
\hline $\begin{array}{l}\text { Objectif } \\
\text { spécifique } 3\end{array}$ & $\begin{array}{l}\text { Créer un système de } \\
\text { rémunération fondé } \\
\text { sur les performances }\end{array}$ & $\begin{array}{l}\text { Sensibiliser les usagers } \\
\text { de service à l'accès } \\
\text { numérique }\end{array}$ & $\begin{array}{l}\text { Augmenter la } \\
\text { transparence des } \\
\text { politiques } \\
\text { gouvernementales en } \\
\text { assurant des } \\
\text { consultations publiques } \\
\text { et l'accès des citoyens } \\
\text { aux décisions }\end{array}$ \\
\hline $\begin{array}{l}\text { Objectif } \\
\text { spécifique } 4\end{array}$ & & & $\begin{array}{l}\text { Renforcer l'aptitude du } \\
\text { bureau gouvernemental } \\
\text { et du ministère des } \\
\text { Finances à accomplir } \\
\text { des fonctions de « centre } \\
\text { de gouvernement» }\end{array}$ \\
\hline
\end{tabular}

En règle générale, il ne devrait y avoir qu'un nombre limité d'objectifs, pour se concentrer et mobiliser les ressources sur la réalisation de ces objectifs. Des objectifs trop nombreux auront pour effet de fractionner des ressources limitées et peuvent aboutir à une exécution mal-ciblée, et donc sous-optimale, des politiques et réformes. Comme décrit ci-dessous, les objectifs devraient être directement liés aux problèmes identifiés et à leurs causes.

\subsection{Lier les objectifs aux problèmes et à leurs causes}

Les objectifs ne sont pas définis isolément. En premier lieu, ils doivent traiter des problèmes identifiés. En second lieu, ils doivent être étayés par des actions pertinentes et qui leur sont liées, et être traduits et explicités par des indicateurs pertinents. La condition préalable cruciale pour fixer un bon objectif est donc l'analyse des problèmes menant à l'identification des principaux défis et de leurs causes. Si l'analyse des problèmes est correctement menée et que les problèmes sont bien définis et hiérarchisés (sur ce sujet, voir la Section II), l'élaboration des objectifs ne devrait pas poser de difficulté.

On peut arriver à une définition initiale des objectifs en transformant les problèmes identifiés et leurs causes en propositions positives, comme il est montré dans le Tableau 1. Cela est simple, pourvu que les problèmes et leurs causes aient été suffisamment identifiés et définis, et que l'analyse reflète de façon réaliste les problèmes centraux les plus urgents. Très souvent, les problèmes et leurs causes sont compartimentés, et considérés à tort comme interchangeables. Si les problèmes centraux ne sont pas définis comme ils devraient l'être, mais subdivisés en sous-problèmes, il y a une grande chance que les objectifs soient également dispersés, ce qui aboutit à un cadre stratégique extrêmement complexe et une orientation sous-optimale pour la réforme. Globalement, si le travail d'identification des problèmes est bien fait, il devrait y avoir le même nombre d'objectifs que celui de problèmes et de causes, mais uniquement concentrés sur la résolution des problèmes majeurs. 
Tableau 1. Problèmes et Objectifs (exemple de RAP)

\begin{tabular}{|c|c|c|}
\hline Problèmes et leurs causes & & Objectifs généraux et spécifiques (en gras) et actions associées \\
\hline $\begin{array}{l}\text { Problème : Services publics de } \\
\text { mauvaise qualité }\end{array}$ & $\rightarrow$ & Améliorer la qualité des services publics \\
\hline Cause : Temps d'attente long & $\rightarrow$ & $\begin{array}{l}\text { Créer un mécanisme de réaménagement des procédures } \\
\text { administratives pour les services publics } \\
\text { Mener un réaménagement des procédures administratives pour dix } \\
\text { services sélectionnés }\end{array}$ \\
\hline Cause : Accès limité aux services & $\rightarrow$ & $\begin{array}{l}\text { Accroître la numérisation des services } \\
\text { Numériser dix services publics pour atteindre une interaction de niveau } \\
4 \\
\text { Élargir l'accès physique aux services dans les régions en utilisant } \\
\text { l'infrastructure publique actuelle } \\
\text { Créer des centres de services dans les bureaux de poste et les centres } \\
\text { sociaux }\end{array}$ \\
\hline $\begin{array}{l}\text { Cause : Service de première ligne } \\
\text { de mauvaise qualité }\end{array}$ & $\rightarrow$ & $\begin{array}{l}\text { Développer des normes de service } \\
\text { Former les prestataires de services conformément aux normes de } \\
\text { service }\end{array}$ \\
\hline $\begin{array}{l}\text { Problème : Structure de } \\
\text { l'administration } \\
\text { inappropriée }\end{array}$ & $\rightarrow$ & Moderniser la structure de l'administration publique centrale \\
\hline $\begin{array}{l}\text { Cause: Manque d'analyse des } \\
\text { fonctions des institutions de } \\
\text { l'administration publique }\end{array}$ & $\rightarrow$ & $\begin{array}{l}\text { Optimiser le nombre et les fonctions des autorités administratives } \\
\text { publiques } \\
\text { Établir un mécanisme d'examen régulier et cohérent de l'efficacité et } \\
\text { de l'efficience de la structure administrative } \\
\text { Entamer une analyse pour examiner les fonctions des autorités } \\
\text { administratives publiques }\end{array}$ \\
\hline
\end{tabular}

De cette manière, les objectifs sont directement liés aux problèmes et à leurs causes, identifiés lors de l'exercice d'analyse des problèmes et de hiérarchisation, et forment les éléments clés de la stratégie.

\subsection{Principaux aspects du processus de fixation d'objectifs}

Le processus de fixation d'objectifs devrait être participatif et approuvé par toutes les principales parties prenantes. Il peut prendre la forme d'un atelier de hiérarchisation, également utilisé pour élaborer les projets d'objectifs, ou celle d'une série de réunions ou ateliers conjoints, réunissant toutes les parties prenantes.

Les objectifs d'une stratégie devraient être aussi courts que possible, et formulés dans un langage simple. Leur formulation devrait éviter l'usage d'abréviations, de jargon professionnel et de phrases longues et complexes.

La pertinence des objectifs devrait être testée à l'aune du modèle «SMART». Les objectifs devraient être : 
- $S P E C I F I Q U E$ - un objectif doit être concret, décrire le résultat à atteindre, et centré, contribuant à la solution du problème ;

- MESURABLE - un objectif DOIT pouvoir être exprimé de manière numérique et quantitative par rapport à une référence spécifique, et permettre de suivre les progrès de la mise en œuvre ;

- AXÉ SUR L'ACTION/ACCEPTABLE/ATTEIGNABLE - un objectif doit motiver une action, et spécifier ce qui doit être amélioré, augmenté, renforcé, etc., mais il doit aussi être atteignable ;

- $\boldsymbol{R} E ́ A L I S T E$ - un objectif doit être réaliste en termes de délais et de ressources disponibles ;

- TEMPORELLEMENT DÉFINI - la réalisation de l'objectif doit s'inscrire dans une période donnée.

Ce sont souvent les indicateurs associés et leurs cibles et valeurs de référence qui rendent un objectif mesurable et l'inscrivent dans le temps. Il est néanmoins important de garder ce modèle en tête afin de fixer des objectifs simples, clairs et faciles à lire.

La fixation d'objectifs est un processus itératif, et il peut être nécessaire de réviser les objectifs plusieurs fois avant qu'ils ne soient finalisés. Ils peuvent être révisés à plusieurs occasions :

- Après avoir fixé les indicateurs et leurs valeurs de référence et les cibles correspondantes, ainsi que leur fiche d'identité détaillant la façon de les mesurer, car certains objectifs peuvent avoir été trop largement ou trop étroitement définis ;

- Après la planification et le chiffrage des actions, certains objectifs pouvant apparaître comme trop ambitieux et irréalisables étant donné les circonstances et les ressources disponibles ;

- Après qu'un examen des objectifs a révélé que leur formulation n'était pas suffisamment claire.

L'ensemble d'objectifs devrait « raconter l'histoire » de la stratégie de manière logique et séquentielle, par conséquent ils devraient être combinés logiquement. Ils devraient être en rapport avec l'ensemble des problèmes considérés comme nécessitant une réforme et, lorsque plusieurs niveaux d'objectifs sont utilisés, ils devraient être liés les uns aux autres, afin de fournir une image complète des réformes envisagées.

Aucun objectif ne devrait se rapporter à des réformes non justifiées par des problèmes identifiés au cours de l'analyse. Au contrario, aucun des problèmes identifiées ne devrait être omis par un objectif, ou plusieurs objectifs, à moins qu'il ne soit clairement expliqué pourquoi le problème en question n'est pas traité dans la stratégie, par exemple à la suite d'une hiérarchisation solide et incontestable. Tout comme les problèmes identifiés et les priorités retenues pour la réforme, les objectifs adoptés devraient eux aussi être correctement communiqués afin de s'assurer que des efforts concourront à leur réalisation et pour faire preuve de transparence, d'engagement et de responsabilité. 


\section{Réflexions pratiques :}

$\checkmark$ Les objectifs ne doivent pas être écrits comme une loi ou un règlement. Ils n'ont pas besoin de refléter un vocabulaire légal ou technique. Les meilleurs objectifs sont écrits en langage simple.

$\checkmark$ L'une des principales difficultés pour fixer des objectifs est leur dispersion. Lorsque l'ensemble initial d'objectifs est achevé, comptez toujours le nombre d'objectifs que vous avez pour un même domaine. Il ne faut pas oublier qu'il y a toujours moyen de fusionner et de rationaliser. Vérifiez toujours deux fois cet aspect!

\section{Ne pas oublier :}

$\checkmark$ Les objectifs stratégiques peuvent être de plusieurs niveaux, tels que général et spécifique.

$\checkmark$ Les objectifs stratégiques ne sont pas fixés isolément, ils visent à traiter des problèmes définis et sont logiquement reliés à des activités spécifiques, et leur réalisation est mesurée par des indicateurs.

$\checkmark$ Le nombre d'objectifs doit être limité afin de concentrer les ressources et l'attention sur les problèmes centraux.

$\checkmark$ Les objectifs doivent raconter l'histoire de la (des) réforme(s), en expliquant clairement ce qui sera changé, comment, et quand (l'utilisation d'indicateurs joue aussi un rôle crucial dans ce domaine - voir Section IV). 


\section{4. Élaboration d'indicateurs}

L'étape de construction des indicateurs, de leurs valeurs de référence et de leurs cibles, est cruciale dans le processus d'élaboration de la stratégie. Très souvent, les indicateurs, et plus spécialement leurs valeurs de référence et cibles sont négligés dans l'étape de l'élaboration; la stratégie reste à l'état de document vague qui n'indique pas convenablement aux personnes chargées de sa mise en ouvre ce qu'elles doivent réellement réaliser, et le public est laissé sans définition claire et identifiable des ambitions du gouvernement dans la réforme d'un secteur ou d'un domaine particulier de l'administration publique. Les indicateurs, leurs valeurs et leurs cibles sont utilisés pour mesurer tant le niveau de succès et les progrès accomplis en regard des objectifs stratégiques retenus, que les actions et activités concrètes ${ }^{30}$.

Le mot « indicateur » renvoie aux moyens par lesquels un objectif ou une action peut être ou non considéré comme ayant été atteint ou mis en œuvre ou non. C'est pourquoi les indicateurs sont directement liés aux objectifs et aux actions (y compris les activités ou « mesures ») contenus dans la stratégie. Ils servent de références à l'aune desquelles mesurer le degré de succès dans la réalisation d'un objectif, ou vérifier si une action a été mise en œuvre, et quel a été son résultat. Les indicateurs ne sont pas seulement des instruments de mesure de l'atteinte d'un objectif, en étant en lien avec les objectifs et actions stratégiques qu'ils cherchent à améliorer ils doivent aussi être exploitables : l'indicateur et sa cible doivent permettre de prendre les mesures ayant pour but d'améliorer la situation ou d'entreprendre des actions correctives si la situation évolue dans le mauvais sens ${ }^{31}$.

Les indicateurs sont des outils qualitatifs ou quantitatifs, et sont habituellement exprimés en taux, ration, pourcentage ou volume, ou de manière narrative (par exemple «la mesure dans laquelle...», «le degré de...»). Les indicateurs qualitatifs (par exemple, exprimant un jugement informé sur un aspect de la réforme), peuvent également, grâce à leurs cibles pré-établies, indiquer le sens du changement (une réduction ou une augmentation de quelque chose), mais un équilibre subtil doit être trouvé entre de tels indicateurs et des indicateurs de quantité. En fin de compte, ce sont les objectifs, actions et activités définis qui devraient dicter le type de mesure le mieux approprié pour refléter les progrès de la mise en œuvre, et la réalisation des ambitions de la réforme.

Aucun indicateur ne reflète l'intégralité d'un objectif de réforme. Ils fournissent plutôt des exemples donnant une explication concrète et comparative du progrès accompli au cours de la (des) réforme(s). Plus simplement, un indicateur exprime le progrès, le

${ }^{30}$ Pour plus d'information sur l'établissement des indicateurs, voir :

https://www.nao.org.uk/wp-content/uploads/2013/02/fabric.pdf; et OCDE (2009), Conduire les politiques de développement régional : Les indicateurs de performance, Éditions OCDE, Paris, https://doi.org/10.1787/9789264058385-fr et Commission européenne (2016), Guidelines on linking planning/programming, monitoring and evaluation, Bruxelles https://ec.europa.eu/neighbourhoodenlargement/sites/near/files/pdf/financial_assistance/phare/evaluation/2016/20160831-dg-nearguidelines-on-linking-planning-progrming-vol-1-v-0.4.pdf

${ }^{31}$ Howell, Marwin T. (2006), Actionable Performance Measurement: A Key to Success, ASQ Quality Press, Milwaukee, Wisconsin. 
succès ou l'échec à peu près de la même manière qu'un thermomètre mesure la température, de froid à chaud, à brûlant !

Les indicateurs sont utiles pour différents groupes :

- Les responsables et personnels de ministères/d'institutions ont besoin de savoir si les ressources affectées à la mise en œuvre d'une stratégie sont utilisées de manière efficace, et si la combinaison d'activités mène aux résultats souhaités.

- Le gouvernement doit comprendre si une stratégie adoptée aboutit aux résultats souhaités, et si les dépenses publiques sont judicieusement investies.

- Les usagers de services et le public dans son ensemble doivent savoir si la performance de l'administration publique (et, par conséquent, la qualité des services), s'améliore.

Afin de pouvoir mesurer les progrès, les indicateurs doivent avoir une valeur de référence et définir une valeur cible. La valeur de référence reflète la situation juste avant ou au commencement d'une nouvelle stratégie, par rapport à laquelle le progrès sera mesuré ou des comparaisons seront faites, dans le cadre du processus de suivi et d'évaluation. Dans la plupart des cas, la référence est le niveau de performance enregistré dans l'année ou la période précédente. Une valeur cible est celle que l'indicateur est censé atteindre à un moment déterminé. Un indicateur peut avoir différentes valeurs cibles intermédiaires (ou "valeurs d'étape ») qui permettent de mesurer le degré de réalisation plusieurs fois au cours de la mise en œuvre de la réforme ${ }^{32}$.

\subsection{Types d'indicateurs}

Si de nombreux gouvernements mesurent les performances, il existe des différences dans leur façon de classifier et d'utiliser les indicateurs. Les types d'indicateurs les plus fréquemment utilisés sont présentés dans le Tableau 2.

\footnotetext{
${ }^{32}$ Les indicateurs doivent être élaborés de manière à ce qu'on puisse identifier séparément, par leur mesure, les résultats des actions du gouvernement et les autres facteurs affectant le résultat final. Ceci peut être testé au mieux pendant l'évaluation par une " analyse contrefactuelle », lorsque l'impact de l'action gouvernementale peut être isolée et évaluée.
} 
Tableau 2. Types d'indicateurs

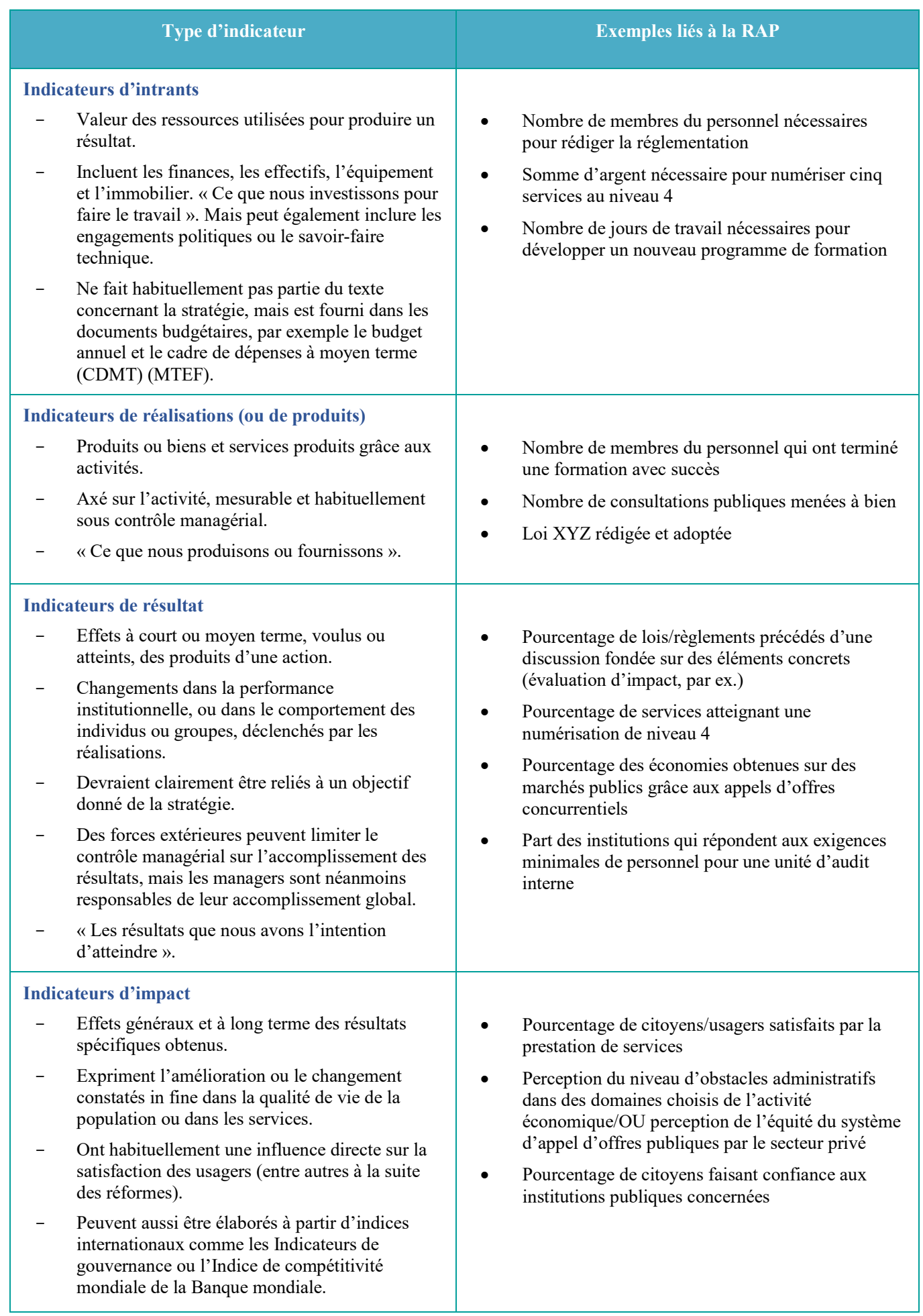

Note : L'utilisation d'indices internationaux dans la construction d'indicateurs devrait se faire avec une attention particulière, car ils sont complexes et difficiles à reconstruire. L'utilisation biunivoque de tels indices comme indicateurs devrait généralement être évitée, car les indicateurs devraient toujours être liés aux spécificités de la réforme élaborée. 
Les indicateurs ont également une hiérarchie logique (ou « cadre logique »), comme le montrent les Schémas 4 et 5 , qui représentent ce cadre et présentent les liens et les questions clés liées aux différents types d'indicateurs. Un intrant est toujours externe à ce qui a été directement produit (le produit). La production ou réalisation de certains produits se traduit par un changement, qui est à son tour décrit comme un résultat particulier. L'obtention de certains résultats peut induire un changement collectif dans l'environnement global des parties prenantes, appelé impact ${ }^{33}$. L'identification correcte du type d'indicateur signifie aussi qu'il est correctement positionné dans cette hiérarchie ${ }^{34}$. La meilleure méthode pour y parvenir est de poser les questions clés pour chaque indicateur: mesure-t-il la production d'un produit ou d'un service, décrit-il l'obtention des résultats du changement prévu, montre-t-il un changement réel dans des usagers finaux des services de l'administration publique, etc. (Voir Schéma 4).

\section{Schéma 4. Types d'indicateurs}

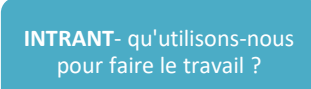

${ }^{33}$ L'impact intégral peut être évalué par la méthode/analyse contrefactuelle, lorsque la question porte sur ce qui se serait passé si l'intervention n'avait pas eu lieu. C'est habituellement une question clé de l'évaluation.

34 Des typologies différentes de celle qui est présentée ici existent dans la littérature internationale. Par exemple, les indicateurs de processus sont également largement utilisés. Ils mesurent le processus (ou transformation) des intrants en produits. 
Schéma 5. Exemple d'un cadre logique de RAP mettant en évidence les liens entre objectifs et indicateurs

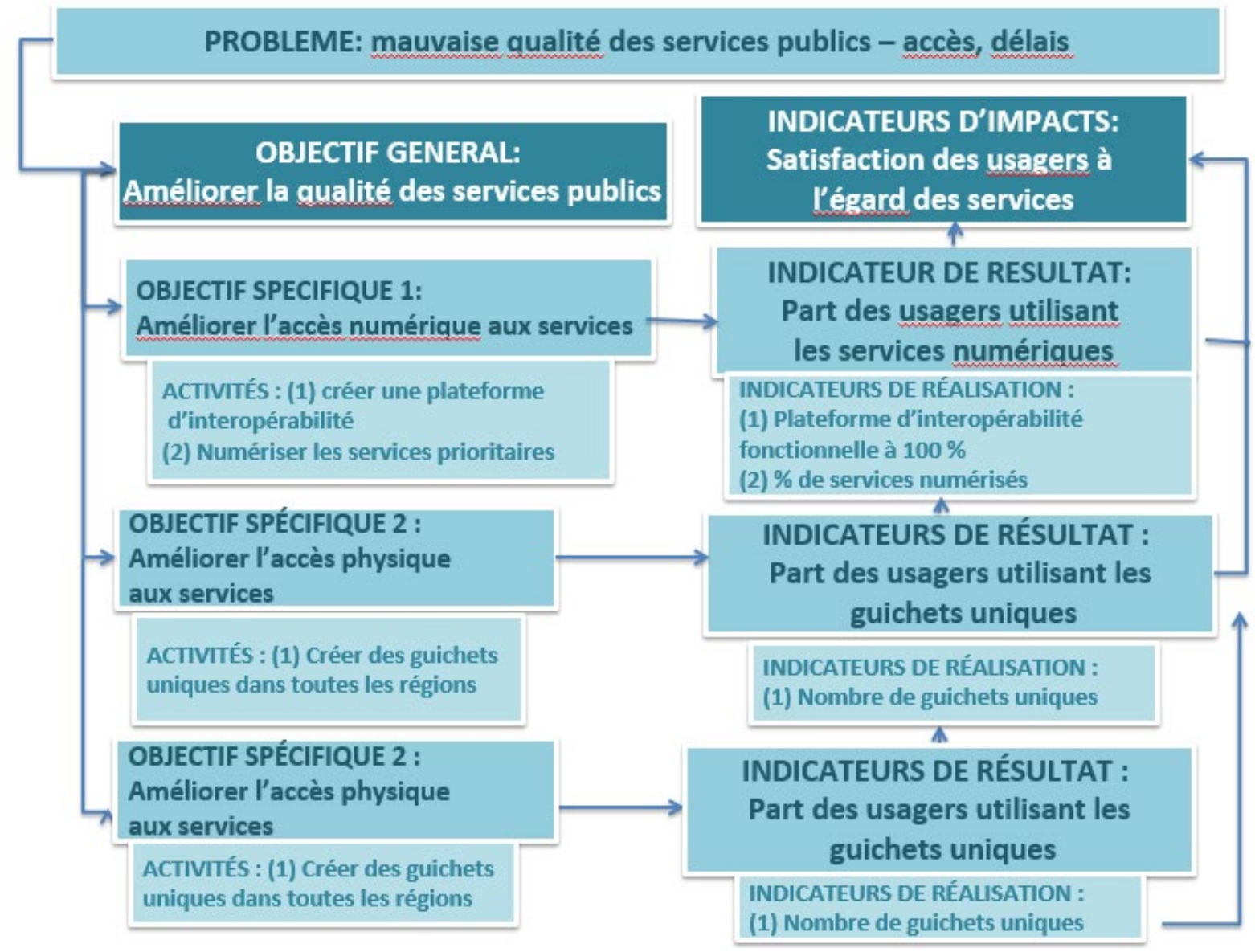

4.2. Les liens entre objectifs et actions et les indicateurs mesurant leur réalisation

Au cours de l'élaboration de la stratégie, il est important d'établir une liaison directe entre les indicateurs, et les objectifs et actions qu'ils doivent mesurer. Les objectifs seront réalistes et atteignables s'ils sont logiquement reliés à d'autres éléments de la stratégie :

1. Les problèmes identifiés sont résolus à travers des objectifs généraux, qui doivent être directement liés aux problèmes identifiés et à leurs causes. Un objectif général est habituellement mesuré par un indicateur de niveau d'impact, bien qu'il puisse parfois être seulement mesuré par un indicateur de niveau de résultat.

2. Les objectifs généraux doivent être mis en œuvre par un ensemble d'objectifs spécifiques. Pour chaque objectif spécifique, il doit y avoir un (des) indicateur(s) de niveau de résultat.

3. Les objectifs spécifiques seront (notamment) atteints par la mise en œuvre d'un nombre limité d'actions (activités, programmes, mesures, etc.). Chacune de ces actions doit avoir un budget et un (des) indicateur(s) de niveau de réalisation montrant ce qui a été réalisé par l'action.

4. Les indicateurs et les informations de niveau d'intrant se trouvent dans les documents rattachés au budget tels que les programmes budgétaires ou le budget 
annuel, ainsi que dans les documents d'appui au plan d'action de la stratégie de RAP ou dans d'autres sources de données administratives telles que les données détaillées sur l'emploi ou les salaires, ainsi que dans les statistiques nationales.

Dans l'idéal, une stratégie devrait avoir autant de niveaux d'indicateurs que de niveaux d'objectifs. De plus, les actions (les activités spécifiques retenues) devraient également avoir des indicateurs concrets de niveau de réalisation. Par défaut, une stratégie devrait comporter des indicateurs de niveau de réalisation et de niveau de résultat pour mesurer l'atteinte des objectifs prédéterminés et la mise en œuvre des actions destinées à atteindre ces objectifs. Les indicateurs de niveau d'impact peuvent (et, idéalement, devraient) être également utilisés pour régulièrement mesurer l'impact global des réformes sur l'environnement qu'elles visent à transformer. La meilleure manière d'évaluer les réformes est de mesurer l'impact par la méthode contrefactuelle, en s'interrogeant sur ce qui se serait passé sans les actions de réforme.

Il est recommandé de n'utiliser qu'un nombre limité d'indicateurs, afin de pouvoir gérer la charge de travail et les coûts du suivi, car il s'agit d'un processus exhaustif et exigeant (voir Section VII), qui peut souvent exiger un recueil supplémentaire de données, parfois auprès de sources primaires telles que des enquêtes conçues spécifiquement pour le suivi de la mise en œuvre de la stratégie. Par ailleurs, il est nécessaire de cerner les aspects centraux de tous les objectifs afin d'être en mesure de suivre intégralement les progrès par rapport à ce qui était prévu par les objectifs. Il est recommandé d'avoir un à trois indicateurs (d'impact ou de résultat) par objectif, et au moins un indicateur (de réalisation) par action.

Il arrive très souvent que les objectifs retenus pour une stratégie ne figurent pas dans le plan d'action et n'aient pas d'indicateurs mesurables avec des références et des cibles. Si cette pratique peut aider à la lecture du document exposant la stratégie lui-même, elle peut entraver la construction et l'utilisation d'un cadre approprié de suivi permettant de vérifier régulièrement que les réformes envisagées sont réalisées. Si le plan d'action est séparé du reste de la stratégie, il est fortement recommandé de répéter tous les objectifs stratégiques définis (généraux comme spécifiques) dans le plan d'action et de les lier aux activités individuelles définies. Il est également important de s'assurer que chaque objectif général et spécifique est lié à au moins un indicateur mesurable.

\subsection{Désignation des personnes chargées de la mesure et de la qualité des indicateurs}

La définition des responsabilités institutionnelles est une question cruciale pour l'établissement des indicateurs, notamment pour la fixation de leur valeur de référence et de leur valeur cible. Il est important de spécifier les choses suivantes :

- titre et définition de l'indicateur,

- manière dont l'indicateur est calculé,

- qui est responsable du recueil de données pour chaque indicateur,

- la fréquence requise du recueil de données,

- qui est responsable des rapports sur les progrès relatifs à chaque indicateur, ses étapes (cibles intermédiaires), et ses cibles finales,

- qui est chargé de l'analyse des causes des bonnes ou mauvaises performances (c'est-à-dire de la partie analytique des rapports de performance). 
Il est nécessaire d'établir clairement ces responsabilités au préalable pour avoir un suivi efficace et dans des délais convenables, car cela permet à tous les responsables ayant des responsabilités attribuées de se préparer en avance et de faire en sorte que l'élaboration des rapports ne souffre aucun retard dû à une définition peu claire des responsabilités et du calendrier de mise à disposition des données. Il est recommandé que d'attribuer ces responsabilités dans les documents exposant la stratégie, par exemple dans une annexe détaillant les indicateurs choisis.

Il n'est pas facile d'établir une bonne liste d'indicateurs. Il est recommandé d'appliquer plusieurs critères lors de leur construction et de leur utilisation. L'ensemble de critères le plus fréquemment utilisé pour évaluer la qualité des indicateurs choisis est le suivant :

- Clairs : Les indicateurs doivent être précis et clairs. Ils doivent clairement indiquer les aspects qu'ils mesurent, comme les délais, la qualité, la quantité, etc. Ils doivent aussi clairement indiquer s'il existe ou non un biais ou une limite systémique à ce qu'ils peuvent mesurer.

- Pertinents : Les indicateurs doivent être en lien avec, et adaptés à, l'objectif stratégique qu'ils mesurent.

- Économiques : Les informations permettant de développer l'indicateur doivent pouvoir être obtenues à un coût raisonnable en termes de budget et de ressources humaines.

- Exploitables : Les indicateurs et leurs cibles doivent inciter les décideurs à mener des actions pour améliorer la situation, ou, si la situation va dans le mauvais sens, à mener des actions correctives.

- Contrôlables : Les indicateurs doivent être facilement vérifiables et soumis à des validations indépendantes (s'ils sont mesurés de façon indépendante, d'autres usagers doivent obtenir le même résultat à partir des mêmes données brutes).

- Participatifs: Les indicateurs doivent être élaborés et utilisés avec les bénéficiaires finaux (qui sont, en fin de compte, les citoyens) ${ }^{35}$.

- Interprétés et communicables: Les indicateurs doivent être expliqués ou interprétés pour différentes parties prenantes. Pris isolément, les chiffres ne sont pas significatifs : le contexte et les conséquences de la valeur d'un indicateur doivent être expliqués dans les conclusions analytiques des rapports.

- Désagrégés : Il doit être possible de ventiler les indicateurs pour différents groupes selon le sexe, le revenu, etc.

${ }^{35}$ Ce critère n'est néanmoins pas souvent applicable, en particulier dans le cas d'indicateurs de nature technique, par exemple portant sur la dette publique, lorsque les citoyens ne peuvent participer à leur construction. Il est en outre probable que l'élaboration comme l'évaluation des mesures basées sur l'expertise ne puissent s'accompagner d'une large participation citoyenne. 
De la même manière que les critères énumérés ci-dessus, les principes RACER ${ }^{36}$ d'établissement d'indicateurs peuvent être appliqués. Ce schéma préconise de vérifier la pertinence, l'acceptation, la crédibilité, la facilité et la solidité d'un indicateur ${ }^{37}$.

Un ensemble d'indicateurs spécifiques à la RAP est présenté dans l'Annexe 2.

\section{4. Établir des valeurs de référence et des valeurs cibles}

Une fois qu'un ensemble d'indicateurs clés a été défini pour chaque objectif et action, l'étape suivante est de spécifier le niveau de performance. Ceci implique de définir des cibles de performance adaptées aux références actuelles (ou historiques). Les cibles de performance déterminent le niveau fixé de performance que l'administration publique vise à atteindre grâce à la mise en œuvre de la stratégie dans un délai précis.

La première étape pour établir des cibles de performance est d'identifier une valeur de référence, exprimant l'état actuel de la situation (dans l'idéal, la performance actuelle ou historique de l'aspect mesuré). La valeur de référence peut provenir de différentes sources telles que les statistiques nationales, les données administratives et les sources internationales (par exemple, dans le cas de la RAP, les Indicateurs de gouvernance mondiale ou l'Indice de compétitivité mondiale de la Banque mondiale, les rapports d'évaluation par pays SIGMA ou le rapport Panorama des administrations publiques de l'OCDE). Cependant, certains indicateurs pouvant être nouveaux, il sera peut-être nécessaire d'appliquer l'une des options suivantes pour définir la valeur de référence :

- Recueil de nouvelles données et calcul d'une nouvelle valeur. Par exemple, si une stratégie vise, parmi d'autres aspects, à améliorer l'efficacité du travail de l'Agence nationale de protection des données, il faudra chercher à savoir combien de décisions de l'Agence ont été annulées par le Tribunal administratif. Dans ce cas, le calcul de la valeur de référence peut exiger de recueillir des données sur le nombre total de décisions de l'Agence et le nombre de décisions annulées. Ces données sont généralement facilement disponibles et n'exigent pas beaucoup de ressources, mais si les données pour le nouvel indicateur ne sont pas disponibles, ou si elles sont disponibles mais que la méthode de calcul est différente, il pourra être nécessaire de trouver une autre méthode de calcul.

- Établir la valeur de référence à « 0 » lors de l'introduction d'un nouveau système ou processus. Par exemple, si le gouvernement d'un pays prévoit de mettre en place une nouvelle approche pour le recrutement des hauts fonctionnaires par la création d'un nouveau centre d'évaluation, alors le nombre de hauts fonctionnaires recrutés par cette méthode avant la création du centre sera automatiquement fixé à « $0 »$.

36 Pour une lecture complémentaire sur l'approche RACER, voir: Commission européenne (2016), DG NEAR Guidelines on linking planning/programming, monitoring and evaluation, Bruxelles, p. 53.

${ }^{37}$ D'autres critères existent également, comme la fiabilité, la validité ou la faisabilité des données utilisés pour la mesure. Pour des lectures complémentaires sur les autres critères de construction de bons indicateurs, voir Lafortune G., González S., Lonti Z. (2018), Government at a Glance: A Dashboard Approach to Indicators ; Malito D., Umbach G., Bhuta N. (éds) The Palgrave Handbook of Indicators in Global Governance, Palgrave Macmillan, Cham; González, S., L. Fleischer et M. Mira d'Ercole (2017), «Governance statistics in OECD countries and beyond: What exists, and what would be required to assess their quality? ", OECD Statistics Working Papers, No. 2017/03, Éditions OCDE, Paris, https://doi.org/10.1787/c0d45b5e-en. 
- Dans certains cas, il peut être impossible d'établir une valeur de référence, les données étant indisponibles, ou très coûteuses à recueillir rétrospectivement. Dans ces cas, la valeur de référence sera définie comme « non disponible », « non applicable » ou «à déterminer ». Par exemple, si un pays veut réduire sa charge et ses coûts administratifs par l'introduction de la « Méthode des coûts standards » (MCS), il devra calculer le niveau actuel d'économies pour établir la valeur de référence. Or, puisque l'activité l'application de la MCS - est nouvelle, la valeur de référence pourra être définie comme «non disponible ». Dans ce cas, il sera nécessaire de calculer une valeur de référence une fois la MCS mise en place. De la même manière, si le pays envisage de mettre en place de nouvelles activités pour améliorer l'accès aux données par de nouvelles exigences légales sur la fourniture de données sur les portails Internet ministériels, et que, selon le cadre stratégique, cela doive être contrôlé par la mesure des données fournies de manière proactive par les ministères utilisant l'indicateur «Part des ministères et agences fournissant des données numériques de manière proactive, conformément aux exigences légales ", la valeur de référence pourra être définie comme " non applicable », puisqu'aucune institution n'a encore appliqué les nouvelles exigences légales sur la fourniture de données auxquelles l'indicateur fait référence. La valeur de référence ne devrait être définie comme « non disponible » ou « non applicable » que dans des cas exceptionnels. Lorsque ces valeurs sont utilisées, la stratégie doit clairement indiquer quelle année la valeur de référence sera calculée, et tous les efforts doivent être faits pour que cette valeur de référence soit établie le plus tôt possible.

Une fois que la valeur de référence a été établie, l'étape suivante est de fixer la cible. Voici certaines des questions à se poser avant de fixer les valeurs cibles : comment la cible doit-elle être fixée, et jusqu'où peut aller l'ambition? Il n'est pas aisé de décider à quel niveau d'ambition peut se situer la cible, mais pas trop difficile non plus. La cible d'un indicateur cible est habituellement fixée en prenant en compte les informations et les aspects suivants :

- Priorités du gouvernement/de l'institution - l'objectif qui doit être mesuré a-til été déclaré comme une priorité par le gouvernement ou l'institution? Existet-il une pression du public pour significativement améliorer la performance ? Si les objectifs sont considérés comme des priorités ou que la pression publique est forte, alors la cible peut être plus ambitieuse qu'elle ne l'aurait été si l'on s'était contenté d'extrapoler les tendances antérieures. La cible peut alors indiquer une amélioration exponentielle.

- Comparaison entre pairs - quel est le niveau de performance d'autres institutions ou juridictions similaires, et comment la performance du pays doitelle être améliorée s'il veut devenir un chef de file dans les comparaisons entre pairs ? S'il y a de grands écarts par rapport aux pairs, la stratégie peut fixer une cible plus ambitieuse pour réduire ou éliminer ces écarts.

- Ressources disponibles - qu'est-il possible d'accomplir en utilisant les ressources actuelles, et des ressources devraient-elles être réaffectées ? Si la réalisation d'une cible est directement liée à des ressources financières (la numérisation des services publics a, par exemple, des implications financières directes), la cible doit prendre en compte le budget nécessaire prévisible.

- Normes de performance nationales ou internationales - existe-t-il des références établies par des organisations nationales ou internationales pour 
mesurer la performance attendue sur l'aspect considéré du fonctionnement de l'administration publique? Les normes et les cibles sont souvent complémentaires. Par exemple, la norme nationale pour le traitement des demandes de passeports peut être de 2 semaines (hors livraison), et la cible peut être de traiter $95 \%$ des demandes dans ce délai.

- Tendances antérieures - quelle a été l'évolution de la performance au cours des dernières années (trois, cinq années ou plus) et quelles sont les raisons des fluctuations de performance? Quelle sont la taille et la tendance de l'augmentation ou de la diminution annuelle ? Cet aspect a une importance particulière, car améliorer des niveaux de performance existant de longue date nécessite généralement de substantielles ressources supplémentaires et davantage d'attention.

Il faut noter que, pour établir des valeurs cibles réalistes, il est crucial de connaitre la valeur de référence, et aussi, voire surtout, l'évolution de la performance pour les années précédentes. En l'absence de données historiques solides, les cibles peuvent être trop ambitieuses, ou trop faciles à atteindre. Étant donné que les cibles sont surveillées et soumises au contrôle de parties prenantes externes, les organismes ont souvent tendance à fixer des cibles artificiellement basses, pour éviter la critique. De telles cibles devraient être remises en question et corrigées par l'institution chef de file de la réforme, un conseil sectoriel ou conseil de la réforme, ou des structures similaires chargées de garantir la qualité de la stratégie et de piloter tout le processus de réforme. De la même manière, il faut éviter de définir des cibles trop ambitieuses, et les structures en question devraient toujours apprécier le réalisme compte tenu des ressources disponibles et du contexte du pays.

Les cibles peuvent être établies selon plusieurs échéances : annuelle, de moyen terme et dernière année, ou uniquement pour la dernière année. Les cibles intermédiaires (annuelles ou à moyen terme) sont des étapes cibles. Pour responsabiliser l'administration les cibles portant sur la dernière année (Option 3) ne devraient pas être les cibles principales dans un ensemble de cibles. Les cibles définissant la valeur des indicateurs de niveau de résultat (le type d'indicateurs le plus largement utilisé pour vérifier la réalisation des objectifs de la réforme) devraient au moins être établies pour le moyen terme et la dernière année de la stratégie afin de pouvoir évaluer la réalisation des objectifs et le succès global de la mise en œuvre de la stratégie. Lorsqu'elles ont été prévues et fixées, il est possible de mettre en place en parallèle des cibles d'impact ne concernant que la dernière année, en utilisant des techniques d'évaluation, comme il est expliqué dans la Section VIII. L'encadré 10 expose certains des avantages et des limites, associés à chaque option. 


\section{Encadré 10. Avantages et limites des différentes options relatives à l'échéance des cibles}

\section{OPTION 1 : Annuelle}

Les cibles sont établies pour chaque année de validité de la stratégie. Elles sont habituellement fixées pour des indicateurs de réalisation, plus rarement pour des indicateurs de résultat, et ne sont pas conseillées pour mesurer l'impact.

Avantages : fournit un cadre de responsabilisation et de contrôle solide, en renforçant la motivation des institutions chargées de la mise en œuvre.

Limites : très consommatrices en temps de travail, car des changements de contexte peuvent nécessiter de les modifier. De plus, les changements n'étant pas toujours perceptibles d'une année sur l'autre, les efforts pour mesurer la performance à l'aide de cibles annuelles peuvent sembler disproportionnés par rapport à l'information obtenue.

Exemples : Stratégie d'amélioration de la réglementation du Kosovo ${ }^{38}$ (pour les réalisations).

\section{OPTION 2 : Moyen terme et dernière année}

Les cibles sont établies pour une année-étape de moyen terme et pour la dernière année du calendrier de mise en œuvre de la stratégie. Elles sont habituellement appliquées à des indicateurs de résultat, mais aussi parfois pour des mesures d'impact, à condition que la mesure de l'impact partiel soit significative.

Avantages : moins consommatrices en temps de travail que les cibles annuelles, elles facilitent la réactivité par rapport aux changements de contexte plus facile.

Limites: moindres responsabilisation et motivation pour obtenir des résultats. Possibilités limitées de prendre des mesures correctives si les cibles ne sont pas atteintes.

Exemples: Stratégie transversale de RAP 2015-2020 de l'Albanie, Stratégie de RAP de la Moldavie.

\section{OPTION 3 : Dernière année}

Les cibles ne sont définies que pour la dernière année de validité de la stratégie. Elles peuvent être utilisées pour mesurer les impacts mais ne sont pas conseillées pour évaluer les résultats, et ne contribuent pas au contrôle efficace de la production.

Avantages : moins consommatrices en temps de travail, elles facilitent la réactivité par rapport aux changements de contexte.

Limites : faible responsabilisation et motivation pour obtenir des résultats. Pas de possibilité de prendre des mesures correctives pendant la mise en œuvre de la stratégie.

Exemples : Programme national des réformes majeures de la Tunisie.

Les indicateurs stratégiques, ainsi que leurs valeurs cibles et de référence, peuvent être présentés de diverses façons dans un document exposant une stratégie. L'encadré 11 présente certaines de ces options dans le cas d'une stratégie liée à la RAP. 
Encadré 11. Options de présentation des indicateurs et de leurs valeurs cibles et de référence dans les documents de stratégie de RAP OPTION 1 : Dans le texte sur la stratégie de RAP, avec les objectifs

Les indicateurs, avec leurs valeurs cibles et de référence, peuvent être présentés dans le texte sur la stratégie de RAP, juste après les objectifs définis. Il est souhaitable que cette information soit présentée sous la forme d'un tableau, pour montrer clairement les liens entre objectifs et indicateurs, et faciliter la présentation de leurs valeurs de référence et cibles.

Avantages : l'information sur les objectifs et les niveaux attendus de performance est présentée dans un seul document et permet d'établir des liens directs entre les deux.

Limites : le document sur la stratégie de RAP peut sembler plus long et plus technique. Cette option est également moins flexible, et les révisions de cibles peuvent être moins fréquentes que lorsqu'elles sont présentées seulement comme une partie d'un plan d'action.

Exemples : Stratégie de RAP du Monténégro 2016 - 2020.

OPTION 2 : Dans le plan d'action (ou feuille de route) de la stratégie de RAP

Les indicateurs, avec leurs valeurs cibles et de référence, peuvent être présentés dans le plan d'action de la stratégie de RAP.

Avantages : assez flexible pour permettre la révision des cibles en cas de changement de contexte. Le document concernant la stratégie lui-même est plus concis.

Limites : l'information sur les objectifs est répartie sur deux documents. Il n'y a pas de lien direct, visuel entre les objectifs retenus et leur cadre de mesure.

Exemples : Stratégie de RAP de la Serbie et son plan d'action 2015 $2017^{39}$.

\section{OPTION 3 : Dans un document distinct, annexé à la stratégie de RAP}

Les indicateurs, avec leurs valeurs cibles et de référence, peuvent être présentés dans un tableau distinct qui peut être annexé à la stratégie de RAP. Dans ce cas, l'annexe devrait avoir le statut de partie officielle de la stratégie de RAP.

Avantages : suffisamment flexible pour permettre la révision des cibles lors des changements du contexte, et constitue une stratégie de RAP et un plan d'action plus faciles à utiliser.

Limites : l'information sur les objectifs est répartie sur plusieurs documents. Il n'y a pas de lien direct, visuel entre les objectifs retenus et leur cadre de mesure.

38 Better Regulation Strategy 2.0 for Kosovo 2017-2021, décembre 2016, http://www.kryeministri-ks.net/repository/docs/Better_Regulation_Strategy_2_0_for_Kosovo_ENGLISH.pdf.

39 Action Plan for the Implementation of the Public Administration Reform Strategy in the Republic of Serbia, 2015-2017, http://www.mduls.gov.rs/english/reforma-javne-uprave.php. 
Exemples : Stratégie transversale de RAP de l'Albanie 2015 - 2020.

L'Annexe 2 de ce Manuel présente un modèle, élaboré par SIGMA, de présentation des indicateurs dans une stratégie ou un plan d'action de RAP. Il est également applicable à toute autre stratégie sectorielle. Les indicateurs peuvent, bien sûr, être présentés sous n'importe quel format convenant au pays concerné, pour autant que soit fournie l'information essentielle concernant les indicateurs, les valeurs de référence et les valeurs cibles - quelle que soit la méthode de présentation retenue.

\subsection{Utilisation de cartes d'identité des indicateurs}

L'expérience montre que, pour utiliser un indicateur, il ne suffit pas de s'accorder sur sa définition, sa valeur de référence et ses cibles. Cela exige des précisions et un consensus sur la manière dont les valeurs seront calculées, sur les données qui devront être recueillies, par qui et quand, et sur les sources qui seront utilisées. L'utilisation de cartes d'identité des indicateurs constitue un cadre pratique pour systématiser les données et leur recueil, et augmenter la disponibilité et la qualité des données.

Le défi principal le plus souvent rencontré lors de l'utilisation d'indicateurs dans la mise en œuvre et le suivi d'une stratégie réside dans le fait que les institutions chargées de fournir les données, ou de mesurer un indicateur donné, ne savent pas comment calculer les valeurs de l'indicateur, ni quelles seront les données nécessaires dans ce but, ni précisément ce que l'indicateur mesure.

En conséquence, les organismes responsables de l'indicateur peuvent déclarer qu'il n'est pas possible de fournir des informations sur lui, qu'il n'est pas pertinent, qu'il n'est pas possible de recueillir les données, ou que l'indicateur n'est pas tel qu'il était prévu lors de l'élaboration de la stratégie et du plan d'action. L'élaboration de la carte d'identité d'un indicateur est un moyen de réduire le risque qu'un tel scénario se produise.

La carte d'identité de l'indicateur fait partie intégrante de la documentation sur la stratégie, elle fournit des détails sur chaque indicateur afin de rendre le cadre de suivi de la performance plus solide et fiable. Son but n'est pas seulement de clarifier la compréhension, l'interprétation, la portée, et le processus exact de calcul de chaque indicateur en interne (c'est-à-dire parmi les personnes responsables de la mise en œuvre et les institutions chargées de fournir et mesurer les données), mais aussi d'aider les lecteurs externes à savoir exactement ce que l'indicateur retenu mesure, et ce qui peut être attendu ou non du cadre de suivi de la performance. C'est la raison pour laquelle cette carte d'identité des indicateurs stratégiques devrait être rendue accessible au public (c'est-à-dire publiée), ce qui d'ailleurs renforcerait la crédibilité et la transparence du processus de réforme dans son intégralité.

La carte d'identité peut couvrir les aspects suivants :

- titre de l'indicateur

- lien avec les objectifs

- courte définition de l'indicateur

- source, méthode de recueil et fréquence de recueil des données

- institution(s) chargée(s) de recueillir les données nécessaires

- méthode de calcul des valeurs de l'indicateur (sous la forme d'une formule si nécessaire) 
- valeur de référence de l'indicateur, valeurs cibles intermédiaires et finale

- difficulté anticipée du recueil de données et solutions possibles (ainsi que tout biais ou limite systémique)

- information sur l'évolution de la performance au cours des quelques années précédentes ${ }^{40}$

La carte d'identité peut et doit être utilisée lors de différentes étapes de l'élaboration et de la mise en œuvre de la stratégie :

- Planification : communique et explique, aidant les lecteurs à comprendre l'information sur la performance

- Suivi et reporting: clarifie l'information sur la performance et facilite l'évaluation de la performance par rapport aux cibles

- Évaluation : aide à la compréhension et à la clarification de l'information sur la performance, et facilite l'évaluation de la performance et de l'impact général des réformes

- Vérification : aide à la compréhension et à la clarification de l'information sur la performance, et à la vérification des résultats allégués des réformes

On donne plusieurs noms à ce type de description détaillée des indicateurs retenus, les plus fréquents étant « carte d'identité de l'indicateur », « note technique », « méthode de mesure » et « profil d'indicateur ».

L'Annexe 2 de ce Manuel présente un modèle élaboré par SIGMA ainsi qu'une carte d'identité d'indicateur.

\section{Réflexions pratiques :}

$\checkmark$ Évitez de surdévelopper la liste d'indicateurs - si un indicateur suffit à mesurer le succès de l'objectif ou de l'action (en saisissant son essence), il n'est pas besoin d'en construire un autre à tout prix. Tâchez d'être économe, car la mesure et le suivi régulier demandent du temps et des efforts.

$\checkmark$ Des indicateurs déjà prêts, saisissant parfaitement la nature de l'objectif ou de l'action concernés, sont très souvent disponibles dans les statistiques existantes, les sources internationales ou les autres programmes similaires. Si ce n'est pas le cas, il sera nécessaire de construire de nouveaux indicateurs sur mesure. Ne soyez pas tenté de gagner du temps en créant des liens artificiels avec des indicateurs existants, mais en fait non pertinents. Cela ne ferait que créer une charge inutile.

$\checkmark$ Il faudra souvent attribuer des budgets supplémentaires pour couvrir les coûts de l'élaboration d'une nouvelle méthodologie ou ceux du recueil de données (par exemple, pour mesurer objectivement la satisfaction des usagers).

$\checkmark$ La carte d'identité d'un indicateur est d'une utilité étonnante! Elle peut permettre de tester si les indicateurs de performance peuvent être utilisés en pratique, et de voir s'ils signifient vraiment quelque chose pour ceux qui doivent les utiliser. L'établir permet in fine d'obtenir un consensus sur ce qui doit être mesuré, par qui, quand et comment, et aide aussi à voir quand le calcul de certains indicateurs n'est

${ }^{40}$ Les aspects énumérés ci-dessus sont également souvent connus comme les métadonnées de l'indicateur. 
pas réaliste, ou s'il faut modifier des procédures internes pour obtenir les données nécessaires.

\section{Ne pas oublier :}

Les indicateurs doivent être directement en lien avec les objectifs et les actions.

$\checkmark$ Le nombre d'indicateurs permettant de saisir l'essence d'un objectif donné devrait être rationnellement limité. À moins que le résultat espéré soit obtenu par un ensemble d'actions - auquel cas un indicateur peut suffire, à chaque action devrait correspondre (au moins) un indicateur, qui saisisse ce qu'elle a permis de réaliser.

$\checkmark$ Tous les objectifs ou actions devraient, d'une façon ou d'une autre, être mesurés.

$\checkmark$ Dans l'idéal, chaque indicateur devrait avoir une valeur de référence et une (ou des) valeur(s) cible(s).

$\checkmark$ Les personnes chargées du recueil de données, du suivi régulier, du reporting (de la rédaction des rapports et de l'analyse devraient être clairement mentionnées dans la stratégie ou dans les documents qui s'y réfèrent.

$\checkmark$ Chaque indicateur, si la décision en est prise, devrait avoir une carte d'identité détaillant les éléments principaux le concernant: mesure, utilisation, méthode de calcul, etc.

$\checkmark$ Les détails concernant les indicateurs retenus devraient être rendus publics pour renforcer la transparence et la crédibilité.

$\checkmark$ Les indicateurs et leurs cartes d'identité doivent être construits en même temps que la stratégie, et non après son adoption. 


\section{5. Élaboration du plan d'action}

Une fois que les objectifs stratégiques ont été définis et que les indicateurs et leurs cibles ont été fixés, l'étape suivante est de planifier les actions spécifiques qui doivent être mises en œuvre ou menées afin d'atteindre les objectifs et les cibles fixés : autrement dit, d'élaborer un plan d'action.

Les actions peuvent être définies comme les moyens par lesquels un pays (généralement, mais pas exclusivement, le gouvernement de ce pays) met en œuvre ses politiques. Une action est ce qui permet de mettre en œuvre un projet ou de réussir une réforme. Il s'agit d'une activité ou d'un ensemble d'activités et de mesures concrètes, directement liées à l'utilisation des intrants (ressources humaines, financières et matérielles), afin de livrer certains produits prédéfinis ou d'atteindre des objectifs politiques spécifiques. Une action ne peut être considérée comme concrète que si la responsabilité de sa mise en œuvre est clairement définie. Cette responsabilité peut incomber au niveau institutionnel comme au niveau managérial (idéalement, l'institution, le responsable ou la structure responsable seront précisés). Chaque action doit aussi bénéficier de ressources pour être menée à bien, et le calcul du coût des ressources nécessaires est nécessaire lors de sa préparation (la Section VI revient en détail sur ce sujet). Des exemples théoriques d'activités concrètes liées à la RAP et de leurs principaux éléments sont présentés dans le Tableau 3.

Tableau 3. Exemples théoriques d'activités concrètes liées à la RAP

\begin{tabular}{|l|l|l|l|l|}
\hline Activité & Produit & Responsable & Délai & Ressources en \\
EUR
\end{tabular}

Il est important de limiter le nombre d'actions afin de concentrer les efforts (et les ressources financières) sur la mise en œuvre de celles qui auront le plus d'impact. Une stratégie est un document de haut niveau, à moyen ou long terme, qui n'a pas besoin de présenter tous les détails de chaque activité permettant d'atteindre les objectifs. S'il y a 
trop de détails, les rédacteurs et les futurs responsables de la mise en œuvre risquent de perdre le fil, et en fin de compte la réforme pourrait ne pas s'attacher à la réalisation des actions les plus importantes, exhaustives ou efficaces, qui ont le plus de potentiel pour susciter un changement.

Les actions devraient, autant que possible, être centrées sur la réforme, nouvelles et innovantes. Elles devraient aussi permettre de résoudre efficacement les problèmes identifiés pendant la phase d'analyse de problèmes, ainsi que leurs causes, l'ensemble ayant été examiné dans l'analyse par arbre à problèmes, si cette méthode est utilisée. Il convient d'éviter de retenir des actions qui sont déjà mises en œuvre, ou devraient l'être. Autrement dit, les actions devraient être de nouveaux programmes, activités, projets et mesures axés sur la réforme, qui apportent un changement significatif ou améliorent des pratiques ou systèmes existant. On peut citer comme exemples d'actions axées sur la réforme sont: "Concevoir et créer un Système informatique pour la gestion des ressources humaines et le rendre pleinement opérationnel», «Établir et adopter un nouveau système de rémunération », " Développer une méthodologie pour concevoir des documents de planification stratégique, suivre leur mise en œuvre et procéder à des évaluations d'impact», «Établir quatre centres de services pilotes comme guichets uniques », etc.

L'Encadré 12 présente la liste des questions de contrôle permettant de vérifier si une action est nouvelle et innovante ou non, et si elle a le potentiel d'engendrer le niveau attendu d'impact ou de changement. Il s'agit de questions initiales, et cette liste ne doit en aucun cas être considérée comme exhaustive.

\section{Encadré 12. Liste de questions pour tester la nouveauté et l'impact potentiel des actions}

1. L'action est-elle nouvelle ou a-t-elle un quelconque ?

2. L'action change-t-elle tout le système, ou l'un ou plus de ses éléments?

3. L'action est-elle simple ou complexe en termes de mise en œuvre et de variété de diversité de facteurs sur lesquels elle aura un impact?

4. L'action est-elle complète (c'est-à-dire un ensemble d'activités liées entre elles)?

5. Quelle est la taille du groupe cible qui sera affecté par le changement ou la nouvelle action?

6. Le changement est-il significatif? Affectera-t-il uniquement les facteurs internes comme les procédures administratives, ou aura-t-il également un impact sur l'environnement externe en termes de facteurs tels que la qualité et la rapidité des services?

7. Quelle est l'importance du budget consacré à la mise en œuvre de l'action?

8. Combien faut-il de temps pour mettre en œuvre l'action?

\subsection{Types d'actions}

Pour identifier les actions, il faut réfléchir aux différents moyens politiques qui pourraient être appliqués par le secteur public pour résoudre les problèmes. Les moyens politiques les plus communément utilisés par les gouvernements sont présentés dans l'Encadré 13. 


\begin{tabular}{|c|c|}
\hline \multicolumn{2}{|c|}{ Encadré 13. Exemples de moyens politiques liés à la RAP } \\
\hline Moyen politique & Exemples (liés à la RAP) : \\
\hline $\begin{array}{l}\text { Moyens réglementaires - } \\
\text { définissent des droits, obligations, } \\
\text { restrictions ou normes stipulés } \\
\text { dans les lois et réglementations }\end{array}$ & $\begin{array}{l}\text { - Révision du cadre législatif, pour } \\
\text { inclure les administrations fiscale et } \\
\text { douanière ainsi que le corps } \\
\text { diplomatique, dans le domaine de la } \\
\text { fonction publique } \\
\text { - Révision du cadre juridique pour } \\
\text { limiter les marchés publics non } \\
\text { concurrentiels } \\
\text { Introduction dans les règles de } \\
\text { procédure administrative de } \\
\text { l'obligation de mener des études } \\
\text { d'impact de la réglementation lors de } \\
\text { l'élaboration d'une nouvelle } \\
\text { législation }\end{array}$ \\
\hline $\begin{array}{l}\text { Moyens administratifs - y } \\
\text { compris la fourniture directe ou } \\
\text { indirecte (externalisée) de services, } \\
\text { l'investissement en infrastructures, } \\
\text { le développement des compétences } \\
\text { (formation), l'inspection, etc. }\end{array}$ & $\begin{array}{l}\text { - Introduction de solutions } \\
\text { technologiques d'interopérabilité pour } \\
\text { permettre l'échange illimité de } \\
\text { documents entre différents registres et } \\
\text { institutions } \\
\text { - Création d'un centre d'évaluation } \\
\text { pour le recrutement centralisé des } \\
\text { hauts responsables } \\
\text { - Formation des hauts fonctionnaires en } \\
\text { gestion optimisée des ressources } \\
\text { humaines } \\
\text { Renforcement des contrôles fiscaux } \\
\text { dans des secteurs ciblés pour réduire } \\
\text { l'économie informelle }\end{array}$ \\
\hline $\begin{array}{l}\text { Moyens informatifs - incluent les } \\
\text { campagnes de diffusion } \\
\text { d'informations, les publications, le } \\
\text { développement de nouveaux sites } \\
\text { internet d'information, etc. }\end{array}$ & $\begin{array}{l}\text { - Conception et exécution d'une } \\
\text { campagne nationale pour faire } \\
\text { augmenter l'utilisation des services } \\
\text { numériques parmi les citoyens } \\
\text { - Conception et exécution de sessions } \\
\text { d'information sur l'importance de la } \\
\text { protection des données pour les }\end{array}$ \\
\hline
\end{tabular}




\begin{tabular}{|c|c|}
\hline & $\begin{array}{l}\text { principales organisations } \\
\text { gouvernementales } \\
\text { - Ligne téléphonique pour informer les } \\
\text { citoyens sur les mesures de lutte } \\
\text { contre la corruption }\end{array}$ \\
\hline $\begin{array}{l}\text { Moyens institutionnels - comprennent la } \\
\text { création ou la réorganisation d'organismes } \\
\text { publics pour que soit réellement assurées } \\
\text { les tâches fondamentales de l'État ou la } \\
\text { fourniture de services publics, et pour } \\
\text { améliorer la qualité des services }\end{array}$ & $\begin{array}{l}\text { - Fusion des structures chargées des } \\
\text { marchés publics en une structure } \\
\text { centralisée } \\
\text { - Examen fonctionnel complet des } \\
\text { fonctions de TI des ministères et } \\
\text { agences du gouvernement et } \\
\text { recommandations pour leur } \\
\text { rationalisation }\end{array}$ \\
\hline $\begin{array}{l}\text { Moyens financiers - incluent les } \\
\text { subventions, les déductions fiscales, les } \\
\text { assurances, les conditions commerciales } \\
\text { favorables, etc. Ils sont cependant } \\
\text { rarement utilisés dans la RAP, bien que de } \\
\text { nouvelles amendes ou incitations } \\
\text { financières puissent être introduites }\end{array}$ & $\begin{array}{l}\text { - Augmentation des amendes pour } \\
\text { violation des exigences de protection } \\
\text { des données de la part des détenteurs } \\
\text { de données publics et privés }\end{array}$ \\
\hline
\end{tabular}

Ces moyens politiques servent de cadre à l'aune duquel examiner les mesures possibles et optimales pour résoudre les problèmes identifiés et sélectionnés. Très souvent, il est possible d'accomplir les objectifs grâce à une combinaison de divers moyens politiques. Les actions peuvent également être liées entre elles : pour que l'une ait lieu, il faut qu'une autre ait au préalable été mise en œuvre.

\subsection{Le processus de formulation des actions}

Il est très difficile d'identifier et de conceptualiser les actions qui sont axées sur la réforme et ont un impact significatif. Souvent, l'action proposée n'a qu'un impact limité et administratif. Les institutions concernées devraient donc consacrer un temps suffisant et organiser plusieurs sessions interactives (telles que des réunions de groupes de travail, de groupes de réflexion et des sessions de brainstorming) pour garantir que les résultats de l'analyse de problèmes, les problèmes identifiés et leurs causes sont compris, et qu'a été arrêté le choix le meilleur ensemble d'actions favorisant la réforme. La programmation des actions doit aussi comprendre une synthèse stratégique justifiant la hiérarchie et la chronologie des actions - pourquoi une action spécifique est cohérente, ce qu'elle implique en termes de compromis et de risques, et les conséquences de la démarche retenue. Ces motifs doivent être exposés dans la stratégie lorsqu'est écrite l'orientation principale de l'action proposée.

L'expérience de la RAP de SIGMA montre que les représentants des institutions clés sont souvent mal à l'aise lorsqu'ils ne doivent proposer qu'un petit nombre d'actions, et qu'ils suggèrent donc souvent une longue liste d'activités, y compris des activités qui ne sont pas nouvelles et ne correspondent pas aux principes d'un plan d'action de stratégie de RAP. De la même façon, il est assez fréquent que les actions proposées soient trop 
diffuses, et que beaucoup d'entre elles ne soient pas directement liées à un (des) problème(s) défini(s). Par conséquent, les rédacteurs des actions doivent toujours vérifier, au cours de nombreuses discussions, que les actions proposées traitent le(s) problème(s) identifié(s), sont vraiment essentielles et sont applicables de manière réaliste.

Afin de relever ces défis, chaque activité proposée doit être évaluée pour savoir si elle serait menée indépendamment de sa présence dans le plan d'action. Si c'est bien le cas, il n'y a pas besoin de l'inclure dans le plan d'action car il s'agit d'une activité habituelle. Par exemple, «Établir un nouveau format pour l'établissement de rapports » est une activité mineure qui est inévitable si un nouveau système de suivi est mis en place. Il faudrait donc plutôt utiliser la formulation suivante: «Mettre en place un nouveau système de suivi fondé sur les informations de performance et les rapports publics ». Afin de prévoir des délais réalistes, il convient d'identifier l'ensemble des actions nécessaires à l'obtention du résultat final, en commençant par le produit final, et d'évaluer ensuite le temps requis pour chaque action.

La préoccupation principale, lors du processus de planification des actions, doit être de concevoir intelligemment une méthode pour atteindre les objectifs en utilisant un nombre limité d'actions axées sur la réforme, qui ciblent les causes profondes du problème. L'Encadré 14 présente des exemples de la manière dont un objectif est traduit en actions de réforme. 


\begin{tabular}{|c|c|}
\hline \multicolumn{2}{|c|}{$\begin{array}{l}\text { Encadré 14. Exemples d'objectifs liés à la RAP et d'actions } \\
\text { indispensables pour les atteindre }\end{array}$} \\
\hline Objectif spécifique & Actions indispensables \\
\hline $\begin{array}{l}\text { Améliorer l'élaboration de politiques } \\
\text { fondée sur des éléments concrets dans } \\
\text { la planification des investissements } \\
\text { publics }\end{array}$ & $\begin{array}{l}\text { 1. Développer et appliquer une nouvelle } \\
\text { méthodologie d'évaluation des projets } \\
\text { d'investissement public (Analyse coûts- } \\
\text { bénéfices), comprenant la formation } \\
\text { approfondie de tout le personnel du } \\
\text { ministère à partir de } 2018 \text {. } \\
\text { 2. Développer et appliquer une Procédure } \\
\text { d'exploitation standardisée pour préparer un } \\
\text { portefeuille de projets d'investissement } \\
\text { public dans tous les secteurs à partir de } \\
\text { 2019, le premier projet devant être } \\
\text { développé avant la fin } 2019 \text {. }\end{array}$ \\
\hline $\begin{array}{l}\text { Accroître l'accès à l'information et aux } \\
\text { données publiques }\end{array}$ & $\begin{array}{l}\text { 1. Obliger toutes les institutions publiques, en } \\
\text { changeant la Loi sur l'accès à l'information, } \\
\text { à fournir l'information de manière proactive } \\
\text { sur leurs sites internet. } \\
\text { 2. } \begin{array}{l}\text { Définir des critères pour la fourniture de } \\
\text { données ouvertes par toutes les institutions } \\
\text { publiques, et obliger toutes les institutions } \\
\text { publiques à commencer à fournir des } \\
\text { données ouvertes avant la fin } 2019 .\end{array} \\
\text { 3. } \begin{array}{l}\text { Concevoir et lancer un portail centralisé de } \\
\text { données ouvertes avant la fin } 2020 .\end{array}\end{array}$ \\
\hline
\end{tabular}

L'un des défis de la planification d'actions est de définir des actions claires, concises et bien écrites, qui soient compréhensibles pour tous les lecteurs externes. La formulation d'une action est souvent longue, maladroite et technique. L'action ne doit pas être formulée comme un acte juridique ou un travail scientifique. L'Encadré 15 présente des exemples d'actions bien et mal formulées.

Encadré 15. Exemples d'actions mal et bien formulées (exemples de RAP)

\begin{tabular}{|l|l|}
\hline \multicolumn{1}{|c|}{ Actions mal formulées } & \multicolumn{1}{|c|}{ Actions bien formulées (réécrites) } \\
\hline $\begin{array}{l}\text { Création d'une étude sur la performance } \\
\text { de la gestion dans l'administration } \\
\text { publique comme base de départ à la }\end{array}$ & $\begin{array}{l}\text { Rationnaliser les systèmes de gestion de la } \\
\text { performance (planification de politiques, } \\
\text { gestion des ressources humaines, }\end{array}$ \\
\hline
\end{tabular}




\begin{tabular}{|c|c|}
\hline $\begin{array}{l}\text { réunion des différentes méthodes de } \\
\text { gestion de la performance } 41 \text { en un seul } \\
\text { système cohérent, avec des } \\
\text { recommandations d'amendements au } \\
\text { cadre juridique. }\end{array}$ & $\begin{array}{l}\text { budgétisation des programmes, contrôle } \\
\text { interne) sur la base des bonnes pratiques } \\
\text { internationales et en réalisant les } \\
\text { modifications juridiques nécessaires. }\end{array}$ \\
\hline $\begin{array}{l}\text { Activités d'administration en ligne - mise } \\
\text { en œuvre du système de gestion } \\
\text { électronique des documents (GED) dans } \\
\text { tous les ministères et au sein du Secrétariat } \\
\text { général (Étape 1). }\end{array}$ & $\begin{array}{l}\text { Étendre l'application du système de GED à } \\
\text { tous les ministères et au Secrétariat général } \\
\text { (Étape } 1) \text {. }\end{array}$ \\
\hline $\begin{array}{l}\text { Accroître la démocratie numérique en } \\
\text { augmentant la participation électronique. }\end{array}$ & $\begin{array}{l}\text { Accroître l'usage des consultations publiques } \\
\text { par des plateformes de participation en ligne } \\
\text { pour améliorer l'information. }\end{array}$ \\
\hline $\begin{array}{l}\text { Élaboration et adoption de documents } \\
\text { stratégiques (documents conceptuels) pour } \\
\text { la mise en place d'un système de service } \\
\text { public instituant des critères concernant le } \\
\text { recrutement et les promotions en accord } \\
\text { avec les principes de transparence, de } \\
\text { concurrence et de mérite, et avec des } \\
\text { recommandations pour l'amélioration de } \\
\text { tous les domaines du système } \\
\text { d'administration publique. }\end{array}$ & $\begin{array}{l}\text { Élaborer les actes juridiques nécessaires pour } \\
\text { mettre en place un système de recrutement et } \\
\text { de promotion transparent, concurrentiel et } \\
\text { fondé sur le mérite dans la fonction publique, } \\
\text { portant sur tous les domaines de } \\
\text { l'administration publique et fondé sur les } \\
\text { bonnes pratiques. }\end{array}$ \\
\hline
\end{tabular}

\subsection{Le document du plan d'action}

Les actions sont détaillées dans un plan d'action, qui ne devrait en mentionner que les orientations principales. Ce plan peut toutefois être complété par des documents internes additionnels énumérant les étapes à suivre pour mettre en œuvre chaque action. De tels documents peuvent être utiles lors du chiffrage du plan d'action, étant donné que, dans certains cas, celui-ci ne fournit pas un niveau de détails suffisant pour identifier tous les intrants nécessaires. Ils aideront également les institutions à savoir si les actions envisagées sont réalistes au vu des ressources financières disponibles et des délais prévus pour leur exécution. Néanmoins, le but ne doit pas être d'inclure ces documents dans la stratégie, cela ne ferait que surcharger les décideurs, ainsi que la plupart des lecteurs externes.

L'élaboration d'un plan d'action devrait impliquer, outre les représentants des institutions gouvernementales, des parties prenantes externes (comme des ONG, des

${ }^{41}$ Responsabilité organisationnelle ; gestion des politiques publiques - planification stratégique ; gestion des finances publiques et du programme budgétaire; gestion des risques et contrôle interne ; gestion des ressources humaines et évaluation des fonctionnaires. 
associations, des leaders d'opinion dans certains domaines, des experts et des universitaires) qui peuvent valider l'orientation proposée, voire suggérer des solutions additionnelles, plus innovantes et centrées sur l'usager que celles qui étaient initialement avancées. L'élaboration d'un plan d'action est un processus itératif qui exige plusieurs sessions de discussion et d'ajustements.

Par exemple, cette approche est bien illustrée par l'élaboration du plan d'action pour la Stratégie de RAP de la Serbie en 2017 : le ministère principal chargé de la réforme a publié un appel public aux organisations non étatiques pour qu'elles se joignent aux divers groupes thématiques élaborant les différentes parties du nouveau plan d'action. Les organisations de la société civile étaient des membres à part entière des groupes thématiques, et leurs contributions ne servaient pas qu'à contrôler le processus, mais à participer activement à l'élaboration des activités, objectifs et indicateurs.

Le plan d'action est habituellement préparé sous la forme d'un tableau reflétant les axes politiques principaux de la stratégie de RAP (c'est-à-dire les différents niveaux d'objectifs et leurs indicateurs respectifs) et incluant toutes les informations nécessaires à l'efficience de leur mise en œuvre, de leur suivi et de leur contrôle, ces éléments étant clairement liés aux objectifs qu'ils visent à étayer. Ce tableau inclut :

- une brève description de l'action

- le(s) délai(s) de mise en œuvre (qui peuvent être multiples ou échelonnés et, si un suivi rigoureux et des rapports sont exigés, détaillés par trimestre ou par mois)

- les ressources financières et humaines requises pour la mise en œuvre, et les sources de financement

- les entités/les structures chargées de la mise en œuvre, et toute autre institution contribuant à celle-ci

- des indicateurs de réalisation ou de processus pour chaque action

Le délai d'exécution du plan d'action peut être établi de plusieurs manières, selon la validité des objectifs. Cependant, la planification d'actions est plus réaliste quand le délai est fixé entre un et trois ans. Ce qui signifie également que, si le délai de la stratégie est plus long, des plans d'action ultérieurs peuvent être élaborés. Lorsque la fin du plan d'action initial approche, l'élaboration d'un nouveau plan permet d'examiner la pertinence des objectifs initiaux, toute modification importante des facteurs externes pesant sur la réforme, toute évolution des problèmes initialement identifiés, et toute nouvelle priorité de réforme. Il faut aussi mener une évaluation minutieuse du caractère réaliste des ambitions de mise en œuvre du plan d'action original afin que les actions puissent être remaniées si nécessaire. Les pays définissent différentes durées pour les plans d'action, elle peut être par exemple de deux ans (cas du Monténégro et de la Géorgie), sur trois ans (par ex. Albanie, Serbie), mais aussi, mais plus rarement, sur cinq ans (par ex. Ukraine). Dans certains cas rares, les pays n'indiquent pas de délai exact pour la mise en œuvre de la stratégie, même s'ils élaborent des plans d'action consécutifs (par ex. la Stratégie de RAP de la Serbie).

Le plan d'action devrait être préparé, et idéalement adopté aussi, en même temps que la stratégie, ou immédiatement après l'adoption de la stratégie (ce cas doit rester exceptionnel, doit être expressément motivé et s'accompagner d'un engagement ferme sur une date d'adoption future). Les actions retenues par le plan d'action ont un impact direct sur la réalisation des objectifs envisagés, elles devraient donc aussi influencer l'établissement des cibles d'indicateurs correspondantes. De plus, le budget pour la mise en œuvre de la stratégie ne peut être calculé qu'une fois le plan d'action rédigé. Les 
ressources financières disponibles pour l'exécution de la stratégie ont également un impact direct sur ce qui peut être planifié de manière réaliste. C'est pourquoi l'adoption parallèle du plan d'action de la stratégie augmente la faisabilité financière du processus global de réforme de l'administration publique et permet de fixer des objectifs et des cibles plus réalistes. Néanmoins, cela requiert une discipline, une planification et un engagement forts de la part des institutions principales et participantes sont indispensables pour parvenir à élaborer en même temps la stratégie et le plan d'action.

À l'inverse, il faut éviter de préparer et adopter le plan d'action après que la stratégie a été adoptée - cela ne devrait arriver que dans certains cas exceptionnels. En effet, cette éventualité pourrait avoir pour conséquences :

- des questions sur la manière dont des engagements généraux seront réellement concrétisés

- une tendance à réviser les cibles si les coûts apparaissent ultérieurement trop élevés

- un impact sur le choix des indicateurs stratégiques, car ils pourrait être nécessaire d'en corriger ou ajuster certains lorsqu'ils sont traduits en activités réalisables

- une révision forcée des priorités due à la découverte ultérieure de l'absence des ressources financières requises pour la mise en œuvre envisagée

Après l'adoption de la stratégie et de son plan d'action, il est de la responsabilité de la (des) principale(s) institution(s) de coordination, et in fine des structures de gestion de la réforme, de s'assurer que les activités envisagées sont dûment reflétées dans les futurs plans d'action annuels et à moyen terme du gouvernement. Il est particulièrement important que la mise en œuvre des réformes envisagées (ainsi que la programmation des ressources nécessaires à leur mise en œuvre) soit constamment validée et soutenue dans ces plans d'action concernant l'ensemble de l'administration.

Afin de faciliter l'élaboration de plan d'actions, SIGMA a développé un modèle de plan d'action, présenté dans l'Annexe $\mathbf{3}$ de ce Manuel avec un exemple de document de plan d'action, afin d'aider au processus de planification des actions.

\section{Réflexions pratiques :}

$\checkmark$ Ne vous inquiétez pas si votre plan d'action est court. La qualité vaut mieux que la quantité. Visez un petit nombre d'actions ayant un impact réel plutôt qu'une longue liste d'actions qui n'auront qu'un effet limité.

$\checkmark$ Soyez créatif et ouvert, et essayez d'organiser des réunions qui permettront de trouver les solutions les meilleures, les plus efficaces et les plus efficientes aux problèmes identifiés. Invitez des parties prenantes externes, car elles ont souvent une approche différente, centrée sur l'usager, et peuvent en conséquence souligner des aspects cruciaux différents et fournir de bonnes solutions.

$\checkmark$ Demandez à quelqu'un qui n'a pas participé à la rédaction de lire le projet de plan d'action. Si la personne comprend ce qui doit être fait pour réaliser les objectifs retenus, alors les actions sont clairement formulées. Sinon, retravaillez le projet pour capturer l'essence des actions envisagées. 
Ne pas oublier :

$\checkmark$ Idéalement, le document du plan d'action doit être préparé parallèlement (ou même avant) à la finalisation et à l'adoption de la stratégie (ou même avant).

$\checkmark$ Les actions doivent être nouvelles, et doivent significativement changer les pratiques ou systèmes en vigueur.

$\checkmark$ Chaque action doit être directement liée à un objectif donné, et doit être conçue de manière à avoir un impact véritable sur les problèmes identifiés et leurs causes profondes. Aucune action ne doit être incluse sans être justifiée, notamment par le problème qu'elle cherche à résoudre et les objectifs qu'elle sert à atteindre.

$\checkmark$ Un plan d'action doit comprendre: 1) la définition de l'action; 2) le délai ou la durée; 3) l'institution ou la structure responsable; 4) le coût et les sources de financement; et 5) les indicateurs de niveau de production ou de processus. 


\section{Chiffrage}

Une stratégie n'est utile que s'il est possible de la mettre en œuvre. L'expérience montre que l'un des principaux obstacles à la réforme est le manque de financement pour réaliser les actions envisagées. L'élaboration d'une stratégie n'est pas seulement un moyen, pour un pays, d'identifier les principaux problèmes et la manière dont il souhaite les résoudre - et dans quelle mesure - mais aussi de prévoir quelles ressources sont requises pour effectuer les changements désirés.

Une fois que les objectifs fixés et les actions indispensables pour les atteindre déterminées, il est crucial d'évaluer les ressources nécessaires à leur mise en œuvre. En outre, ces calculs doivent être comparés au financement disponible évalué avant la finalisation de la stratégie, car les coûts estimés de mise en œuvre des actions envisagées pourraient se révéler supérieurs non seulement aux ressources disponibles, mais aussi aux apports qu'il est possible d'espérer de bailleurs de fonds externes (tels que des partenaires du développement comme l'UE, des pays membres de l'UE, ou des organisations internationales comme le Fonds monétaire international [FMI] ou la Banque mondiale). Dans ce cas, le niveau d'ambition doit être réajusté, peut-être en retardant la mise en œuvre de certaines actions, ou même en modifiant l'ordre de priorité des objectifs que la réforme cherche à atteindre.

Lorsqu'une stratégie est développée, elle doit toujours inclure le calcul correct du coût des ressources requises pour sa mise en œuvre. De plus, des mesures doivent être prises pour s'assurer que les ressources ainsi évaluées sont budgétisées, c'est-à-dire qu'elles sont mises de côté dans le(s) budget(s) annuel(s) et à moyen terme, et que les partenaires du développement se sont engagés à fournir les ressources supplémentaires nécessaires.

Les coûts de mise en œuvre de la stratégie doivent être calculés à l'avance afin de prendre en compte les ressources financières nécessaires lors de l'établissement du CDMT et du (des) budget(s) annuel(s). Par ailleurs, lors de la rédaction d'un plan d'action pour une stratégie de RAP, l'institution chef de file et les autres institutions concernées devraient consulter le ministère des finances sur la marge de manœuvre budgétaire existante dans le CDMT, afin d'éviter d'être trop optimiste dès le début.

Dans l'idéal, lorsqu'une stratégie et son budget sont préparés, la mise en œuvre des actions envisagées doit prendre en compte le processus de préparation du budget annuel, afin de s'assurer qu'aucune action n'est planifiée dont la mise en œuvre pourrait être retardée car elle n'est pas prise en compte dans le budget annuel en cours ou à venir n'est planifiée, ou qu'aucune charge financière imprévue ne mette pas en danger le projet d'exécution du budget de l'État.

Idéalement, le chiffrage devrait utiliser des méthodes approuvées par le gouvernement ou le ministère des finances du pays. Les gouvernements peuvent appliquer diverses méthodes pour calculer les coûts des politiques existantes ou nouvelles (la réforme de l'administration publique représente un ensemble un ensemble de nouvelles politiques), comme par exemple le chiffrage des activités, le calcul du coût de revient unitaire ou le chiffrage de projet, par exemple. Souvent cependant, ces méthodes ne sont pas utilisées. C'est pourquoi pour les besoins de ce Manuel, l'expérience et le travail de SIGMA dans le soutien au chiffrage des stratégies de RAP dans divers pays ont été résumés et sont présentés dans les sections suivantes. L'approche, la terminologie et les détails du processus de chiffrage détaillés ci-après ne prétendent pas être exhaustifs ni représenter la meilleure approche possible, mais ils ont été développés en prenant en compte les 
concepts et les exigences définis dans les Principes, ainsi que le niveau de complexité et de qualité de chiffrage qui est raisonnablement attendu de la part des partenaires de SIGMA, compte tenu des compétences de leurs administrations ${ }^{42}$. L'Annexe 4 Partie I fournit un modèle de tableau de chiffrage. De plus, une partie de la Stratégie transversale de RAP 2015-2020 de l'Albanie est présentée en exemple dans l'Annexe 4 Partie II.

\subsection{Typologie des coûts, et quelques approches de calcul}

Les pays peuvent, bien sûr, choisir leur propre approche pour le chiffrage des politiques, notamment en ce qui concerne le chiffrage et la budgétisation des stratégies. Cependant, il existe certains concepts universellement admis, qui devraient être pris en compte pour décider de la méthode de chiffrage des stratégies. Lors du chiffrage des différentes actions, activités, services et réalisations d'une stratégie, il est nécessaire d'identifier le type de coûts induits par leur mise en œuvre. Dans la plupart des approches de chiffrage, les dépenses sont classées en coûts directs/indirects ou en coûts fixes/variables.

L'une des manières de préciser les coûts est d'identifier les coûts directs et indirects des activités, réalisations (ou produits) ou services. Les coûts directs sont des dépenses directement liées à l'exercice de fonctions ou à la fourniture de services. Ces coûts peuvent comprendre, notamment, les salaires, les matériaux et la formation. Les coûts indirects sont des dépenses découlant indirectement de l'action engagée (par exemple, la location de bureaux, les dépenses de maintenance et autres frais généraux). Ces dépenses sont à la charge d'un organisme chargé d'appliquer la stratégie, un ministère par exemple, dans le cadre de son mandat général, et sont par conséquent difficiles à rattacher à une activité particulière car elles en concernent plusieurs activités. Les coûts indirects ne peuvent être totalement ou facilement associés à des services ou réalisations spécifiques, mais sans eux, la fourniture de ces services serait impossible. Par exemple, dans le traitement des demandes de passeport des citoyens, les exemples de coûts directs peuvent comprendre :

- les salaires des fonctionnaires traitant les demandes de passeport et la production des passeports eux-mêmes

- l'achat des matériels utilisés pour fabriquer les passeports

- la formation des fonctionnaires pour traiter les demandes et fabriquer les passeports

Les exemples de coûts indirects peuvent être :

- l'utilisation de l'électricité, de l'eau et du chauffage

- les coûts téléphoniques et d'utilisation des TIC

On peut identifier les coûts d'une autre manière, en les structurant en coûts fixes et variables. Les coûts variables sont des dépenses qui varient selon les volumes produits, les activités et services fournis. Ils peuvent inclure les dépenses d'acquisition de matériaux, les coûts de communication, les coûts de formation, etc. Les coûts fixes sont constants et ne varient pas selon le volume de l'activité en question (par exemple: location des bureaux, coûts des équipements et frais généraux). En prenant le même

${ }^{42}$ Un guide exhaustif et détaillé pour le chiffrage des stratégies a été élaboré par la Regional School of Public Administration, disponible sur: http://www.respaweb.eu/11/library\#respapublications-2018-7. 
exemple du service de traitement des demandes et de délivrance des passeports des citoyens, on pourrait considérer comme coûts variables :

- l'achat des matériels utilisés pour fabriquer les passeports demandés

- la formation des fonctionnaires pour traiter les demandes et fabriquer les passeports

Des exemples de coûts fixes peuvent être :

- les salaires des fonctionnaires

- l'utilisation d'électricité, d'eau et de chauffage

- le coût des TIC et téléphones

La mise en œuvre d'une stratégie implique habituellement non pas un mais plusieurs organismes, soit, autrement dit, plusieurs programmes budgétaires. Lorsque la stratégie et son plan d'action sont élaborés, il est donc d'une importance capitale de connaître les conséquences budgétaires des actions prévues sur tous les budgets des organismes chargés de les mettre en œuvre.

En règle générale, lors du chiffrage d'une stratégie, il est plus important d'être clair sur les coûts directs ou variables encourus, car ils constituent habituellement une charge financière qui vient s'ajouter au budget de base initiale de l'organisme. Les salaires des fonctionnaires ne sont pris en compte que lorsque de nouvelles institutions doivent être créées, ou de nouveaux fonctionnaires recrutés, en conséquence d'une action envisagée. Les autres coûts fixes (qu'ils soient directs ou indirects), tels que les salaires des fonctionnaires déjà en poste, les équipements ou les téléphones, ne sont pas inclus, en règle générale, dans le chiffrage d'une stratégie, afin de ne pas trop compliquer le processus de chiffrage et réussir à élaborer la stratégie et de son plan d'action chiffré puisse se faire dans un délai convenable. Néanmoins, si l'administration du pays a des capacités et compétences de chiffrage plus sophistiquées, il peut être utile d'estimer tous les coûts de mise en œuvre de la stratégie. Dans ce cas tous les coûts, y compris les éléments de coût existants (c'est-à-dire en incluant tous les coûts indirects et les salaires des fonctionnaires préparant l'application de la stratégie) doivent être calculés. Cela s'appelle un chiffrage complet. Si cette méthode est appliquée, il faut faire particulièrement attention à ne pas chiffrer les éléments plusieurs fois (double comptage) et à rattacher proportionnellement les coûts fixes ou indirects aux activités de réforme avec le plus de précision possible.

On peut calculer les coûts totaux d'une action envisagée (par exemple, la formation des hauts fonctionnaires) en relevant le coût moyen par unité de production et en le multipliant par le nombre prévu de ces unités. Cependant, cette méthode ne peut être appliquée qu'à des services qui peuvent être standardisés (dans notre exemple, le coût unitaire pour l'activité envisagée serait le coût moyen de formation d'un fonctionnaire). Les calculs de coûts unitaires ne peuvent être entrepris pour tous les services connexes, car ils sont souvent dissemblables, et donc leur coût unitaire n'est pas standard. Par exemple, la création d'un nouveau système de rémunération dans la fonction publique, la numérisation des services publics, et la réalisation de consultations publiques conformes à la législation du pays ne peuvent être standardisées. Dans ces cas, on doit utiliser un chiffrage par activité ou par projet, plus sophistiqué. Ces approches affectent des coûts à une activité ou à un projet qui se concrétisent dans un ensemble de produits ou services.

D'une manière générale, l'approche recommandée pour le calcul du coût de mise en œuvre d'une stratégie est donc d'identifier les coûts directs additionnels pour chaque 
activité planifiée dans le plan d'action. Cependant, les coûts sont habituellement aussi classés selon leur catégorie économique, comme dans le plan comptable, qui est utilisé pour la préparation et la gestion du budget annuel du pays. Les grandes catégories économiques sont :

\section{Récurrent}

- biens et services

- voyages

- fournitures et dépenses de bureau

- intérêts des emprunts

- subventions

- autres transferts

- dépenses sociales

\section{Capital (non récurrent)}

- acquisition d'actifs immobiles

- remboursement de prêts

\section{2. Étapes logiques du processus de chiffrage}

Le chiffrage des éléments du plan d'action dans une stratégie implique plusieurs étapes, détaillées dans le Schéma 6. Cette description schématique ne présente que les principales étapes considérées comme essentielles pour parvenir à une estimation raisonnable de tous les éléments qui constituent le plan d'action de la stratégie. On pourrait inclure plus de détails, notamment les étapes préparatoires, mais nécessaires, liées à la planification du processus réel de chiffrage (responsabilités, délais, méthodes de calcul, identification des sources de données, etc.).

\section{Schéma 6. Étapes du processus de chiffrage}

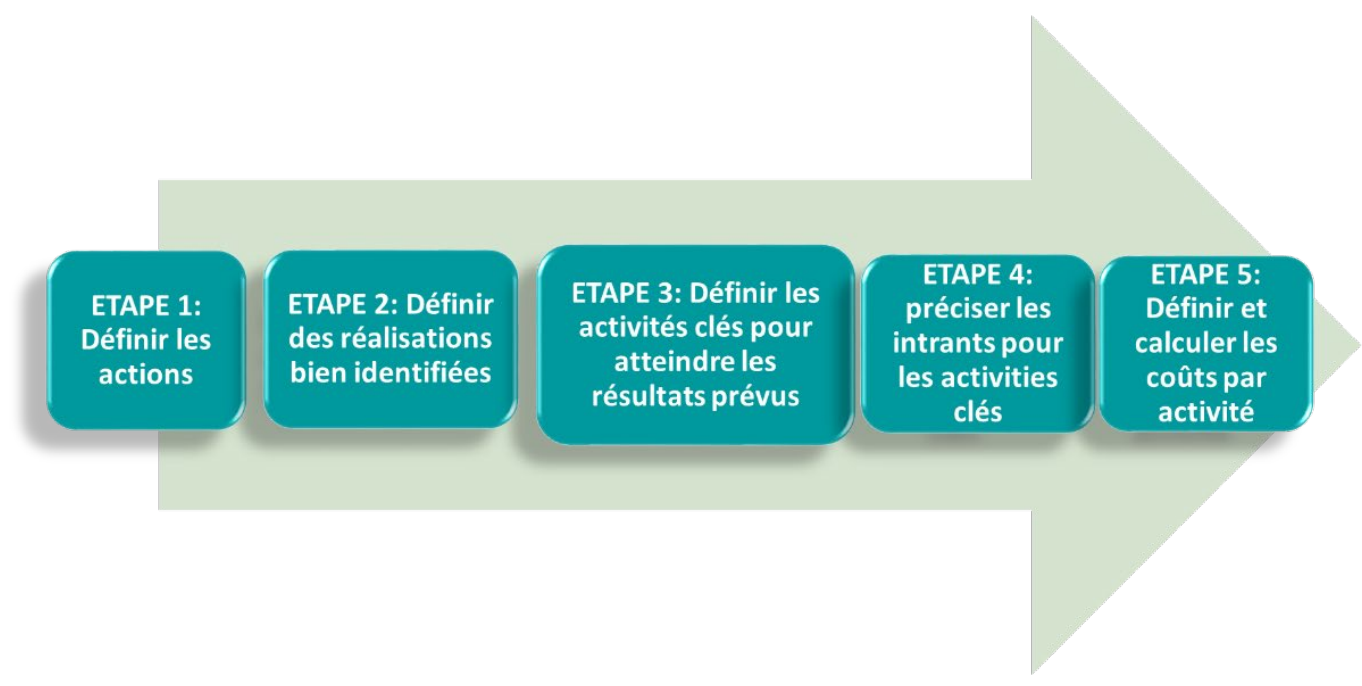

L'expérience pratique montre que, souvent, les plans d'action stratégiques des partenaires SIGMA ne vont pas plus loin que l'étape 2. Ils ne comprennent que les actions pour chaque objectif et leurs indicateurs respectifs de niveau de réalisation ou de processus. Ainsi, en pratique, peu de ces plans comprennent des chiffrages aussi détaillés. Afin de parvenir à une estimation globale plus précise des coûts de mise en 
œuvre de toutes les actions clés, il est nécessaire de décomposer les actions en activités chiffrables et intrants nécessaires. Les activités (c'est-à-dire tout type de mesure, programme, projet ou assimilé) sont les mesures les plus spécifiques qui doivent être prises afin de réaliser l'action et d'assurer ses résultats. Les intrants sont les ressources financières, humaines et matérielles nécessaires pour fournir ces résultats ou ces produits. La logique du chiffrage est présentée dans le Schéma 7. Il faut remarquer comment cette approche permet de construire graduellement le coût final de mise en œuvre de totalité de la stratégie, activité par activité, à partir de tous les chiffrages détaillés. Cette approche est également connue sous le nom de chiffrage ascendant.

\section{Schéma 7. Intrants utilisés pour le chiffrage}

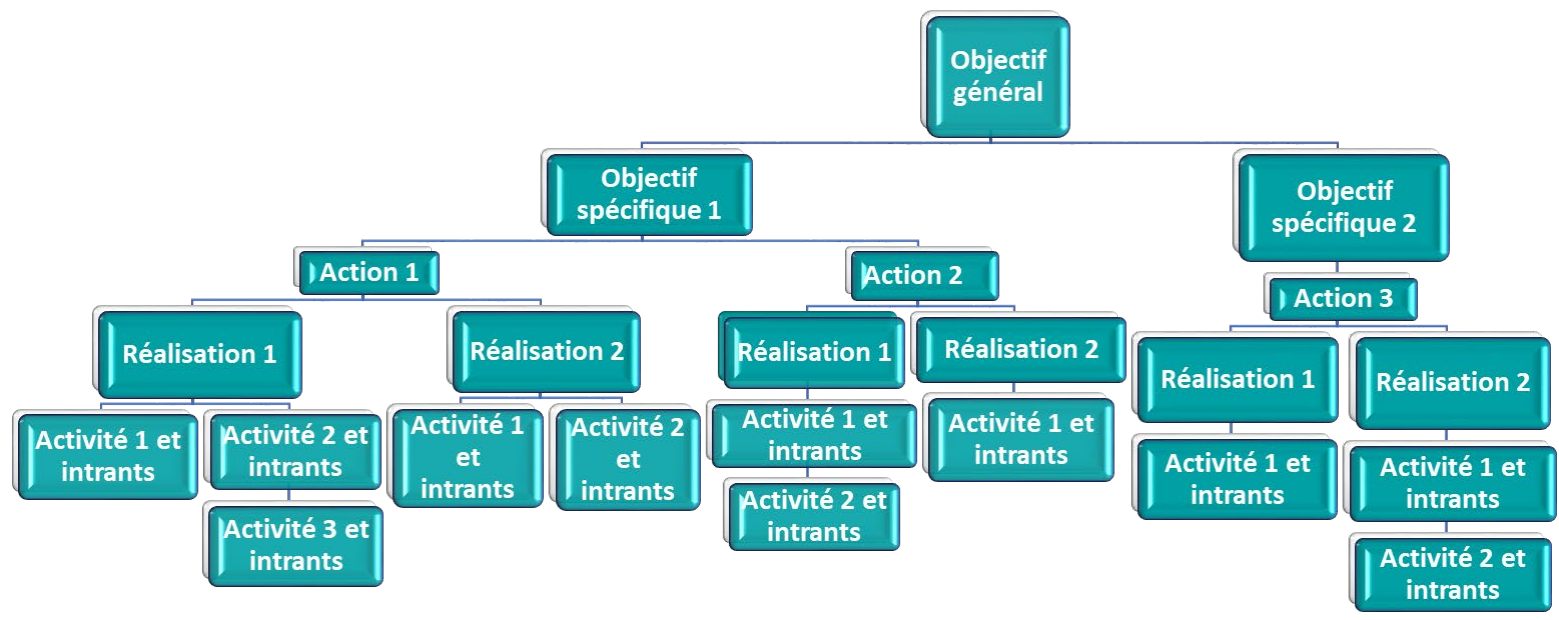

Les types les plus fréquents d'activités (et de leurs intrants nécessaires) sont les suivants :

- Mesures douces - assistance technique, activités d'amélioration des compétences (ateliers, formations, etc.), publications, campagnes de sensibilisation et d'information, etc.

- Investissement - achat ou développement de systèmes de TI, construction d'immeubles publics, achat d'équipement, etc.

- Ressources humaines - réorganisation ou recrutement de nouvelles personnes.

Afin de s'assurer que tous les éléments clés sont pris en compte et que les typologies et classifications nécessaires des différents éléments de chiffrage sont incluses, l'utilisation de feuilles de calcul Excel, ou de tout autre outil permettant facilement de faire des calculs et modifications, est fortement recommandée. Le Tableau 4 présente un exemple de la manière dont les actions envisagées sont segmentées en activités et leurs intrants.

Une fois que les activités et les intrants ont été définis (Étapes 3 et 4), il est nécessaire de définir des prix de référence et de calculer le budget requis (Étape 5). Cette étape 
implique de transformer les intrants en coûts financiers. Les activités et les intrants peuvent être transformés en coûts de plusieurs manières :

- En appliquant la méthode du coût unitaire moyen ;

- En appliquant les coûts historiques de services similaires (c'est-à-dire le même type de coûts pour des activités passées similaires);

- En appliquant les prix de référence moyens fournis par une enquête ou d'autres sources externes. Si les intrants sont des biens ou des services qui doivent être achetés auprès du secteur privé (par exemple, pour obtenir une étude de faisabilité, organiser une formation ou acheter de la publicité dans les médias sociaux), le coût de l'intrant en question sera connu en demandant des devis à plusieurs fournisseurs potentiels de services (peut-être par des demandes téléphoniques ou par email). Les devis peuvent ensuite servir à calculer un prix moyen, qui sera ensuite utilisé comme coût estimé de l'intrant concerné dans le processus de chiffrage de la stratégie.

En transformant des intrants en coûts financiers, il faut absolument prendre en compte la fréquence à laquelle ces coûts surviendront. Si le coût ne doit être assumé qu'une seule fois (par exemple, dans le cas d'un cycle de formation non renouvelé ou d'un investissement ponctuel dans le développement d'un système informatique), il s'agit d'un coût ponctuel. Mais s'il est nécessaire de recourir plusieurs fois à un intrant au cours de la mise en œuvre de l'activité en question, ou s'il faut y avoir de nouveau recours alors qu'une activité est mise en œuvre, il est considéré comme un coût récurrent. Si le coût doit survenir plusieurs années de suite, le coût total pour ces années doit être communiqué (en fournissant le montant annuel et le nombre d'années au cours ce coût surviendra). Le Tableau 4 fournit un exemple du chiffrage des activités et intrants.

Dans tous les cas, il est important de présenter tous les principaux éléments relatifs aux principaux intrants, que le calcul du coût final de mise en œuvre d'une activité donnée soit clairement retracé dans le tableau détaillé utilisé pour le chiffrage. Néanmoins, les détails d'un tel tableau (ou tableaux) de chiffrage peuvent être agrégés au niveau des activités, et peut-être même au niveau de chaque objectif retenu, afin de faciliter la présentation des coûts finaux des réformes aux décideurs et aux lecteurs externes de la stratégie. 
Tableau 4. Exemples d'actions théoriques liées à la RAP, avec les activités clés, les intrants et le chiffrage

\begin{tabular}{|c|c|c|c|c|c|}
\hline No. & Action & Réalisations & Activité & Intrants & Coûts \\
\hline \multirow[t]{3}{*}{1.} & \multirow{3}{*}{$\begin{array}{l}\text { Créer un centre } \\
\text { d'évaluation (CE) } \\
\text { pour la sélection, le } \\
\text { recrutement et } \\
\text { l'affectation des } \\
\text { hauts fonctionnaires, } \\
\text { fondé sur les } \\
\text { pratiques } \\
\text { internationales. }\end{array}$} & $\begin{array}{l}\text { Document } \\
\text { conceptuel } \\
\text { élaboré et adopté } \\
\text { avant T2 } 2019\end{array}$ & $\begin{array}{l}\text { Préparation du } \\
\text { document } \\
\text { conceptuel }\end{array}$ & $\begin{array}{l}50 \text { jours- } \\
\text { hommes }(\mathrm{JH}) \\
\text { d'assistance } \\
\text { technique }\end{array}$ & $\begin{array}{l}\text { Activité ponctuelle. Le } \\
\text { coût d'1 } \mathrm{JH} \text { est } 300 € \text {. } \\
50 \mathrm{JH} \times 300= \\
\mathbf{1 5 0 0 0} €\end{array}$ \\
\hline & & $\begin{array}{l}\text { Décret } \\
\text { gouvernemental } \\
\text { sur la création du } \\
\text { CE adopté avant } \\
\text { fin } 2019\end{array}$ & $\begin{array}{l}\text { Préparation du } \\
\text { Décret } \\
\text { gouvernemental }\end{array}$ & $\begin{array}{l}\text { Contribution } \\
\text { des } \\
\text { fonctionnaires } \\
\text { en poste }\end{array}$ & $\begin{array}{l}\text { Budget de base (pas de } \\
\text { coûts additionnels) }\end{array}$ \\
\hline & & $\begin{array}{l}\text { Le budget annuel } \\
2020 \text { inclut le } \\
\text { budget du CE }\end{array}$ & $\begin{array}{l}\text { Préparation du } \\
\text { budget annuel } \\
\text { avec le budget } \\
\text { du CE }\end{array}$ & $\begin{array}{l}\text { Contribution } \\
\text { des } \\
\text { fonctionnaires } \\
\text { en poste }\end{array}$ & $\begin{array}{l}\text { Budget de base (pas de } \\
\text { coûts additionnels) }\end{array}$ \\
\hline \multirow[t]{3}{*}{2.} & \multirow{3}{*}{$\begin{array}{l}\text { Développer et mettre } \\
\text { en ouvre un } \\
\text { nouveau Programme } \\
\text { de perfectionnement } \\
\text { des cadres (PPC) } \\
\text { pour les cadres } \\
\text { supérieurs. }\end{array}$} & \multirow{2}{*}{$\begin{array}{l}\text { Décret } \\
\text { ministériel sur le } \\
\text { Programme de } \\
\text { perfectionnement } \\
\text { des cadres (PPC) } \\
\text { adopté avant T2 } \\
2020\end{array}$} & $\begin{array}{l}\text { Préparation du } \\
\text { PPC }\end{array}$ & $\begin{array}{l}20 \mathrm{JH} \\
\text { d'assistance } \\
\text { technique }\end{array}$ & $\begin{array}{l}\text { Activité ponctuelle. Le } \\
\text { coût d'1 JH est } 300 € \text {. } \\
20 \mathrm{JH} \text { x } 300=6 \mathbf{0 0 0} €\end{array}$ \\
\hline & & & $\begin{array}{l}\text { Préparation du } \\
\text { décret du } \\
\text { ministre de } \\
\text { l'Administration } \\
\text { publique }\end{array}$ & $\begin{array}{l}\text { Contribution } \\
\text { des } \\
\text { fonctionnaires } \\
\text { en poste }\end{array}$ & $\begin{array}{l}\text { Budget de base (pas de } \\
\text { coûts additionnels) }\end{array}$ \\
\hline & & $\begin{array}{l}\text { Compétences de } \\
\text { leadership } \\
\text { accrues des hauts } \\
\text { fonctionnaires } \\
\text { lorsque } 40 \% \text { des } \\
\text { hauts } \\
\text { fonctionnaires } \\
\text { auront suivi le } \\
\text { PPC avant fin } \\
2022\end{array}$ & $\begin{array}{l}\text { Formation de } \\
120 \text { hauts } \\
\text { fonctionnaires }\end{array}$ & 15 formateurs & $\begin{array}{l}\text { La formation d'un haut } \\
\text { fonctionnaire coûte } \\
700 € \text { (tout compris). } \\
\text { L'activité sera menée } \\
\text { chaque année pour } 120 \\
\text { fonctionnaires. } 120 \times \\
700 \times 3 \text { (plan d'action } \\
\text { sur } 3 \text { ans) = } \mathbf{2 5 2} \mathbf{0 0 0} €\end{array}$ \\
\hline \multirow[t]{4}{*}{3.} & \multirow{4}{*}{$\begin{array}{l}\text { Mettre à jour le } \\
\text { Registre de la } \\
\text { fonction publique } \\
\text { pour recueillir et } \\
\text { analyser des données } \\
\text { sur la gestion et le } \\
\text { développement de la } \\
\text { haute fonction } \\
\text { publique. }\end{array}$} & \multirow{4}{*}{$\begin{array}{l}\text { Création d'une } \\
\text { fonction pour } \\
\text { recueillir et } \\
\text { analyser des } \\
\text { données sur la } \\
\text { gestion et le } \\
\text { développement } \\
\text { des hauts } \\
\text { fonctionnaires }\end{array}$} & $\begin{array}{l}\text { Conception du } \\
\text { périmètre de la } \\
\text { fonction TI }\end{array}$ & $\begin{array}{l}5 \mathrm{JH} \\
\text { d'assistance } \\
\text { technique } \\
\text { (contenus) }\end{array}$ & $\begin{array}{l}\text { Activité ponctuelle. Le } \\
\text { coût d'1 JH est } 300 € .5 \\
\text { JH x } 300=\mathbf{1 5 0 0} €\end{array}$ \\
\hline & & & $\begin{array}{l}\text { Développement } \\
\text { de la } \\
\text { fonctionnalité } \\
\text { des TI }\end{array}$ & $\begin{array}{l}40 \mathrm{JH} \\
\text { d'assistance } \\
\text { technique } \\
\text { (logiciel) }\end{array}$ & $\begin{array}{l}\text { Activité ponctuelle. Le } \\
\text { coût d'1 JH est } 300 € \text {. } \\
40 \mathrm{JH} \text { x } 300=\mathbf{1 2 0 0 0} €\end{array}$ \\
\hline & & & $\begin{array}{l}\text { Formation de } 10 \\
\text { personnels RH } \\
\text { du ministère de } \\
\text { l'Administration } \\
\text { publique }\end{array}$ & 10 formateurs & $\begin{array}{l}\text { La formation d'un } \\
\text { personnel RH coûte } \\
100 € \text {. Activité } \\
\text { ponctuelle pour } 10 \\
\text { employés. } 10 \times 100= \\
1000 €\end{array}$ \\
\hline & & & $\begin{array}{l}\text { Recueil des } \\
\text { données } \\
\text { nécessaires }\end{array}$ & $\begin{array}{l}\text { Contribution } \\
\text { des } \\
\text { fonctionnaires } \\
\text { existants }\end{array}$ & $\begin{array}{l}\text { Budget de base (pas de } \\
\text { coût additionnel) }\end{array}$ \\
\hline & \multicolumn{4}{|c|}{ Coût total des actions $1-3$ pour la durée prévue du Plan d'action : } & $286000 €$ \\
\hline
\end{tabular}


Note 1. Ce calcul est fondé sur les prix du marché lorsque le service est totalement externalisé. Cependant, dans certains cas, les organismes peuvent organiser des formations en engageant des experts en formation et en prenant des dispositions logistiques propres. Dans ce cas, il faudra calculer les coûts des intrants suivants : coût du formateur, du matériel de formation, de la location du salle, de la restauration, de l'hébergement, etc.

Note 2. Les coûts de formation des différents groupes cibles ont tendance à varier, et le font souvent. Par exemple, la formation d'un haut fonctionnaire peut être beaucoup plus chère, car cette formation impliquera des approches plus individuelles, telles que le coaching ou le mentorat.

\subsection{Répercussion des résultats du chiffrage avant la finalisation de l'élaboration de la stratégie}

Une fois que les coûts de mise en œuvre de la stratégie ont été déterminés, il ne faut pas négliger la tâche subsidiaire qui consiste à en évaluer la faisabilité financière par rapport au cadre de dépenses à moyen terme du pays et à son budget annuel en cours ou à venir. Une stratégie peut contenir à la fois des activités nouvelles et d'anciennes activités entamées sous de précédentes réformes. C'est pourquoi il est important, pour chaque activité envisagée, de savoir si, et dans quelle mesure, les coûts identifiés sont inclus dans le budget, c'est-à-dire déjà pris en compte en tant que coûts prévisibles (comme dans le cas, par exemple, des salaires des fonctionnaires en poste qui réaliseront l'activité prévue), ce qui n'occasionnera pas de dépenses supplémentaires, ou si l'activité en question est une initiative entièrement nouvelle nécessitant un complément de financement s'ajoutant aux ressources dont dispose déjà les institutions chargées de la mettre en œuvre. Les initiatives nouvelles peuvent exiger des financements additionnels, qu'il faut apprécier à l'aune des plafonds de dépenses globaux et par institution.

Si la mise en œuvre de la stratégie envisagée exige des ressources additionnelles et que les coûts identifiés excèdent le(s) plafond(s) actuel(s), le gouvernement du pays devrait, sur la base des recommandations de l'institution chef de file (décidées en collaboration étroite avec le ministère des finances), donner de nouvelles priorités d'initiatives de dépenses de la (les) manière(s) suivante(s) :

- réaffectation de fonds de domaines ou programmes à faible priorité vers des domaines/programmes à haute priorité

- réévaluation des objectifs et cibles qui étaient initialement fixés

- réajustement de la liste de priorités de la stratégie, en éliminant les initiatives de faible priorité

- réduction de l'envergure de la stratégie ou élimination de certaines initiatives

- reprogrammation de la mise en œuvre de certaines initiatives de mesures

- élimination de nouvelles initiatives de dépenses en trouvant, par exemple, d'autres moyens, moins exigeants en ressources, d'atteindre les objectifs

- recherche d'autres sources de financement telles que des partenaires de développement ou des emprunts

Si les ressources sont inférieures à ce qui est nécessaire pour accomplir le but des réformes, il vaut mieux en premier lieu chercher des solutions alternatives internes pour réaliser les activités prévues, en réaffectant des fonds de programmes à faible priorité vers des domaines à plus haute priorité, en faisant des gains d'efficience, etc. S'il est impossible de trouver des financements provenant de ressources disponibles dans le cadre des budgets institutionnels de base ou des programmes fixés pour les initiatives prévues, il est alors recommandé de faire appel à d'autres sources externes, 
partenaires du développement, bailleurs de fonds, institutions financières internationales ou des emprunts.

En général, lorsqu'on compare le niveau de ressources nécessaires pour les réformes au financement disponible, il est important de calculer avec prudence, afin de s'assurer que ce qui reste dans la stratégie peut réellement être mis en œuvre, et que le plan d'action envisagé, en termes de délais et de résultats souhaitées, n'est pas freiné par un manque de financement. Dans ce but, l'institution chef de file et les organes de mise en œuvre responsables de la définition des actions de la stratégie et de l'élaboration des activités, devraient étroitement collaborer avec le ministère des Finances pendant toute la durée du processus de finalisation de la stratégie et de son plan d'action.

Néanmoins, les stratégies et leurs plans d'action correspondant fonctionnent souvent encore malgré des insuffisances de financement, tant que ces insuffisances font l'objet de débats approfondis et qu'elles sont constamment suivies et gérées afin que soient régulièrement appréciées (dans l'idéal, chaque année, conformément au cycle du budget annuel) les ressources nécessaires et la disponibilité des fonds.

Il est également important que toutes les institutions responsables de la mise en œuvre de la stratégie envisagée utilisent les informations concernant les coûts (ainsi que d'autres informations sur la stratégie, notamment sur les cibles des indicateurs) pour transmettre leurs demandes budgétaires au ministère des Finances, en respectant le format et les obligations de la planification budgétaire du pays concerné.

\section{Réflexions pratiques :}

$\checkmark$ On suppose souvent que le chiffrage exige une méthodologie sophistiquée, alors qu'en fait il ne requiert que l'application précise de certains concepts et méthodes essentiels.

$\checkmark$ Le chiffrage peut exiger de voir au-delà des actions. Les rédacteurs doivent pleinement appréhender ce qui est nécessaire à la mise en ouvre de l'action, c'est pourquoi il est généralement nécessaire de décomposer l'action en plus petites étapes, c'est-à-dire en activités clés.

$\checkmark$ Le chiffrage doit tenir compte des obligations nationales existantes et doit être mené dans le cadre d'un processus itératif, impliquant toutes les institutions qui peuvent avoir un rôle à jouer dans la mise en ouvre des actions envisagées, et en étroite collaboration avec le ministère des Finances du pays.

$\checkmark$ En général, la qualité du chiffrage dépend de la qualité des plans. Plus le plan d'action est clair à propos des activités envisagées, mieux leur coût pourra être calculé.

Ne pas oublier :

$\checkmark$ Le chiffrage d'une stratégie est basé sur son plan d'action, et il s'agit d'un processus logique qui doit être inclusif et itératif.

$\checkmark$ Les actions contenues dans le plan d'action doivent souvent être décomposées et détaillées pour arriver à une estimation solide des ressources financières requises pour leur mise en cuvre.

$\checkmark$ Lors de l'estimation du coût de mise en cuvre d'une stratégie, il faut prendre en compte à la fois les coûts des activités déjà en cours pour lesquelles des 
financements additionnels ont été obtenus, et les coûts estimés des nouvelles activités.

$\checkmark$ À moins que le gouvernement n'exige un chiffrage complet, le coût des salaires de fonctionnaires déjà en poste dans les institutions chargées de la mise en æuvre n'est habituellement pas pris en compte dans le calcul, ces salaires faisant déjà partie $d u$ budget de base. Seuls les salaires concernant de nouvelles institutions ou du personnel supplémentaire sont calculés et doivent être reflétés dans les budgets annuels du pays ainsi que dans son CDMT.

$\checkmark$ Étant donné que les ressources disponibles sont habituellement limitées, les résultats de l'exercice de chiffrage doivent être répercutés dans le processus de planification, pour permettre la réaffectation des ressources, le réajustement des priorités ou la redéfinition de ce qui peut être accompli de manière réaliste avec le financement disponible, et quand. Les coûts additionnels devraient d'abord être financés par une réaffectation interne et des économies, et les demandes de soutien externes ne devraient être envisagées qu'en deuxième option.

$\checkmark$ Il n'existe pas de méthode unique, correcte et universellement applicable pour le chiffrage d'une stratégie, les méthodes et approches peuvent varier. Cependant, quelle que soit l'approche choisie, elle doit être appliquée de manière systématique et cohérente tout au long de l'exercice. On peut aussi utiliser une combinaison de méthodes, mais leur application doit tout de même être cohérente. 


\section{Suivi et reporting (rédaction de rapports)}

Après qu'une stratégie et son plan d'action ont été approuvés, la mise en œuvre débute. Les institutions doivent constamment et continuellement en suivre et en évaluer les progrès ${ }^{43}$, pour voir si la mise en œuvre de la stratégie permet d'atteindre ses objectifs et tient ses promesses ${ }^{44}$. Deux étapes aident à évaluer le succès de la stratégie et de sa mise en œuvre :

- Le suivi et le reporting

- L'évaluation

Le suivi, le reporting et l'évaluation d'une stratégie sont indispensables, mais constituent des étapes du processus de stratégie très exigeantes en termes de ressources et de moyens. Le but principal des efforts de suivi et de reporting est d'identifier les problèmes et de faire des recommandations pour les résoudre pendant la mise en œuvre de la stratégie. Il est d'une importance capitale que le système de suivi, de reporting et d'évaluation soit développé et arrêté en même temps qu'est adoptée la stratégie, et détaille les différents rôles et responsabilités concernant la fourniture et le recueil de données, la rédaction des rapports, la fréquence du reporting, la structure des différents rapports, les principaux aspects de l'évaluation, etc. Si le cadre d'un pays est constitué de différentes stratégies, il est également important de faire en sorte que le processus de suivi et de reporting soit standardisé et harmonisé, afin de réduire la charge supplémentaire relevant de chacune des stratégies du cadre. Il est aussi important que tout système de suivi, de reporting et d'évaluation soit conforme aux exigences et normes du système national, lorsqu'elles existent.

Le plus difficile est de déterminer comment recueillir, analyser et présenter (ainsi que comment communiquer) l'information de la meilleure façon possible afin qu'elle permette l'amélioration des politiques et la prise de décision, et qu'elle éclaire les usagers externes et le public au sens large. Trouver cet équilibre délicat requiert les efforts constants, l'expertise et les compétences de la part du personnel technique, comme des dirigeants et décideurs qui soutiennent la démarche. Plusieurs cycles de reporting sont nécessaires pour correctement et finement déterminer quelle information est requise, quand et par qui, et d'apporter ainsi le meilleur soutien possible à une mise en œuvre harmonieuse et efficace.

L'élaboration complète d'un système de suivi, de reporting et d'évaluation peut, en pratique, prendre plusieurs années avant de pouvoir servir pleinement et efficacement

${ }^{43}$ Pour plus d'informations sur le suivi de la performance administrative, voir OCDE (2009), Measuring Government Activity, Éditions Oaris, https://doi.org/10.1787/9789264060784-en.

44 Pour plus d'informations sur le reporting, voir: https://ec.europa.eu/neighbourhoodenlargement/tenders/monitoring-and-evaluation cs. En outre, l'édition 2017 de Quality of Public Administration - Toolbox de la Commission européenne peut être un outil utile. Ce manuel souligne l'implication active de parties prenantes dans l'évaluation des politiques publiques, et fournit aussi des exemples d'approches participatives pour le suivi et l'évaluation, impliquant toutes les parties prenantes concernées. Voir sur : http://ec.europa.eu/social/main.jsp? catId=738\&langId=en\&pubId=8055\&type=2\&furtherPubs= $\underline{\text { no }}$ 
les objectifs des décideurs et de devenir un ensemble d'outils couramment utilisés pour tenir les responsables de la réforme comptables des progrès des réformes. La mise en place d'un tel système commence souvent par la rédaction de simples rapports d'activité sur la mise en œuvre des actions, et il est amélioré au cours du temps avec l'augmentation de la précision et de la qualité de l'information obtenue au cours du processus, et selon la manière dont cette information est utilisée dans la prise de décisions. C'est pourquoi le développement d'un système exhaustif prend du temps et relève d'un processus itératif d'apprentissage par la pratique.

En fin de compte, il est utile d'avoir un système de suivi, de reporting et d'évaluation si celui-ci est opportun, ciblé, pertinent et convivial. L'Encadré 16 explique ce qui est entendu par ces termes.

\begin{tabular}{|l|l|}
\hline \multicolumn{2}{|c|}{ Encadré 16. Caractéristiques principales d'un bon système de suivi, } \\
de reporting et d'évaluation
\end{tabular}


Signifie que seules les informations pertinentes et stratégiques sont fournies dans les rapports. Les informations sur les affaires courantes et d'ordre administratif doivent être évitées, car elles surchargent les lecteurs, qui doivent habituellement traiter de nombreux sujets importants, et ne peuvent donc assimiler que des informations brèves, ciblées et concises, qui les aident à accomplir leur travail de gestion de la mise en ouvre de la stratégie et de prise de décisions politiques importantes.

Pertinent

Signifie que les informations sont fournies en utilisant une structure

Convivial simple, un texte concis et autant d'informations visuelles que possible. La langue doit également être simple.

\subsection{Le rôle du suivi, du reporting et de l'évaluation dans le processus stratégique}

Le suivi, le reporting et l'évaluation de la mise en œuvre et du succès de toute stratégie font partie intégrante du cycle de politique stratégique dans son ensemble, et jouent un rôle significatif dans l'information de toutes les parties prenantes (à l'intérieur comme à l'extérieur de l'administration du pays) sur la réalisation des engagements annoncés dans la stratégie et dans son plan d'action. Plus important encore, ils garantissent un retour d'informations aux gestionnaires et aux dirigeants politiques sur des questions politiques distinctes, ainsi que sur le progrès général de la mise en œuvre de la stratégie (y compris sur l'utilisation et la disponibilité des ressources financières nécessaires). L'utilisation du système de suivi, de reporting et d'évaluation permet des prises de décisions budgétaires et de politique stratégique avisées, et son emploi régulier et systématique fournit une bonne base pour prendre des décisions politiques cruciales au bon moment, en disposant de la meilleure information possible.

Le suivi, le reporting et l'évaluation aident à identifier et à transmettre les informations sur les défis et les goulets d'étranglement apparus lors de la mise en œuvre. Cette connaissance peut être utilisée pour concevoir et proposer des solutions d'amélioration de la stratégie et de son plan opérationnel, pour surmonter des difficultés spécifiques liées à sa mise en œuvre, et pour faire meilleur usage des structures de gestion et de coordination existantes. Autrement dit, un bon usage du système de suivi, de reporting et d'évaluation est fondamental pour atteindre les résultats prévus de la réforme en temps voulu, de manière efficace et efficiente. De plus, le suivi, le reporting et l'évaluation sont aussi liés à l'obligation de rendre compte. Les informations et données générées par le suivi et le reporting aident à tenir les institutions publiques responsables des engagements pris et reflétés dans les stratégies. Il est crucial que les résultats du processus de suivi, ainsi que toute évaluation, soient partagés avec les parties prenantes et le public dans son ensemble, pour garantir que tous les acteurs concernés sont informés de ce qui a progressé ou non, comment et avec quels résultats, ainsi que pour en valider les principales conclusions. Dans l'idéal, les parties prenantes (et les citoyens) sont impliquées dans le processus de suivi et d'évaluation en tant qu'apporteurs d'informations et influenceurs potentiels, avant toute prise de décision liées aux réformes proposées.

Les façons de réaliser le suivi et le reporting, ainsi que les méthodes utilisées pour leur élaboration et leur utilisation, sont significativement différentes de celles utilisées pour 
l'évaluation, bien que les bénéfices finaux soient similaires (prise de décisions informée et élaboration des politiques améliorée).

\subsection{Qu'est-ce que le suivi et le reporting?}

Le suivi est un processus régulier et récurrent, par lequel le personnel responsable recueille et analyse les informations sur l'état d'avancement d'une réforme à un moment donné (et au cours du temps), en ce qui concerne les cibles respectives de la réforme au niveau des résultats et des réalisations. Le suivi prend fin au niveau des réalisations, car il a lieu pendant la mise en œuvre des réformes, et l'obtention des impacts désirés ne peut être mesurée que par l'évaluation.

Sur la base des informations recueillies pendant le processus de suivi, des rapports de suivi sont rédigés pour informer les responsables de la mise en œuvre, les principaux décideurs et les autres parties prenantes, ainsi que le public , des progrès des réformes, des défis identifiés et des solutions proposées pour assurer le succès final des réformes. De bons rapports aident les décideurs à prendre les actions correctives qui permettront une mise en œuvre efficace et une meilleure conception des politiques.

La rédaction d'un bon rapport exige différents types de compétences et de capacités :

- compétences analytiques pour examiner et analyser les données, et formuler des jugements ou conclusions ;

- pensée créative pour structurer le rapport de manière conviviale ;

- capacités de communication pour communiquer les informations de performance de façon à intéresser les différents lecteurs.

La rédaction de rapport ne devrait pas être une activité routinière, mais un exercice renouvelé à chaque fois. Même si le recueil de données est répétitif et se fonde sur des indicateurs et des informations sur certaines activités qui ne changent pas d'une année sur l'autre, les rédacteurs devraient, pendant l'écriture du rapport lui-même, rechercher des innovations et des améliorations en termes de structure, d'aides visuelles, de description des défis et de proposition de solutions. En pratique, les rapports de suivi de stratégie sont trop souvent écrits en utilisant la même structure narrative en mettant simplement à jour les données et informations. Cette pratique devrait être évitée ou n'être utilisée qu'avec prudence afin de garder une approche ciblée et d'éviter de lasser des lecteurs de rapports récurrents.

\subsection{Quels sont les facteurs d'un bon suivi et d'un bon reporting ?}

Arriver à un bon résultat de suivi et de reporting exige davantage qu'une bonne stratégie et qu'un bon plan d'action. Trois questions principales déterminent le succès et l'efficacité du système de suivi et de reporting, comme il est montré dans le Schéma 8.

Schéma 8. Questions déterminant un reporting de bonne qualité
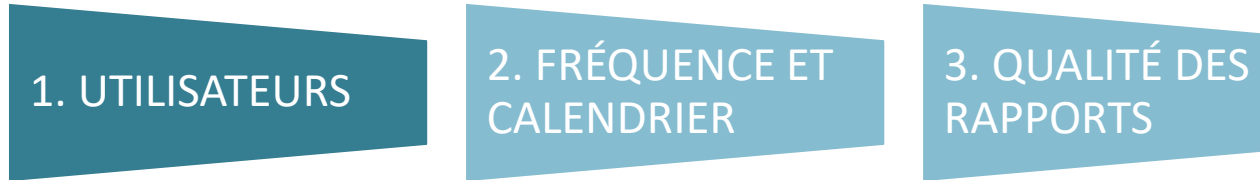


\subsubsection{Utilisateurs}

En premier lieu, le type d'informations fournies pour le suivi peut dépendre des utilisateurs de ces informations. Plus ceux-ci sont à un niveau de prise de décisions élevé, plus l'information devra être agrégée et axée sur les réalisations. Le rôle de ces lecteurs est de prendre des décisions stratégiques ; l'information qui leur est fournie doit donc être ciblée et stratégique. Mais plus le rapport est destiné à un personnel de niveau subalterne, plus il devra offrir d'informations plus opérationnelles.

Les utilisateurs habituels des informations de suivi et de reporting sont :

- Les utilisateurs externes : citoyens, médias, ONG, organismes professionnels, experts, universités, bailleurs de fonds, etc.

- Les utilisateurs internes: parlement, gouvernement, ministres, cadres et personnel opérationnel

En règle générale, les utilisateurs externes et les lecteurs internes de haut niveau tels que les membres du parlement, le gouvernement et les ministres (utilisateurs-type de la stratégie) sont davantage intéressés par les informations stratégiques concernant :

- le progrès global par rapport aux objectifs et cibles fixés pour les domaines clés (généralement les principaux piliers par lesquels ces domaines sont couverts dans la stratégie), notamment le montant des dépenses pour un niveau particulier de résultat;

- les défis systémiques rencontrés ;

- l'utilisation des ressources attribuées, en particulier utilisation du budget.

Les gestionnaires et le personnel opérationnel (les utilisateurs opérationnels) ont besoin d'informations stratégiques et opérationnelles (par exemple, sur des problèmes spécifiques de mise en œuvre, même occasionnels, ou sur des retards dus à des difficultés opérationnelles). Il est donc important que les rapports sur la mise en œuvre d'une stratégie et de son plan d'action comprennent à la fois des informations stratégiques et opérationnelles, présentées de Les utilisateurs des rapports de suivi :

$\checkmark$ Comprennent des utilisateurs stratégiques et opérationnels

$\checkmark \quad$ Ont besoin de différents types d'informations

$\checkmark \quad$ Exigent que toutes les informations de suivi contenues dans les rapports soient présentées de manière à ce qu'ils puissent facilement trouver ce dont ils ont besoin

manière à ce que chaque groupe cible puisse aisément trouver les informations correspondant à ses besoins.

\subsubsection{Fréquence et calendrier}

Le suivi est une activité de routine, et les données de suivi sont habituellement recueillies à une fréquence prédéfinie et spécifique pour l'établissement des rapports. La fréquence varie selon le type d'information sur la performance. Par exemple, les indicateurs de résultats (efficacité de l'administration, renouvellement des fonctionnaires, etc.) sont mesurés tous les ans ou même moins souvent, tandis que les indicateurs de production (nombre de fonctionnaires formés, nombre de consultations menées, etc.) sont mesurés et décrits plus souvent, par exemple chaque trimestre ou dès qu'approche l'échéance de remise du rapport.

La préparation des rapports est une activité qui demande beaucoup de travail, et elle devrait être faite de manière à ce que le résultat final (le rapport) soit proportionné en 
termes de coûts (le temps passé à son élaboration) et de bénéfices (l'utilisation réelle du rapport). En se fondant sur la pratique de plusieurs partenaires SIGMA dans les Balkans occidentaux et sur les besoins des décideurs et des partenaires du développement (en particulier l'UE), il est recommandé que le rapport couvre :

1. Les objectifs de stratégie et les indicateurs de résultat chaque année ou tous les deux ans.

2. Les activités du plan d'action de la stratégie et les indicateurs de production tous les six mois.

\subsubsection{Qualité des rapports}

Les rapports ne servent pas uniquement à obtenir des compte-rendus sur la mise en œuvre de la réforme et de la visibilité sur sa progression. Les rapports de stratégie permettent également de :

1. Prendre des décisions pour résoudre les problèmes et accélérer la mise en œuvre de réformes et d'activités à la traîne ou différées. Dans ce but, les rapports doivent comprendre un compte rendu des principaux goulets d'étranglement et défis, en se concentrant sur les problèmes systémiques au niveau stratégique (ainsi que sur les mesures correctives proposées pour les résoudre), tout en incluant des informations sur les difficultés spécifiques, parfois même ponctuelles, au niveau opérationnel. Les principaux goulets d'étranglement et défis peuvent provenir d'une absence de financement, à des procédures de marchés publics, de désaccords entre institutions, etc.

2. Chercher de meilleurs moyens d'atteindre les objectifs, non seulement par le recueil et l'analyse de données pour la rédaction des rapports, mais aussi par la synergie entre différentes perspectives et idées, qui peut être trouvée grâce à des discussions de haut niveau entre responsables clés du gouvernement et partie prenantes externes. Pour soutenir ces objectifs d'amélioration de l'élaboration des politiques, les rapports de stratégie devraient être présentés de manière attrayante, et d'une façon qui stimule la réflexion stratégique.

L'atteinte de ces objectifs de suivi et de reporting dépend de la compétences analytiques et des aptitudes créatives des fonctionnaires pour produire des rapports stratégiques et non simplement descriptifs, et pour les rendre accessibles, contrairement à l'habitude qui consiste à présenter l'ensemble des informations sans les avoir triées ni structurées, ou bien sous la forme des diapositives saturées de texte. En règle générale, on peut affirmer que la qualité des discussions et des décisions sur les questions relatives à la RAP ou au secteur concerné dépend en grande partie de la qualité et de la présentation des rapports qui les étayent.

Figurent ci-après certains des principaux problèmes des rapports. Ils sont :

- trop longs et seulement ciblés sur la fourniture descriptive d'informations administratives ;

- pauvres en réflexion analytique et en analyse des causes des mauvaises performances ou des goulets d'étranglement;

- peu agréables à lire, avec une structure complexe et peu d'illustrations. 
Encadré 17. Exemple lié à la RAP d'une réflexion sur les processus par opposition à une réflexion sur les résultats

Descriptions de processus $\quad$ Description de résultats

Le groupe de travail sur l'optimisation du secteur public s'est réuni quatre fois en 2015. Il a préparé une Feuille de route pour l'optimisation du secteur public. Le plan d'action a donné lieu à une consultation avec tous les ministères, les principales agences gouvernementales et organisations de la société civile. Le gouvernement a adopté la Feuille de route en septembre 2015. La Feuille de route couvre une période de trois ans et énumère de nombreuses activités prévoyant la fusion ou l'examen des fonctions.
La Feuille de route pour l'optimisation a permis de réduire les fonctions de soutien du secteur public de $10 \%$ dans les agences pendant les deux premières années de sa mise en œuvre. On estime qu'une réduction de $20 \%$ sera atteinte à la fin de l'année 2019. Pendant les deux premières années, le budget des fonctions de soutien a été réduit de 2,5 millions $€$. On estime que 5 millions $€$ auront été économisés à la fin de l'année 2018.

Un bon rapport de suivi de stratégie devrait donc être :

- Agréable et facile à lire. La présentation créative des données et de l'information par l'utilisation de graphiques, de dessins, de tableaux et d'autres outils modernes de présentation utilisés pour la communication stratégique se répand.

- Court et bien structuré. Personne ne lira un compte rendu long et descriptif des activités de mise en œuvre, mais la plupart des gens feuilletteront un court document ayant des titres courts et attrayants, quelques listes à puces, et des nombreux graphiques et tableaux. Les informations additionnelles et les justifications plus longues peuvent si besoin être présentées en annexe. Cette façon de présenter les rapports a de meilleures chances d'attirer l'attention des décideurs.

- Axé sur les résultats. Les aspects les plus importants sont ce qui a changé dans un domaine d'action particulier (c'est-à-dire l'impact depuis l'adoption de la stratégie ou depuis le dernier rapport), à quel point les engagements prévus ont été remplis au moment de la rédaction du rapport, ce qui n'a pas bien fonctionné et pourquoi, et ce qui doit être changé et comment (c'est-à-dire les recommandations pour des mesures ou actions correctives).

- Factuel. Les commentaires contenus dans le rapport doivent se fonder sur des faits, des informations vérifiées et des preuves valides.

- Analytique. Les rapports doivent être écrits de manière à aider les lecteurs à tirer leurs propres conclusions et à décrire significativement les résultats obtenus, mais plus particulièrement les difficultés et leurs causes sous-jacentes. 


\section{Encadré 18. Exemple d'un bon rapport de suivi}

\section{Optimisation de l'administration publique}

\section{Progrès :}

L'ambition principale en 2016 était de réduire le nombre de fonctionnaires et d'institutions conformément aux cibles $\mathrm{du}$ programme d'optimisation $\mathrm{du}$ gouvernement. L'atteinte de ces deux cibles a été retardée à cause de cinq lois en attente d'adoption au Parlement. Les lois devraient être adoptées lors de la prochaine session, ce qui permettra d'atteindre les cibles.

De plus, sous la coordination du ministère des Finances, tous les ministères ont œuvré à la mise en place de centres de services partagés pour 15 services. Cependant, cette cible n'a pas été atteinte, car $30 \%$ des institutions n'ont pas fourni d'informations sur trois principaux services, à savoir les marchés publics, la gestion documentaire, et la comptabilité.

\section{Recommandations :}

Les membres du gouvernement devraient promouvoir l'adoption des projets de loi en attente au Parlement.

Les institutions mentionnées dans le rapport devraient fournir l'information nécessaire pour achever la création des services partagés dans un délai de 3 semaines. Le règlement rapide de cette tâche devrait recevoir tout le soutien possible.

Les rapports de stratégie devraient être des témoignages sur la performance, s'appuyant sur des preuves. Pour élaborer de tels récits, le filtrage ou le tri des informations pertinentes est crucial. Au cours du suivi et du recueil de données, une grande quantité de détails est réunie. Les fonctionnaires responsables de la rédaction du rapport doivent pouvoir juger quels types d'informations ou de données doivent être utilisés et sous quelle forme (par ex. agrégées ou calculées sur la base de données brutes). Bien que les actions, activités et objectifs (et les preuves à l'appui, sous la forme d'indicateurs ou de cibles) forment le socle du témoignage, ils ne doivent pas nécessairement être tous présentés au même niveau de détails. Seuls ceux qui ont le plus grand impact réformateur, ou qui donnent lieu aux problèmes ou lacunes les plus aigus méritent une analyse approfondie. En plus de ce récit ciblé, un résumé ou une description synthétique du progrès de chaque activité doit être fourni lorsque cela est pertinent pour le cycle de reporting. Par exemple, au lieu de rendre compte de la mise en œuvre de toutes les activités énumérées pour un objectif donné, il est recommandé de sélectionner celles qui ont le plus d'impact et de fournir un tableau général des performances positives ou négatives par rapport à l'objectif. 
L'Annexe 5 présente une structure de rapport de stratégie générale de RAP et des instructions pour l'élaboration de celui-ci, ainsi que des exemples.

\subsection{Organisation et rôles institutionnels pour le suivi et l'élaboration de rapports de stratégie}

Étant donné la nature intersectorielle et horizontale de la RAP, et puisque des institutions multiples prennent habituellement part à la mise en œuvre de la réforme, le suivi et le reporting pour une RAP représenteront probablement un processus plus complexe que pour une stratégie sectorielle. Dans les deux cas, cependant, la bonne attribution des responsabilités de mise en œuvre et de suivi est d'une importance cruciale. Ces rôles, ainsi que les méthodes de travail (le processus de suivi et de reporting), doivent être définis conjointement à l'adoption de la (ou des) stratégie(s) respective(s).

Les principales institutions impliquées dans le suivi et l'élaboration de rapports pour une stratégie de RAP sont les suivantes :

1. Le gouvernement

2. Tout conseil de la RAP ou organisme de niveau politique équivalent

3. Tout groupe interministériel sur la RAP ou autre organisme de niveau administratif équivalent

4. Les institutions chefs de file de la RAP (le ministère ou organe gouvernemental responsable)

5. Les ministères et agences gouvernementales chargés de la mise en œuvre

L'Encadré 19 présente un bref résumé de chaque niveau mentionné ci-dessus en lien avec le suivi et l'établissement de rapports pour la RAP, tandis que l'Annexe $\mathbf{5}$ fournit une description plus détaillée de ces missions.

\section{Encadré 19. Bref aperçu des fonctions de suivi et de reporting pour la RAP}

Le gouvernement est l'organe décisionnel final pour la RAP (comme pour toute politique ou secteur). Il peut orienter les efforts ou donner davantage de pouvoir à l'institution chef de file et aux autres institutions participantes pendant les réformes. Il peut régler les litiges entre différentes institutions et adopter toute mesure et solution nécessaire pour améliorer la stratégie de RAP et garantir sa mise en œuvre efficace.

Le conseil de la RAP (s'il existe) est un organisme politique décisionnaire de haut niveau qui pilote la mise en œuvre de la stratégie de RAP et fournit des avis à l'institution chef de file et aux autres institutions qui en sont chargées.

Le groupe interministériel (ou interinstitutionnel) sur la RAP (s'il existe) consiste habituellement en un ou plusieurs organismes de niveau administratif constitués de hauts fonctionnaires provenant des principales institutions impliquées. Sa mission est de s'assurer qu'il existe un suivi régulier de la mise en œuvre des objectifs, cibles, activités et budgets de la stratégie de RAP, et de résoudre tout différend de nature administrative (et non politique).

L'institution chef de file (ministère) est chargée du fonctionnement général et de la réforme de l'administration publique, et coordonne la réalisation des objectifs, indicateurs et activités de la réforme stratégique au quotidien. C'est l'institution chargée de coordonner la mise en œuvre de la stratégie de RAP au niveau de l'expertise technique. En générale, l'institution principale a pour mission de recueillir les informations et d'élaborer les rapports de suivi, 
ainsi que d'assurer le fonctionnement efficace de l'ensemble du système de suivi (et notamment de garantir la disponibilité des données et leur fourniture dans les délais).

Les ministères (et autres organes gouvernementaux) sont chargés d'assurer la mise en œuvre des activités et objectifs qui relèvent de leurs attributions (ou de contribuer à la mise en œuvre de certaines activités conformément au rôle qui leur a, le cas échéant, été attribué), ainsi que de fournir les informations nécessaires à l'institution chef de file pour effectuer le suivi de la mise en œuvre de la stratégie de RAP.

\section{Réflexions pratiques :}

$\checkmark$ Mettez toutes les lois et réglementations de côté lors de la rédaction du rapport. Tâchez de vous mettre à la place des décideurs : qu'est-ce qui les intéresse le plus, et sous quelle forme souhaiteraient-ils en être informés?

$\checkmark \quad N$ 'écrivez pas de longs rapports - c'est la qualité, et non la quantité, qui compte.

$\checkmark$ Il peut être souhaitable de consulter des collègues ou experts en communication et relations publiques afin d'avoir des idées innovantes sur la manière de présenter les principales informations de façon plus conviviale. Ils peuvent donner des idées et des conseils intéressants.

$\checkmark \quad$ La qualité rédactionnelle des rapports augmente avec la pratique. Ne soyez pas découragé si les premiers essais ne sont pas bien reçus et si les premiers rapports sont trop arides.

$\checkmark$ Analysez toujours, ne vous contentez pas de présenter!

$\checkmark \quad$ N'oubliez pas : le rapport est le meilleur outil pour influencer le changement et pour assurer le succès de la réforme.

\section{Ne pas oublier :}

$\checkmark \quad$ Un bon système de suivi, de reporting et d'évaluation exige des indicateurs bien définis, avec leurs valeurs de référence et leurs cibles.

$\checkmark$ Un suivi et un reporting solides demandent du temps, des compétences en analyss et en communication, et une réflexion créative.

$\checkmark$ Un suivi et un reporting efficaces nécessitent l'implication active des responsables et dirigeants.

$\checkmark$ Un bon rapport est concis, bien structuré, analytique et ciblé sur les résultats.

$\checkmark$ Un rapport n'est bon que s'il aide à assurer une mise en œuvre efficace et efficiente de la réforme et appuie la prise de mesures correctives si nécessaire.

$\checkmark$ Le système de suivi, de reporting et d'évaluation (avec des rôles et responsabilités clairs, une structure de rapport bien construite, etc.) devrait être élaboré conjointement à l'adoption de la stratégie.

$\checkmark$ Si le cadre de la RAP est constitué de plusieurs stratégies, les systèmes de suivi, de reporting et d'évaluation doivent être standardisés et harmonisés. 


\section{8. Évaluation}

L'évaluation est le processus plus élaboré d'analyse du succès de la mise en œuvre d'une stratégie, elle relève ce qui a fonctionné, examine les cause des échecs, et procède en conséquence à des adaptations de l'orientation stratégique. La conception et l'exécution de la phase d'évaluation sont habituellement indépendantes du cadre régulier de suivi et de reporting. L'évaluation implique d'élaborer des questions d'évaluation, de recueillir et d'analyser des données pour obtenir des réponses à ces questions, et de rassembler des éléments permettant de formuler des conclusions et recommandations. L'objectif de l'évaluation est d'améliorer la mise en œuvre ou la conception de la politique stratégique $^{45}$. Une bonne évaluation est également un outil essentiel de responsabilisation et favorise également le renforcement institutionnel ${ }^{46}$.

Comme le suivi et le reporting, l'évaluation exige des compétences spécifiques :

- des compétences méthodologiques pour structurer le cadre d'évaluation (principalement définir les questions d'évaluation);

- des compétences analytiques pour être capable de gérer la collecte et l'analyse des données ;

- l'aptitude à traduire des informations techniques en recommandations pratiques ;

- une grande facilité à travailler en réseau, car le recueil des données impliquera de contacter de nombreuses parties prenantes ;

- des compétences en présentation et en communication pour expliquer des questions techniques et complexes en langage simple, en évitant le jargon.

\subsection{Qui, et que, faut-il évaluer ?}

L'évaluation demande beaucoup plus de ressources et de compétences que le suivi. Elle requiert des compétences analytiques et une expertise plus sophistiquées dans plusieurs méthodes de recueil et d'analyse des données. Il est courant, pendant l'évaluation, d'utiliser des méthodes d'analyse statistique pour lesquelles il est possible que n'existent pas des compétences en interne au sein de l'administration (dans la principale institution/ministère). Il existe, cependant, plusieurs options pour effectuer l'évaluation - en interne avec le personnel dédié au programme, en mandatant des fournisseurs externes, ou en mélangeant approches internes et externalisées. L'Encadré 20 évalue chacune de ces options.

\footnotetext{
${ }^{45}$ Pour plus d'information sur l'évaluation, voir : Commission européenne (2017),

Better regulation guidelines - Evaluation and fitness checks, Bruxelles, https://ec.europa.eu/info/sites/info/files/better-regulation-guidelines-evaluation-fitnesschecks.pdf

Banque mondiale,

https://openknowledge.worldbank.org/bitstream/handle/10986/2699/52678.pdf, et OCDE (2002), L'évaluation et l'efficacité de l'aide $n^{\circ} 6$ - Glossaire de principaux termes relatifs à l'évaluation et la gestion axée sur les résultats (en anglais, français et espagnol), ÉDITIONS OCDE, Paris, https://doi.org/10.1787/9789264034921-en-fr.
}

${ }^{46}$ Pour une liste de principes et de questions directrices pour l'évaluation de performance, voir : OCDE (2014), «Évaluation des performances », dans La gouvernance des régulateurs, Éditions OCDE, Paris, https://doi.org/10.1787/9789264222649-fr. 


\section{Encadré 20. Avantages et limites des choix d'organisation de l'évaluation}

\begin{tabular}{|l|l|}
\hline $\begin{array}{l}\text { OPTION } 1 \text { : évaluation en } \\
\text { interne }\end{array}$ & $\begin{array}{l}\text { L'évaluation est conçue et menée en interne par le personnel } \\
\text { de l'institution chef de file. }\end{array}$ \\
Avantages : permet une évaluation plus rapide, car il n'y a \\
pas besoin de procédures d'appel d'offres, souvent \\
$\begin{array}{l}\text { longues; disponibilité d'informations en interne ; } \\
\text { renforcement des compétences et de la réflexion } \\
\text { analytiques. }\end{array}$
\end{tabular}

Limites : peut être moins objective, à cause de la réticence à révéler les défis et problèmes.

Exemples : Évaluations de moyen terme de la Stratégie de RAP serbe ${ }^{47}$.

\section{OPTION 2 : évaluation} externalisée
L'évaluation est menée par des consultants externes, par une procédure externalisée.

Avantages : évaluation objective par des évaluateurs externes et indépendants.

Limites : exige des ressources additionnelles pour financer l'expertise ; trouver les prestataires peut nécessiter un délai supplémentaire.

Exemples : Évaluation du Fonds de réforme de l'administration publique en Bosnie-Herzégovine ${ }^{48}$.

Seules certaines parties de l'évaluation sont externalisées (par ex. le recueil de données et les volets les plus complexes de l'analyse), tandis que le reste est fait en interne.

Avantages : évaluation objective grâce à la participation objective d'évaluateurs externes et indépendants ; processus d'évaluation plus rapide, car les phases plus chronophages peuvent être externalisées.

Limites : exige des ressources additionnelles pour financer certaines prestations.

Exemples : Rapport complet sur la mise en œuvre de la Stratégie de RAP du Kosovo 2010-2013 ${ }^{49}$.

Le choix de mener une évaluation en interne, externalisée ou mixte dépend également des objectifs du processus d'évaluation :

${ }^{47}$ Rapport triennal 2015-2017 sur la mise en œuvre de la stratégie de RAP et de son plan d'action, (PAR Strategy and its Action Plan), mars 2018, http://www.mduls.gov.rs/doc/PAR\%20Report_eng_mar2018.pdf ). 
- Autoréflexion et apprentissage organisationnel - une évaluation en interne est plus appropriée car elle permet une meilleure réflexion en interne.

- Déterminer quelle option politique a le meilleur rapport coût-efficacité - une évaluation externalisée est plus adaptée car elle utilise des méthodes d'évaluation plus sophistiquées, et livre des avis plus objectifs et indépendants.

- Vérifier que les activités ont été menées et les résultats atteints comme prévuune évaluation externalisée est plus appropriée car une telle vérification peut exiger des avis plus objectifs et indépendants ${ }^{50}$.

Dans tous les cas, il est nécessaire de prendre en compte les coûts ainsi que les compétences. Les pays mènent souvent des évaluations externalisées en raison d'un manque de compétences et d'aptitudes en interne. Il faut du temps et des ressources pour développer les compétences d'évaluation requises. D'un autre côté, il existe un besoin croissant d'évaluations rapides, pour analyser des défis et problèmes apparus inopinément et auxquels il faut apporter des solutions intermédiaires. De telles évaluations sont menées en interne et emploient des outils et méthodes de recueil et d'analyse des données moins sophistiqués.

L'évaluation devrait être sélective et ciblée. Il n'est pas nécessaire (et pas non plus recommandé) de mener une évaluation chaque année. Les décisions sur les aspects à évaluer dépendent souvent des données fournies par les processus de suivi. Par exemple, il peut être utile d'entreprendre une évaluation quand les données de suivi montrent que certaines cibles n'ont jamais été atteintes, ou si les usagers des services se sont plaints de leur qualité. Les mesures dotées de gros budgets ou concernant des questions importantes pour le programme politiques (les priorités) sont probablement celles qui se prêtent le mieux à l'évaluation.

Globalement, une évaluation doit mener à des décisions, et encourager les administrations à :

- Débuter, arrêter ou continuer une stratégie nouvelle ou existante ;

- réviser la politique - les objectifs, actions, activités, ou les indicateurs et leurs cibles ;

- réviser les budgets et les ressources ;

- ajuster les processus de mise en œuvre - calendriers, responsabilités ;

- tirer les leçons des succès, des échecs et de leurs causes.

\subsection{Type et portée de l'évaluation}

L'évaluation d'une stratégie peut être intermédiaire (effectuée au cours de la mise en œuvre de la stratégie) ou ex-post (effectuée une fois la stratégie mise en oeuvre). Les

48 SIDA Decentralised Evaluation: Evaluation of the Public Administration Reform Fund in Bosnia and Herzegovina, avril 2015, https://www.sida.se/contentassets/7f6661a1ee4046bfa45e4295d26b1c5e/15538.pdf.

49 Comprehensive Report on Implementation of The Public Administration Reform Strategy 2010-2013, juin 2014, https://map.rks-gov.net/getattachment/8548fd1f-60d2-4318-b2268f1ed8e26d27/Raporti-gjithperfshires-mbi-zbatimin-Strategjise-d.aspx.

${ }^{50}$ Pour plus d'informations sur les bénéfices potentiels de l'évaluation, voir : OCDE (2009), "Evaluation Improves Performance", dans Focus on Citizens: Public Engagement for Better Policy and Services, Éditions OCDE, Paris, https://doi.org/10.1787/9789264048874-6-en. 
évaluations sont classées selon qu'elles sont axées sur le processus, le programme ou l'impact. Le Tableau 5 présente une brève description de chaque type d'évaluation.

\section{Tableau 5. Types d'évaluation}

\begin{tabular}{|c|c|c|c|}
\hline & Évaluation de processus & $\begin{array}{l}\text { Évaluation de } \\
\text { programme }\end{array}$ & Évaluation d'impact \\
\hline Axe & $\begin{array}{l}\text { Efficience, mise en œuvre, } \\
\text { mécanismes internes, mise } \\
\text { en œuvre/exécution des } \\
\text { réalisations }\end{array}$ & $\begin{array}{l}\text { Efficacité, apport, } \\
\text { obtention de résultats }\end{array}$ & Cause à effet, portée, impact \\
\hline Méthodes & $\begin{array}{l}\text { Analyse des tâches, } \\
\text { comparaison des activités } \\
\text { menées et des réalisations } \\
\text { obtenues avec celles qui } \\
\text { étaient prévues }\end{array}$ & $\begin{array}{l}\text { Analyse comparative, } \\
\text { analyse } \\
\text { rentabilité/bénéfice, } \\
\text { benchmarking }\end{array}$ & $\begin{array}{l}\text { Analyse contrefactuelle, groupes de } \\
\text { comparaison, analyse statistique, } \\
\text { randomisation }\end{array}$ \\
\hline $\begin{array}{l}\text { Question à } \\
\text { laquelle il } \\
\text { est répondu }\end{array}$ & $\begin{array}{l}\text { La mise en œuvre s'est-elle } \\
\text { déroulée comme prévu, et } \\
\text { les résultats semblent-ils } \\
\text { accessibles? }\end{array}$ & $\begin{array}{l}\text { La politique a-t-elle été } \\
\text { efficace pour atteindre les } \\
\text { objectifs fixés? }\end{array}$ & $\begin{array}{l}\text { Que se serait-il passé en l'absence } \\
\text { de la politique/de la réforme? }\end{array}$ \\
\hline
\end{tabular}

La portée de l'évaluation est une autre question sur laquelle les responsables et les équipes doivent se prononcer à l'avance. elle est déterminée en appliquant cinq critères d'évaluation (voir Tableau 6) et en sélectionnant les plus pertinents selon le moment où à lieu l'évaluation, sa durée, les besoins et les ressources disponibles ${ }^{51}$. Ces critères et questions aident à définir la structure et la portée de l'évaluation. Il n'est pas nécessaire de tout demander en même temps. Pour sélectionner les points à évaluer, on peut se fonder sur les raisons pour lesquelles une évaluation a été décidée; et de qui en est attendu ${ }^{52}$.

${ }^{51}$ Il faut noter que, selon les Lignes directrices de l'UE pour une meilleure réglementation, les critères pertinents sont : efficacité, efficience, pertinence, cohérence et valeur ajoutée de l'UE comme critères obligatoires, tandis que l'impact, la durabilité, l'utilité, la complémentarité, la coordination, l'équité, l'acceptabilité sont des critères additionnels pour l'évaluation. Pour plus d'informations, voir Commission européenne, Better Regulation Guidelines, Bruxelles, p. 52.

52 Pour plus d'informations sur les critères d'évaluation, voir : http://www.oecd.org/fr/cad/evaluation/criteres-cad-evaluation.htm. 
Tableau 6. Critères et questions d'évaluation

\begin{tabular}{|c|c|}
\hline Critères & Questions \\
\hline Pertinence & 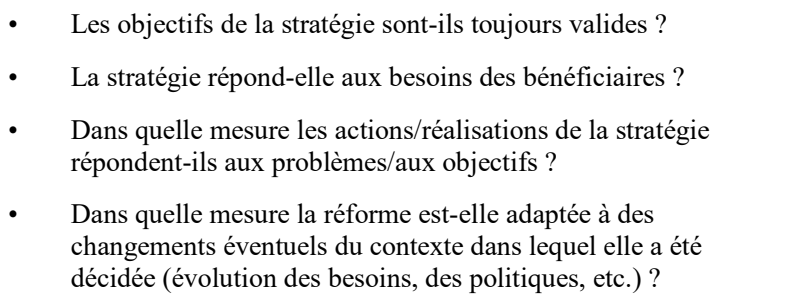 \\
\hline Efficience & $\begin{array}{l}\text { - L'action/l'activité est-elle efficiente en termes de coût? } \\
\text { - } \quad \begin{array}{l}\text { Dans quelle mesure les coûts induits sont-ils justifiés, au vu des } \\
\text { changements/des effets qui ont été atteints? }\end{array} \\
\text { - } \quad \text { Quelles sont les autres utilisations des ressources qui ont produit } \\
\text { plus de résultats, ou qui ont été plus économiques tout en } \\
\text { maintenant le même niveau de résultats? } \\
\text { - Les mêmes résultats auraient-ils pu être obtenus en utilisant } \\
\text { moins de ressources, ou par une combinaison } \\
\text { meilleure/différente des ressources utilisées? } \\
\text { Quel est le degré d'efficience (de fluidité) du processus de mise } \\
\text { en œuvre? }\end{array}$ \\
\hline Efficacité & $\begin{array}{l}\text { Dans quelle mesure les objectifs ont-ils été atteints/sont-ils en } \\
\text { voie d'être atteints ? } \\
\text { - Les objectifs ont-ils été atteints à temps ou le seront-ils? } \\
\text { - Dans quelle mesure les effets observés correspondent-ils aux } \\
\text { objectifs? } \\
\text { Quelles sont les principales raisons pour lesquelles les objectifs } \\
\text { sont ou ne sont pas atteints? }\end{array}$ \\
\hline Impact & $\begin{array}{l}\text { - Que s'est-il passé du fait de la stratégie ? } \\
\text { Quelle différence la stratégie a-t-elle apportée aux différents } \\
\text { groupes de bénéficiaires (et au public dans son ensemble)? } \\
\text { - Comment les différents groupes cibles ont-ils été affectés? }\end{array}$ \\
\hline Durabilité & $\begin{array}{l}\text { - Les bénéfices continueront-ils après la mise en œuvre de la } \\
\text { stratégie ? } \\
\text { - } \quad \text { Les impacts peuvent-ils être considérés comme durables ? } \\
\text { - Comment s'assurer que les impacts perdurent? } \\
\text { - Quelles dispositions institutionnelles permettraient aux résultats } \\
\text { favorables acquis de perdurer? }\end{array}$ \\
\hline
\end{tabular}

Puisqu'une évaluation demande habituellement du temps et des efforts, il est important de la programmer. Si la mise en œuvre d'une stratégie est envisagée en plusieurs étapes (traduites dans des plans d'actions consécutifs), il est conseillé de prévoir le processus d'évaluation ainsi que la conception du cadre de suivi, de reporting et d'évaluation en même temps qu'est adoptée la stratégie. Ceci permettra d'utiliser les résultats d'une évaluation de moyen terme pour élaborer un nouveau plan d'action, et même réorienter 
en cours d'application le cadre stratégique global (vérifier la justesse des objectifs et cibles d'indicateurs choisis au départ, ainsi que le succès de la mise en œuvre). Par ailleurs, l'évaluation représente un coût en soi et doit être prise en compte dès le début. L'Annexe 5 présente un exemple de cahier des charges pour l'évaluation externe.

\section{Réflexions pratiques :}

$\checkmark$ La clé d'une évaluation réussie réside dans une planification rigoureuse du processus d'évaluation soigneusement planifié, avec une sélection des évaluateurs, un recensement des données nécessaires pour répondre à ces questions identifiées, les méthodes d'analyse étant choisies à l'avance. L'estimation des ressources nécessaires et leur affectation a de même été faite.

$\checkmark$ Étant donné que la participation aux évaluations représente une bonne manière d'apprendre, il est toujours avantageux d'envisager d'impliquer les personnels clé dans le processus d'évaluation (au moins partiellement).

$\checkmark \quad$ L'évaluation n'est utile que s'il existe, chez les différents décideurs et le public plus large des parties prenantes, une volonté d'apprendre et de s'adapter.

Ne pas oublier :

$\checkmark$ L'évaluation n'est effectuée qu'occasionnellement. L'évaluation doit être un événement spécial!

$\checkmark$ L'évaluation peut être effectuée en interne, être externalisée ou une combinaison des deux.

$\checkmark$ L'évaluation doit être sélective et n'analyser que les questions les plus problématiques.

$\checkmark \quad$ L'évaluation requiert une expertise et des compétences spécifiques, qui peuvent ne pas être immédiatement disponibles en interne.

$\checkmark \quad$ L'évaluation se fonde sur les données de suivi, mais son objectif est différent et plus stratégique.

$\checkmark$ L'évaluation doit être programmée. 


\section{Gestion et coordination de la RAP}

Étant donné la nature transversale de la RAP et les institutions multiples généralement impliquées dans la mise en œuvre des réformes, il est conseillé d'avoir des structures de pilotage et de coordination dédiées pour superviser la mise en œuvre de la RAP. De fait, cette pratique existe déjà dans plusieurs pays candidats et potentiellement candidats à l'UE, ainsi que dans certains pays de la PEV. Alors qu'il est d'une importance capitale de créer de telles structures afin d'assurer la réussite de la mise en œuvre, celles-ci ont souvent déjà été créées par les pays pour l'élaboration de leurs stratégies de RAP. Dans tous les cas, la création des structures, les institutions (et souvent les individus nommés), leurs rôles et responsabilités, et les procédures de travail des structures devraient être formalisées dans une décision séparée ou expliquées dans les documents stratégiques de la RAP. Étant donné que la RAP ne constitue pas un domaine politique autonome et n'existe pas isolément, quelle que soit la structure de gestion créée pour l'élaboration et la mise en œuvre d'une stratégie de RAP, elle doit être conforme aux modalités et aux processus suivies par le pays lors de l'élaboration et de la coordination des politiques.

Les structures peuvent être créées au niveau politique (par exemple sous la forme d'un conseil ou comité ministériel de la RAP) et au niveau administratif (comme par des groupes de travail interministériels ou des groupes de travail sectoriels). En règle générale, il est souhaitable d'avoir une structure de coordination au niveau politique et au niveau administratif, pour garantir une gestion de la réforme ciblée et efficace. Comme conseils a minima, l'Encadré 21 présente les éléments clés des missions respectives (rôles et responsabilités) des structures de niveau politique et de niveau administratif.

\section{Encadré 21. Missions des structures de coordination de la RAP}

STRUCTURE MISSION

Structure de niveau 1. Piloter et superviser la mise en œuvre de la RAP et de toutes les politique : $\quad$ stratégies contenues dans le cadre stratégique de la RAP. Prendre ou proposer des mesures correctives pour garantir la mise en œuvre efficace des réformes. L'examen, par des rapports de stratégie de RAP semestriels, annuels et ad hoc, doit inclure un contrôle régulier de la mise en œuvre des objectifs et activités, de l'atteinte des objectifs, de l'exécution du budget, des déficits de financement, etc.

2. Débattre, et fournir des avis stratégiques, sur les enjeux particuliers liées à la RAP, notamment les orientations politiques précises. Examiner et débattre des projets de décisions liées à la RAP qui sont présentées au gouvernement pour approbation (telles que projets de loi, réglementations, documents conceptuels, etc.)

3. Coordonner et harmoniser les opinions divergentes (résolution des litiges). Examiner d'autres questions liées à la RAP et résoudre 
tout défi qui émerge pendant la mise en œuvre de la stratégie de RAP.

4. Organiser la communication sur l'élaboration et la mise en œuvre de la réforme à l'intérieur et à l'extérieur de l'administration.

Structure de niveau administratif :
1. Identifier les lacunes dans la mise en œuvre de la stratégie de RAP et les opinions divergentes sur les questions liées à la RAP. Superviser et examiner les progrès dans la mise en œuvre des activités des stratégies de RAP, sur une base ad hoc ou par des rapports réguliers.

2. Accompagner le travail effectif de la direction politique et les discussions du conseil de la RAP (ou son équivalent) en préparant les documents pour les délibérations politiques (rapports, notes de politique, etc.) et en favorisant la communication et la visibilité. Examiner les projets de décisions liées à la RAP qui sont présentées au conseil de la RAP (telles que projets de loi, réglementations, documents conceptuels, etc.)

3. Coordonner les actions des institutions chargées de mettre en œuvre les diverses activités liées à la RAP, et examiner et résoudre les problèmes qui entravent ou gênent la mise en œuvre réussie et dans les délais des activités, objectifs et indicateurs de la RAP.

4. Sur la base des informations analytiques et opérationnelles dont dispose l'organe interministériel, examiner les modifications nécessaires de la stratégie de RAP et les proposer à l'institution chef de file ou au conseil de la RAP.

\subsection{Composition des structures de gestion}

\subsubsection{La structure de niveau politique}

L'organe de niveau politique est habituellement constitué de membres du gouvernement du pays (ou au moins de leurs adjoints politiques). Si la RAP représente une priorité du gouvernement, cet organe peut être présidé par le chef du gouvernement (le Premier ministre) ou au moins par le chef de l'institution (le ministre) chargée de l'administration publique. La présence du ministre des finances et, dans le cas des pays candidats ou potentiellement candidats à l'adhésion à l'UE, du ministre en charge de l'intégration européenne, constitue également une bonne pratique. D'autres chefs d'institutions (ministres) sont choisis et nommés selon leur rôle dans le processus de réforme. Si les réformes concernent également des institutions indépendantes du gouvernement (par exemple le système judiciaire), leurs dirigeants politiques devraient aussi être membres de l'organe de niveau politique. De plus, il est conseillé d'impliquer des parties prenantes externes clés (comme des représentants d'ONG ou du monde universitaire) dans l'organe de niveau politique, en tant que membres ou comme observateurs, pour renforcer la responsabilité et la crédibilité de l'engagement du gouvernement envers la réforme.

Le rôle de la structure politique (par ex. le conseil de la RAP) dans le processus de suivi et de reporting est crucial, car elle possède tous les outils pour fournir des retours 
de haut niveau et des incitations aux institutions participantes à s'efforcer d'atteindre les objectifs fixés. Sur la base de l'information contenue dans les rapports, les décideurs de haut niveau peuvent utiliser leur poids politique pour " pousser» les institutions à aller plus vite pour atteindre les objectifs ou, si les données montrent que cela n'est pas possible, pour réviser la stratégie. Il est recommandé que le conseil de la RAP se réunisse au moins tous les six mois afin de débattre dans un délai raisonnable des progrès de la mise en œuvre et des difficultés qui ont pu se manifester. Les réunions du conseil de la RAP sont décidées par son président et organisées par le secrétariat du conseil de la RAP. Le secrétariat du conseil de la RAP est, en règle générale, tenu par l'institution chef de file de la RAP (le ministère).

\subsubsection{La structure de niveau administratif}

Outre l'organe de niveau politique, un organe administratif (ou interministériel) ou plusieurs groupes de travail thématiques devraient être créés pour coordonner les différents domaines de la RAP à un niveau plus technique. Ces organes interministériels sont composés des représentants des principales institutions (habituellement les ministères et agences) qui mettent en œuvre les domaines couverts par la stratégie de RAP. Le président de la structure est habituellement un haut fonctionnaire issu de l'institution chef de file (ministère) chargée de la RAP. Si des organes de niveau administratif spécifiques par domaines sont créés, ils devraient être dirigés par l'institution qui supervise le domaine en question lié à la RAP. La principale mission de tels groupes de travail thématiques est similaire à celle d'un groupe interministériel unique, mais limitée à leur domaine de RAP respectif. Les membres des organes de niveau administratif devraient être des fonctionnaires compétents (de préférence de niveau cadre, avec un certain pouvoir décisionnel). Des parties prenantes externes devraient également être invitées aux réunions de la (des) structure(s) de niveau administratif, soit au cas par cas, soit de façon permanente (prévue lors de la création et de la formalisation des structures).

Il est recommandé que l'organe interministériel se réunisse au moins tous les trois mois (trimestriellement) pour débattre des progrès liés à la mise en œuvre des activités. Les réunions de l'organe interministériel sont décidées par son président et organisées par le secrétariat (à savoir l'institution chef de file de la RAP)

\subsubsection{Le secrétariat}

Il est également conseillé de désigner un secrétariat pour servir de soutien aux structures de niveau politique et de niveau administratif. Les principales fonctions du secrétariat sont de :

- Contribuer à la préparation des réunions du conseil de la RAP et de l'organe interministériel en élaborant leurs programmes, en gérant les invitations et la distribution des documents, et en préparant et diffusant les minutes et les décisions après leurs réunions respectives ;

- Coordonner la préparation des documents de travail pour les réunions et examiner leur qualité ;

- Coordonner l'élaboration de rapports de suivi réguliers et de toute tâche analytique associée au suivi et à l'évaluation ;

- Coordonner et soutenir les activités de communication et de visibilité associées au processus de réforme ;

- Développer et gérer l'utilisation du système de suivi de la réforme, en diffusant des lignes directrices et en dispensant des conseils et de la formation ; 
- Garantir que la mise en œuvre de la stratégie adoptée et que toute décision intérimaire prise en cours d'exécution par les forums de niveau politique ou administratif sont correctement respectées et prises en compte ;

- Lorsqu'il existe plusieurs stratégies liées à la RAP, coordonner les questions à débattre et les documents à présenter avec les diverses institutions respectives chargées des différentes stratégies liées à la RAP.

Du point de vue organisationnel, l'institution chef de file de la RAP doit être capable de :

- Proposer, bien à l'avance des réunions, des points d'ordre du jour pertinents et bien préparés ;

- préparer des notes et des propositions de décisions politiques ;

- sélectionner sur leurs compétences des participants non permanents aux réunions, comme des représentants d'ONG, du milieu universitaire, d'associations et de professions (lorsque ceux-ci n'ont pas déjà un statut de membres prédéterminé).

Lorsqu'un pays a plusieurs stratégies liées à la RAP (y compris des stratégies de GFP), il est recommandé d'utiliser une structure unique de gestion de niveau politique - un conseil de la RAP unique, par exemple - pour rationaliser les flux d'informations et de décisions, notamment celles qui concernent l'attribution des ressources. Dans ce cas, l'institution chef de file de la RAP devra coordonner la préparation des documents (rapports réguliers et documents décisionnels liés à la RAP) et les débats avec les autres institutions responsables des différents piliers des réformes, comme le ministère des Finances et le ministère chargé de la réforme de la fonction publique. Les structures de niveau administratif peuvent demeurer "décentralisées », c'est-à-dire que chaque stratégie différente liée à la RAP peut avoir sa propre structure de niveau administratif.

La gestion des finances publiques (GFP) est un domaine clé de la RAP. Pourtant, la coordination et la gestion des réformes de GFP ainsi que la mise en œuvre des stratégies correspondantes relèvent souvent de structures de gestion et de coordination séparées. Si ce n'est pas un problème en soi, assurer l'harmonisation parfaite des méthodes de travail, des modalités d'élaboration et de mise en œuvre de la stratégie, des moyens et des liaisons entre les résultats de l'activité de telles structures séparées de gestion et de coordination exige d'avantage de rigueur et ce doit toujours être le cas. Cet indispensable pour que puissent être constatés les progrès des différents aspects de la RAP, y compris la GFP qui y est intimement liée. Si des structures séparées sont créées pour la GFP et d'autres domaines de la RAP, il est conseillé que les institutions chef de file sur différents sujets soient membres des autres structures de gestion thématiques (au niveau administratif comme au niveau politique, bien que pour ce dernier, il est souhaitable de n'avoir qu'une seule structure) et qu'elles échangent régulièrement des opinions et des informations. Outre l'étroite coopération entre institutions chef de file, il est fortement conseillé de rationaliser les processus de travail et les outils utilisés pour la coordination et la gestion des réformes, notamment d'utiliser le même cadre, ou un cadre similaire de suivi et de reporting, ainsi que de prévoir les activités de coordination de manière harmonisée (par ex. établir des rapports à la même fréquence).

\subsection{Facteurs de réussite dans la gestion de la RAP}

L'efficacité des organes de gestion de niveau politique et administratif dépend fortement, outre des moyens humains et financiers appuyant la mise en œuvre et, plus spécifiquement, la gestion de la RAP, de la qualité des documents qu'ils examinent et 
grâce auxquels ils prennent des décisions. L'utilisation ou non du poids potentiel de l'organe de niveau politique dépend de nombreux facteurs, notamment :

- De la compréhension par les décideurs de l'importance du programme de RAP ;

- du soin avec lequel les rapports et autres documents sont préparés (à savoir s'ils exposent bien les principaux défis, les lacunes et risques qui doivent être communiqués ;

- de la qualité avec laquelle les documents stratégiques sont rédigés et communiqués.

Les documents de travail pour les réunions du conseil de la RAP et de l'organe interministériel sont préparés par ceux qui doivent présenter le sujet, en concertation avec le secrétariat. Si nécessaire, le secrétariat peut demander à d'autres membres de l'organe interministériel de contribuer à la préparation de ces documents de travail. Les documents qui doivent être présentés aux réunions du conseil de la RAP ou de l'organe interministériel doivent être envoyés au secrétariat pour être distribués à tous les membres de l'organe de gestion concerné.

Le secrétariat doit toujours vérifier la qualité des documents préparés par les autres membres ou intervenants, et le rédacteur du document doit toujours réviser celui-ci, lorsqu'il y est convié, pour répondre aux critères de qualité du secrétariat.

Les documents et questions qui doivent être présentées au conseil de la RAP doivent être examinés par l'organe interministériel avant les réunions du conseil de la RAP, pour les rationaliser et ainsi réduire la charge pesant sur les décideurs au niveau politique, et s'assurer que tous les litiges au niveau administratif sont résolus au bon niveau de management. En cas d'urgence, les membres de l'organe interministériel peuvent être consultés sur certaines questions par voie électronique (emails), uniquement avant la réunion du conseil de la RAP.

Le secrétariat doit préparer un projet de décision pour chaque question à l'ordre du jour du conseil de la RAP ou de l'organe interministériel, et le soumettre avec les documents de la réunion. La décision doit être ultérieurement amendée par le secrétariat, conformément aux résultats de la discussion lors de la réunion, et la décision finale doit ensuite être diffusée à tous les membres de l'organe concerné.

La réussite des réformes dépend aussi d'une discussion approfondie sur les questions politiques dans le conseil de la RAP (ou équivalent) et la(les) structure(s) administrative(s). C'est pourquoi il est nécessaire que l'institution chef de file de la RAP, qui remplit habituellement le rôle de secrétariat de la RAP, ait les compétences nécessaires pour contribuer à une prise de décisions politiques pleinement informée et reposant sur des éléments avérés. Disposer de documents de politique stratégique bien conçus permet au conseil de la RAP de réfléchir aux questions liées à la RAP de façon plus ciblées et plus approfondie, qu'il n'est en général possible de le faire lors des sessions gouvernementales, au cours desquelles il y a beaucoup plus de questions nécessitant une décision que pendant une réunion aux sujets bien définis et spécifiques du conseil de la RAP. L'Encadré 22 présente un exemple de document stratégique exhaustif et bien ciblé permettant la prise de décision. 


\section{Encadré 22. Court exemple théorique d'un document à fin de décision}

Conseil de la RAP

Date de la réunion : $1^{\text {er }}$ octobre 2017

Lieu : Immeuble gouvernemental

MINUTES DE LA RÉUNION DU CONSEIL DE LA RAP

\section{PARTICIPANTS :}

Membres du Conseil de la RAP : Premier ministre (Président), ministre de l'Administration publique (Vice-président), ministre des Finances, ministre des Transports et des Communications, ministre de l'Intérieur, Directeur de l'Agence de la fonction publique, Directeur de l'Agence pour l'administration électronique. Absent: ministre de l'Administration régionale et territoriale.

Autres : Secrétariat du Conseil de la RAP, Département du budget du ministère des Finances, Département pour une meilleure régulation économique du ministère des Finances.

\section{POINTS À L'ORDRE DU JOUR EXAMINÉS :}

1) Rapport de progrès annuel de la RAP

2) Système de mesure de la satisfaction des usagers

\section{CONCLUSIONS ET DÉCISIONS :}

\section{1) Rapport de progrès annuel de la RAP}

Le Conseil de la RAP, après avoir entendu les informations présentées par le Secrétariat de la RAP sur les progrès réalisés par rapport aux objectifs de la stratégie de RAP, et les contributions des autres institutions engagés dans sa mise en œuvre, est parvenu aux conclusions suivantes :

a) L'atteinte des objectifs et la réalisation des activités en matière de coordination et de responsabilisation des politiques est globalement sur la bonne voie.

b) Des retards importants affectent l'atteinte des objectifs en matière de réforme de la fonction publique et de l'administration électronique, ce qui nécessite des efforts supplémentaires pour mener à bien les travaux des institutions concernées. En particulier, des efforts communs des différentes institutions devront être consentis pour finaliser le Document conceptuel sur la gestion de la haute fonction publique. Il faudra en outre accélérer l'acquisition des services nécessaires à la création de la plateforme d'interopérabilité, afin de garantir son achèvement dans les délais.

c) L'implication de parties prenantes externes dans l'élaboration de nouvelles politiques et de nouvelles lois n'est ni suffisante ni assez rapide. L'achèvement des activités prévues subit donc des retards, la qualité de leur mise en œuvre est menacée, et des propositions politiques sont soumises au Conseil de la RAP sans concertation adéquate.

Le Conseil de la RAP attribue les principales tâches suivantes aux institutions suivantes :

d) L'Agence de la fonction publique, en partenariat avec le ministère des Finances, devra finaliser le Document conceptuel sur la gestion de la haute fonction publique avant le 31 décembre 2017. Le Document conceptuel devra entre autres comprendre l'option d'un modèle totalement centralisé de recrutement et de sélection des hauts 
fonctionnaires. L'Agence de la fonction publique devra consulter les institutions pertinentes et les représentantes des principales organisations de la société civile.

e) Le ministère des Finances, en partenariat avec l'Agence de la fonction publique, devra calculer avant le 15 décembre 2017 les implications financières des différentes options pour un nouveau modèle de gestion de la haute fonction publique. Ces calculs seront inclus dans le Document conceptuel.

f) Le ministère de la Justice, en partenariat avec l'Agence de la fonction publique, devra identifier avant le 15 décembre 2017 les lois et règlements qu'il faudra reviser pour mettre efficacement en œuvre les options pour un nouveau modèle de gestion de la haute fonction publique. Ses conclusions figureront dans le Document conceptuel.

g) Le ministère des Transports et des Communications, en coopération avec l'Agence pour l'administration électronique, devra finaliser le dossier de marché public relatif à la plateforme d'interopérabilité avant le 30 novembre 2017.

h) L'Agence des marchés publics devra donner la priorité au dossier de marché public et en assurer l'évaluation de la documentation conformément aux procédures établies.

i) Toutes les institutions chargées d'activités liées à la RAP devront veiller à ce que les parties prenantes externes soient régulièrement et rapidement consultées et s'investissent pleinement. Les résultats de ces consultations seront résumés et présentés avec les propositions soumises à l'examen du Conseil de la RAP.

\section{2) Système de mesure de la satisfaction des usagers}

Le Conseil de la RAP, après avoir entendu les informations présentées par le ministère de l'Administration publique sur les progrès de la création d'un système de mesure de la satisfaction des usagers envers les services fournis par les institutions centrales, et parvenus aux conclusions suivantes :

a) L'option 2, qui prévoit de combiner enquêtes en ligne et de terrain pour mesurer le degré de satisfaction envers cinq services prioritaires, aura la plus grande portée et permettra d'obtenir les meilleurs retours des usagers dans le cadre financier prévu.

Le Conseil de la RAP attribue les tâches ci-après aux institutions suivantes :

b) Le ministère de l'Administration publique devra rédiger des amendements à la Loi sur l'administration publique ainsi que les décrets qui s'imposent pour rendre obligatoire à partir de 2019 la mesure de la satisfaction des usagers dans certains services prioritaires.

c) Le ministère de l'Administration publique devra élaborer les méthodologies nécessaires aux enquêtes, si besoin par des procédures externalisées, avant le 30 juin 2018.

d) Les ministères et agences devront, comme il est indiqué dans le Document conceptuel, élaborer des enquêtes en ligne pour les usagers de services, à l'aide du cadre fourni dans le Document conceptuel.

e) Le ministère des Transports et des Communications, en coopération avec l'Agence pour l'administration électronique, devra créer, avant le 30 juin 2018, une installation technique permettant la mesure de mesurer en ligne la satisfaction des usagers envers les services désignés comme prioritaires. 


\section{Ne pas oublier :}

$\checkmark \quad$ La(les) structure(s) de gestion et leur mission, composition et procédures de travail doivent être établies et formalisées, que ce soit dans la stratégie de RAP ou dans une décision formelle distincte. En tout état de cause, une synthèse des principaux organes de gestion doit figurer dans la stratégie.

$\checkmark \quad$ La principale mission du conseil de la RAP est d'examiner les questions politiques liées à la RAP en se fondant sur des documents bien préparés.

$\checkmark$ Le conseil de la RAP pilote la mise en ceuvre de la stratégie de RAP et fournit des avis stratégiques sur des questions distinctes liées à la RAP, notamment sur ce qui doit être communiqué à propos du processus de réforme, quand, comment et par qui.

$\checkmark \quad$ La structure de coordination de niveau administratif peut être un organe interministériel ou plusieurs groupes thématiques. Dans ce dernier cas, un secrétariat efficace pour coordonner le travail est d'autant plus nécessaire.

$\checkmark$ Le personnel du secrétariat doit posséder de bonnes compétences de programmation, de préparation de documents (notamment de bonnes compétences en assurance qualité) et de préparation des projets de décisions du conseil de la RAP et des organes de niveau administratif. 


\section{Glossaire de certains termes clés utilisés dans le Manuel}

Action : moyens par lesquels un pays (généralement, mais pas exclusivement, le gouvernement de ce pays) met en œuvre ses politiques. Une action est une activité tangible ou un ensemble d'activités qui sont directement liées à l'utilisation d'intrants (ressources humaines, financières ou matérielles), afin de produire certains livrables prédéfinis ou d'atteindre des objectifs politiques spécifiques.

Activité : tout type de mesure, programme, projet (ou similaire) faisant référence à une série d'actions plus spécifiques devant être exécutées afin de mettre en œuvre l'action et de produire le ou les effets correspondants.

Analyse du problème : analyse de la situation existante en vue d'identifier les problèmes et leurs causes, les défis et les opportunités de réforme.

Cadrage : définition de la portée des questions de politique dans un domaine politique plus vaste (par exemple, un secteur) ou des domaines (transversaux) pour effectuer des réformes par le biais d'une stratégie basée sur les résultats d'une analyse et d'une hiérarchisation des problèmes.

Cible : indique l'ampleur et l'orientation des changements attendus.

Indicateur : facteur ou variable quantitatif ou qualitatif offrant un moyen simple et fiable de mesurer les résultats, de refléter les changements liés à une intervention ou d'aider à évaluer la performance d'un acteur. ${ }^{53}$.

Impact : effet global et à long terme de l'obtention de résultats spécifiques. Exprime l'amélioration ou le changement intervenus in fine dans la qualité de la vie et des services des individus.

Intrant : valeur ou quantité de ressources utilisées pour produire un résultat.

Objectif : un énoncé de base qui définit l'orientation des réformes en fonction des défis identifiés, de manière à éclairer l'orientation à donner aux actions de réforme et à définir de manière concrète l'état futur souhaité.

Partie prenante : une personne, un groupe ou une organisation s'intéressant à un domaine politique, une question ou des problèmes de politique spécifiques. Les parties prenantes peuvent être internes (au sein de l'administration) ou externes (organisations de la société civile, partenaires du développement, associations professionnelles, etc.).

Élément concret : tout fait, donnée ou information disponible indiquant si une croyance, un jugement ou une proposition affirmés est averé ou fondé.

Hiérarchisation : révision et sélection des problèmes à traiter, en fonction de leur urgence, de leur importance ou de leur ampleur, conformément aux priorités du gouvernement et aux ressources disponibles et à la lumière des points de vue des institutions concernées et de la communauté des parties prenantes au sens large.

Réalisation : produits ou biens et services produits par le biais d'activités ou d'actions.

Résultat : conséquences et accomplissements réalisés grâce aux effets d'une action.

${ }^{53}$ Groupe de travail du CAD sur l'évaluation de l'aide de l'OCDE, (2010a) Glossaire des principaux termes relatifs à l'évaluation et la gestion axée sur les résultats 
Stratégie : document de planification à moyen terme qui définit les ambitions de réforme dans un domaine politique plus large (par exemple, un secteur) ou des domaines (transversaux).

Valeur cible : la valeur d'un indicateur devant être atteinte à un moment donné.

Valeur de référence: une expression de la situation existante (idéalement la performance présente ou passée de l'aspect mesuré).

Vision : énoncé de l'état futur souhaité par rapport à l'état de l'administration publique en général ou dans un secteur particulier. 


\section{Le Programme SIGMA}

SIGMA (soutien à l'amélioration des institutions gouvernementales et des systèmes de gestion) est une initiative conjointe de l'OCDE et de l'Union européenne, principalement financée par l'UE. SIGMA travaille avec des pays partenaires à l'amélioration des systèmes de gouvernance et des capacités de l'administration publique depuis 25 ans.

En partenariat avec la Direction générale du voisinage et des négociations d'élargissement (DG NEAR) de la Commission européenne (CE), nous travaillons actuellement avec :

L'Albanie, la Bosnie-Herzégovine, l'Ex-République yougoslave de Macédoine, le Kosovo, le Monténégro, la Serbie et la Turquie en tant que candidats ou candidats potentiels à l'UE; et

L'Algérie, l'Arménie, l'Azerbaïdjan, l'Égypte, la Géorgie, la Jordanie, le Liban, la Moldavie, le Maroc, la Tunisie et l'Ukraine en tant que Pays du voisinage européen.

SIGMA apporte une aide dans six domaines clés :

1. Cadre stratégique de la réforme de l'administration publique

2. Élaboration et coordination des politiques

3. Fonction publique et gestion des ressources humaines

4. Responsabilité

5. Prestation de services

6. Gestion des finances publiques, passation de marchés publics et audit externe.

SIGMA examine et donne des avis sur :

- Les systèmes de gouvernance et les institutions

- Les cadres juridiques

- Les stratégies de réforme et les plans d'action

- Les progrès de mise en œuvre des réformes.

SIGMA fournit :

- Des conseils sur la conception et la priorisation des réformes

- Des méthodologies et outils pour soutenir la mise en œuvre

- Des recommandations pour améliorer les lois et les dispositions administratives

- Des opportunités pour partager les bonnes pratiques provenant de nombreux

pays, notamment au cours d'événements régionaux

- Des documents d'orientation et des études comparatives sur plusieurs pays.

Pour plus d'informations sur SIGMA, consultez notre site internet : www.sigmaweb.org

\section{(C) OCDE 2018}

SIGMA faisant partie de l'Organisation de coopération et de développement économiques (OCDE), les mêmes conditions d'utilisation s'appliquent à ses publications :

http://www.oecd.org/fr/conditionsdutilisation. 